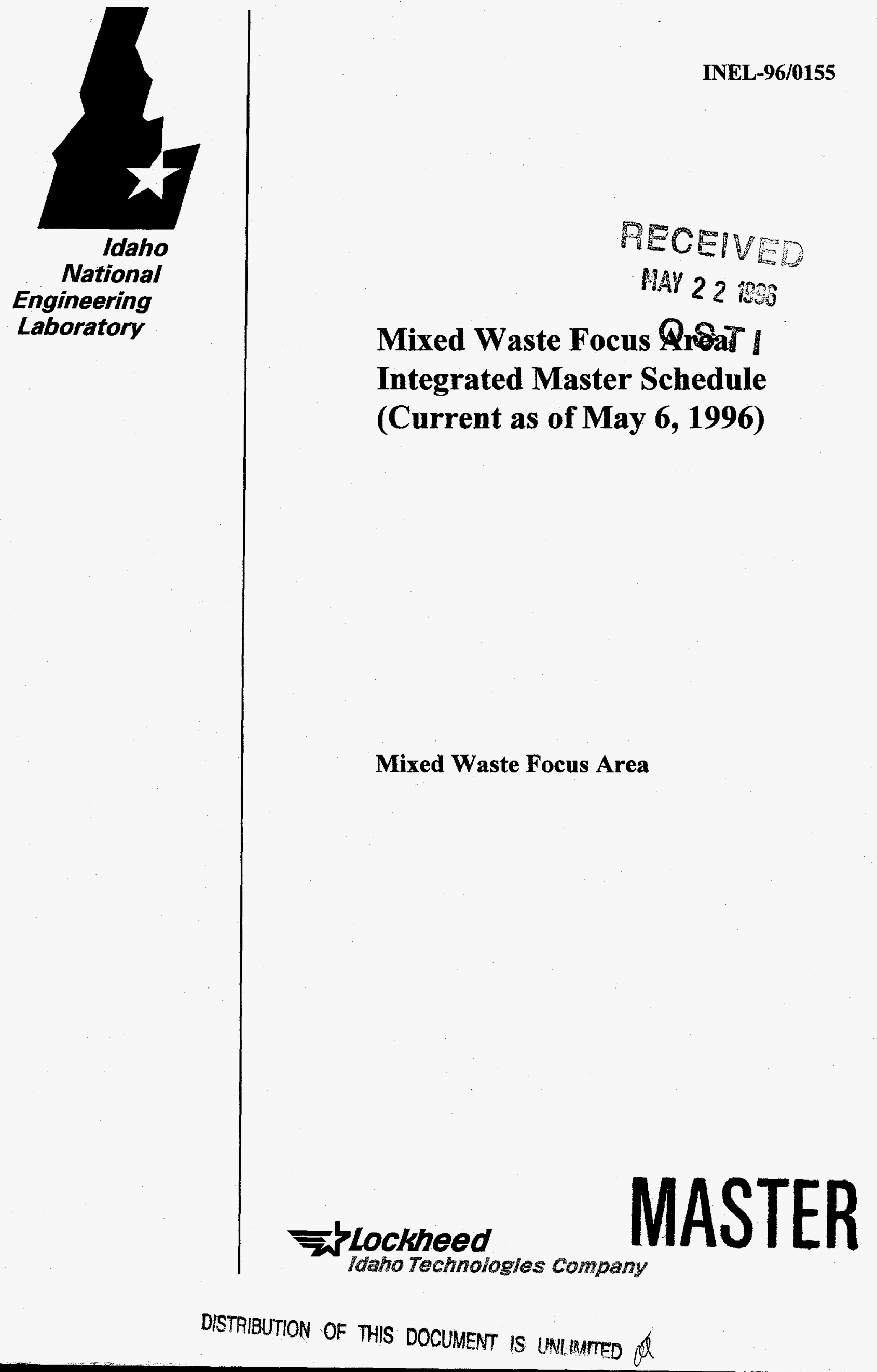


INEL- $-96 / 0155$

INEL-96/0155

\section{Mixed Waste Focus Area Integrated Master Schedule (Current as of May 6, 1996)}

\section{DISCLAIMER}

This report was prepared as an account of work sponsored by an agency of the United States Government. Neither the United States Government nor any agency thereof, nor any of their employees, makes any warranty, express or implied, or assumes any legal liability or responsibility for the accuracy, completeness, or usefulness of any information, apparatus, product, or process disclosed, or represents that its use would not infringe privately owned rights. Reference herein to any specific commercial product, process, or service by trade name, trademark, manufacturer, or otherwise does not necessarily constitute or imply its endorsement, recommendation, or favoring by the United States Government or any agency thereof. The views and opinions of authors expressed herein do not necessarily state or reflect those of the United States Government or any agency thereof.

Published May 1996

Idaho National Engineering LaLoratory Lockheed Idaho Technologies Company

Idaho Falls, Idaho 83415

Prepared for the U.S. Department of Energy 


\begin{abstract}
The mission of the Mixed Waste Characterization, Treatment, and Disposal Focus Area (MWFA) is to provide acceptable treatment systems, developed in partnership with users and with the participation of stakeholders, tribal governments, and regulators, that are capable of treating the Department of Energy's (DOE's) mixed wastes. The MWFA is targeting funding toward technology development projects that address the current list of deficiencies. A clear connection between the technology development projects and the EM-30 and EM-40 treatment systems that they support is essential for optimizing the MWFA efforts. The purpose of the Integrated Master Schedule (IMS) is to establish and document these connections and to ensure that all technology development activities performed by the MWFA are developed for timely use in those treatment systems.
\end{abstract}




\section{DISCLAMMER}

Portions of this document may be illegible in electronic image products. Images are produced from the best available original document. 
ABSTRACT $\ldots \ldots \ldots \ldots \ldots \ldots \ldots \ldots \ldots \ldots \ldots \ldots \ldots \ldots \ldots \ldots \ldots \ldots \ldots \ldots \ldots$

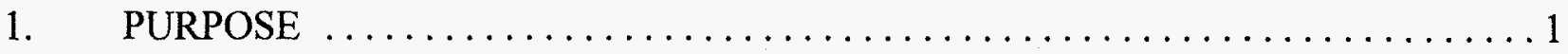

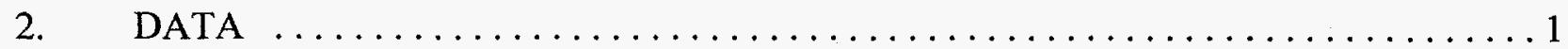

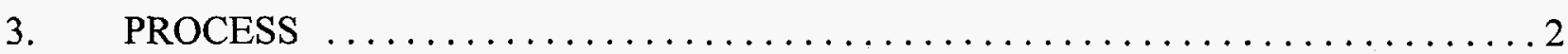

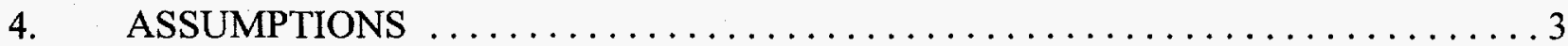

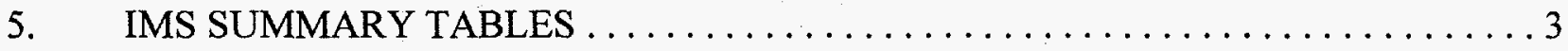

TABLE 1.

TABLE 2.

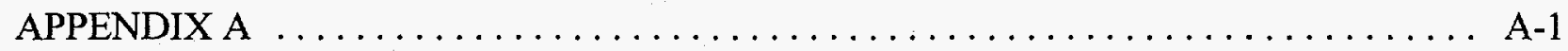

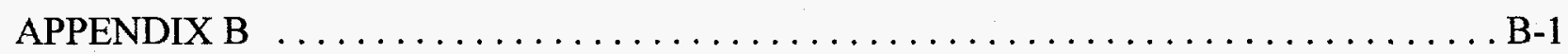

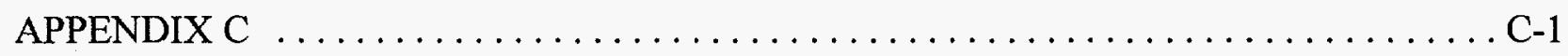

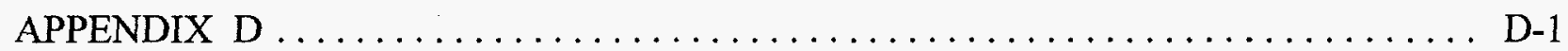




\section{MIXED WASTE FOCUS AREA \\ INTEGRATED MASTER SCHEDULE \\ (Current as of May 6, 1996)}

\section{PURPOSE}

The mission of the Mixed Waste Characterization, Treatment, and Disposal Focus Area (MWFA) is to provide acceptable treatment systems, developed in partnership with users and with the participation of stakeholders, tribal governments, and regulators, that are capable of treating the Department of Energy's (DOE's) mixed wastes. In support of this mission, the MWFA produced the Mixed Waste Focus Area Integrated Technical Baseline Report, Phase 1-Volume 1, January 16, 1996, which identified a prioritized list of 30 national mixed waste technology deficiencies. The MWFA is targeting funding toward technology development projects that address the current list of deficiencies. A clear connection between the technology development projects and the EM-30 and EM-40 treatment systems that they support is essential for optimizing the MWFA efforts. The purpose of the Integrated Master Schedule (IMS) is to establish and document these connections and to ensure that all technology development activities performed by the MWFA are developed for timely use in those treatment systems.

The IMS is a list of treatment systems from the Site Treatment Plans (STPs)/Consent Orders that have been assigned technology development needs with associated time-driven schedules. Technology deficiencies and associated technology development (TD) needs have been identified for each treatment system based on the physical, chemical, and radiological characteristics of the waste targeted for the treatment system. The schedule, the technology development activities, and the treatment system have been verified through the operations contact from the EM-30 organization at the site.

Coordination of TD work and end-user (EM-30/40/60/70) requirements is essential for success of the systematic MWFA multiyear program. The IMS is presently being used to connect the technology development project with end-user planning. Technology Development Requirements Documents are being written and refined based on end-user technical requirements established through this connection.

The IMS is a "living" tool that will be continually updated based on improvements in available data, better understanding of technology development obstacles and solutions, and site needs and input. Updates of the document will be published as deemed useful. For current information contact the MWFA.

\section{DATA}

The facility and waste stream data used for developing the IMS derives from the 1995 Mixed Waste Inventory Report (MWIR) and the 1995 Site Treatment Plan databases. The information in these databases is national-level data provided by all 48 DOE and U.S. Navy sites or shipyards that are storing or planning to treat mixed waste in the future. The STP database identifies the facility, preferred treatment options, and facility compliance dates. The sites develop their national-level waste stream data by combining the best available waste package-level information into general treatability groups and providing an information summary that allows the user to identify treatment technology needs for the 
waste. Since a large quantity of the waste has been in storage for long periods, the waste stream characterization information may not always provide enough information to identify treatment technology needs. As the sites progress in characterizing waste, the information is updated on regular intervals and on an as-needed basis. The 1995 MWIR data was last updated in September 1995. The STP database was last updated in December 1995. Site specific changes have been provided to the MWFA during the spring of 1996 as the sites have completed additional characterization efforts.

\section{PROCESS}

The primary purpose of the IMS is to match the 30 technology development efforts to the needs of the end-users. The first step in developing the IMS was identifying potential TD needs for each of the treatment systems listed in the STP. Each of the 173 treatment systems were reviewed to identify potential TD needs. In total, 173 treatment systems and 2,158 mixed low-level waste (MLLW) and mixed transuranic waste (MTRU) streams, with a combined inventory of approximately $167,000 \mathrm{~m}^{3}$ of waste, were evaluated.

The next step was identifying the date that each end-user needed the technology development completed. Estimated hot operation dates were assigned to each system based on the compliance dates identified in the STP. When dates were not listed in the STP, an estimate was obtained from a knowledgeable site contact. To assist the MWFA in determining the schedule associated with each TD, the amount of time required to develop a solution TD was estimated by the MWFA Technical Resource Team. In the IMS, these estimates are referred to as development time. The development time was reviewed and further refined during the Request for Information (RFI) review process. It was assumed some amount of time would be required to integrate the technology into the individual sites' treatment plan, and include it in permit or regulatory documents. This time period was defined as implementation time and was established by regulatory and other specialists in the MWFA. The technology delivery date was calculated by subtracting the implementation time from the estimated hot operations date. The date the TD activity should start to provide the technology on time was calculated by subtracting the development time from the technology delivery date.

The next step was reviewing the remaining treatment systems and eliminating systems that the MWFA could not support. Reasons for exclusion were that the system was currently operational and had no TD needs, it planned to become operational before the MWFA could provide timely support (i.e., the MWFA could not meet technical development, "window of opportunity") or required simple or existing technology, it was a generator treatment plan, or it operated on a very small scale.

In early April, the draft IMS was distributed to Site representatives for review and comment. Site representatives were asked to verify that the list of site facilities was valid, to update the estimated hot operations start dates, to review and modify the assignment of TD, and to comment on the estimated development and implementation time. These changes were entered into the IMS.

Throughout the development of the IMS, internal reviews were conducted by the Waste Type Teams and Site contacts. A number of systems were reintroduced after management review and site comments. The most striking result of the review process was the drop in the number of treatment systems that the MWFA proposed to support from 173 to 57 , with the associated volume of targeted waste dropping from approximately $167,000 \mathrm{~m}^{3}$ to $123,000 \mathrm{~m}^{3}$. A complete list of treatment systems is included as Appendix A. 


\section{ASSUMPTIONS}

Building the IMS required accepting a number of assumptions. Because the MWIR contains detailed waste stream data, it was used as the waste stream starting point. Preferred treatment options for each waste stream, treatment system data, and limited waste stream data were contained in the STP. To make use of both data sets it was necessary to link the two. This was easily accomplished because the STP was originally populated with MWIR data. More than $95 \%$ of the waste streams reported in the MWIR were reported in the STP. During the time period between populating the MWIR and the STP, a few sites redefined some waste streams. A few waste streams that were reported in the MWIR were not reported in the STP. In most cases, the waste was reported, but under a different waste stream. When MWIR and STP data conflicted, the STP was used because it was more recent then the MWIR. The STP contained a number of waste streams that are intended to track waste inventories through various steps in a treatment train process. For the IMS, all such treatment train streams were excluded. All other streams reported to the STP were used in the analysis.

Waste stream assignment to the treatment system was taken from the STP. To build the IMS, an assumption was made that the waste inventory for a stream would only be counted as input to one facility. For streams targeted to multiple treatment facilities, the inventory of the waste was associated with the primary treatment facility rather than the pre-treatment system.

\section{IMS SUMMARY TABLES}

Two summary tables generated from the IMS data have been provided for your review. Table 1 is a listing of all treatment systems for which the MWFA is attempting to provide TD solutions. Numerous data points are provided for each treatment system. The information listed below provides a description for each field in Table 1.

TS ID -

Site -

System Name -

Targeted Inv $\left(\mathbf{m}^{3}\right)-$

Contact -

Est. Hot Ops Start Date Mortgaged Activities -
Unique number for each treatment system as identified in the STP database.

Site responsible for treatment systems, typically the site where the system will operate. (Appendix B includes a listing of sites and site codes.)

Name of the treatment system from the STP.

The amount of waste targeted to be treated in the system. The inventory information was extracted from the STP database. Some assumptions were made in assigning the targeted wastes. See Section 4 for further clarification.

Name of person who supplied the original data to the MWIR/STP. If the information has been revised by site review, the contact supplying the updated information is listed.

Date the system is expected to start treating mixed waste.

Activities already being funded by the MWFA that are intended to address identified technologies. (Appendix D includes a list and description of mortgaged activities.) 
Included -

Tech Def\# -

Tech Def Name -

TD Should Start By -

Est Dev Time (mo) -

TD Due Date -

Est TD Impl. (mo) -
Whether the system is being targeted by the targeted MWFA.

Technology deficiency identification number.

Name of technology deficiency. (See Appendix C for descriptions of the deficiency.)

The date the TD effort should start was calculated based on subtracting the estimated development and implementation time from the hot ops start date. In some cases, the site provided a required start date. Site-supplied dates are followed by an asterisk $\left({ }^{*}\right)$.

The number of months estimated for developing a solution for the technology deficiency.

The date the TD solution transfers to the treatment system for implementation. For most deficiencies, this date was calculated by subtracting the estimated TD implementation time from the hot ops start date. In some cases, the site provided a required TD due date. Site-supplied dates are followed by an asterisk $(*)$.

Minimum time required by the site to implement the TD solution into the proposed treatment process.

Table 2 provides the list of treatment systems associated with each TD. The information listed below provides a description for each field in Table 2 .

Tech Def \# -

Tech Def Name -

TD Dev Time (mo) -

TD Imp Time (mo) -

TS ID -

System Name -

Site -

Targeted Inv $\left(\mathrm{m}^{3}\right)-$
Technology deficiency identification number.

Name of technology deficiency. (See Appendix C for descriptions of the deficiency.)

The length of time estimated by the Technical Resource Team to develop a solution for the deficiency.

The estimated length of time required to implement the technology into the Site Treatment Plan after completion of technology development.

Unique number for each treatment system as identified in the STP database.

Treatment system name from the STP.

Site responsible for treatment systems, typically the site where the system will operate.

The amount of waste targeted to be treated in the system. The inventory information was extracted from the STP database. Some assumptions were 
made in assigning the targeted wastes. See Section 4 for further clarification.

TD Should Start By - The date the TD effort should start was calculated based on subtracting the estimated development and implementation time from the hot ops start date. In some cases, the site provided a required start date. Site-supplied dates are followed by an asterisk $(*)$.

Est. Hot Ops Start Date - Date the system is expected to start treating mixed waste. 
TABLE 1.

\section{IMS - Technology Deficiencies Assigned to Treatment Systems}

\begin{tabular}{|c|c|c|c|c|c|c|c|}
\hline \multirow{2}{*}{$\begin{array}{l}\text { TS ID } \\
\text { AW-S007 }\end{array}$} & \multirow{3}{*}{ Site } & System Name & \multicolumn{2}{|c|}{ Targeted Inv $\left(\mathrm{m}^{3}\right)$} & Contact & \multicolumn{2}{|c|}{ Est. Hot Ops Start Date } \\
\hline & & Remote Treatment Facility (RTF) & MLLW: & 44.45 & irt, Nancy & & $9 / 30 / 2007$ \\
\hline & & & MTRU: & 13.88 & & & \\
\hline \multicolumn{3}{|c|}{ Mortgaged Activities: } & Included : & Yes & & & \\
\hline \multicolumn{2}{|c|}{ Tech Def \# } & Tech Def Name & TD Should Start By & $\begin{array}{c}\text { Est. } \\
\text { Dev Time (mo) }\end{array}$ & TD Due Date & $\begin{array}{l}\text { Est. } \\
\text { TD Impl. (mo) }\end{array}$ & \\
\hline \multirow[t]{2}{*}{ BN-S701 } & $\mathrm{BN}$ & Characterize to Determine Hazardous Constituent & MLLW: & 16.4 & a, Glen & & \\
\hline & & & MTRU: & 0 & & & \\
\hline \multicolumn{3}{|c|}{ Mortgaged Activities: } & Included : & Yes & & & \\
\hline \multicolumn{2}{|c|}{ Tech Def \# } & Tech Def Name & TD Should Start By & $\begin{array}{c}\text { Est. } \\
\text { Dev Time (mo) }\end{array}$ & TD Due Date & $\begin{array}{l}\text { Est. } \\
\text { TD Impl. (mo) }\end{array}$ & \\
\hline \multirow[t]{2}{*}{ DP-S001 } & DP & Central Neutralization Facility & MLLW: & Cor & $y$, Tom & & $12 / 1 / 96$ \\
\hline & & & MTRU: & 0 & & & \\
\hline \multicolumn{3}{|c|}{ Mortgaged Activities: } & Included : & Yes & & & \\
\hline Tec & Def \# & Tech Def Name & TD Should Start By & $\begin{array}{c}\text { Est. } \\
\text { Dev Time (mo) }\end{array}$ & TD Due Date & $\begin{array}{l}\text { Est. } \\
\text { TD Impl. (mo) }\end{array}$ & \\
\hline & 99 & TD assignment being assessed & $12 / 1 / 96$ & 0 & $12 / 1 / 96$ & 0 & \\
\hline
\end{tabular}




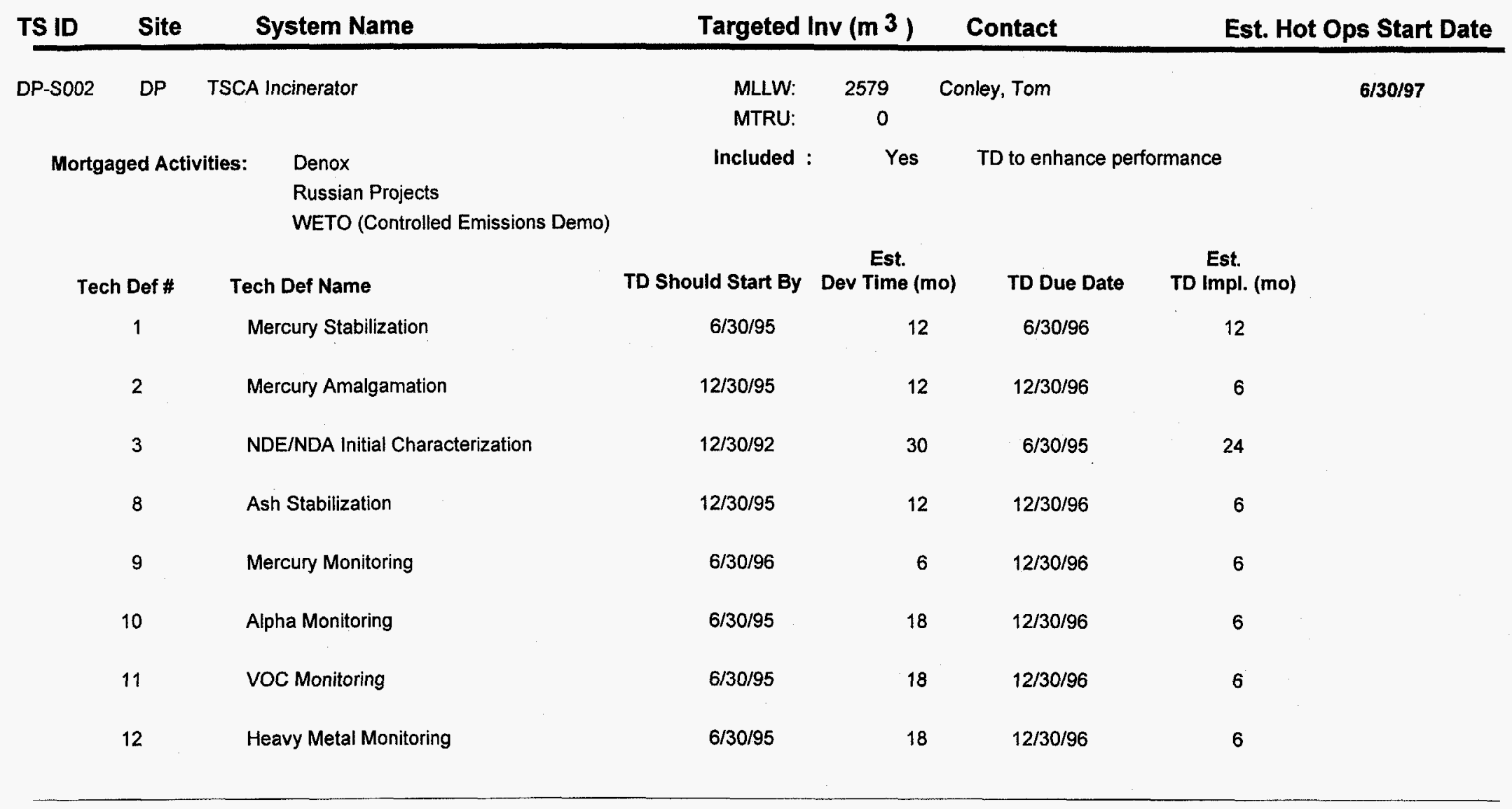




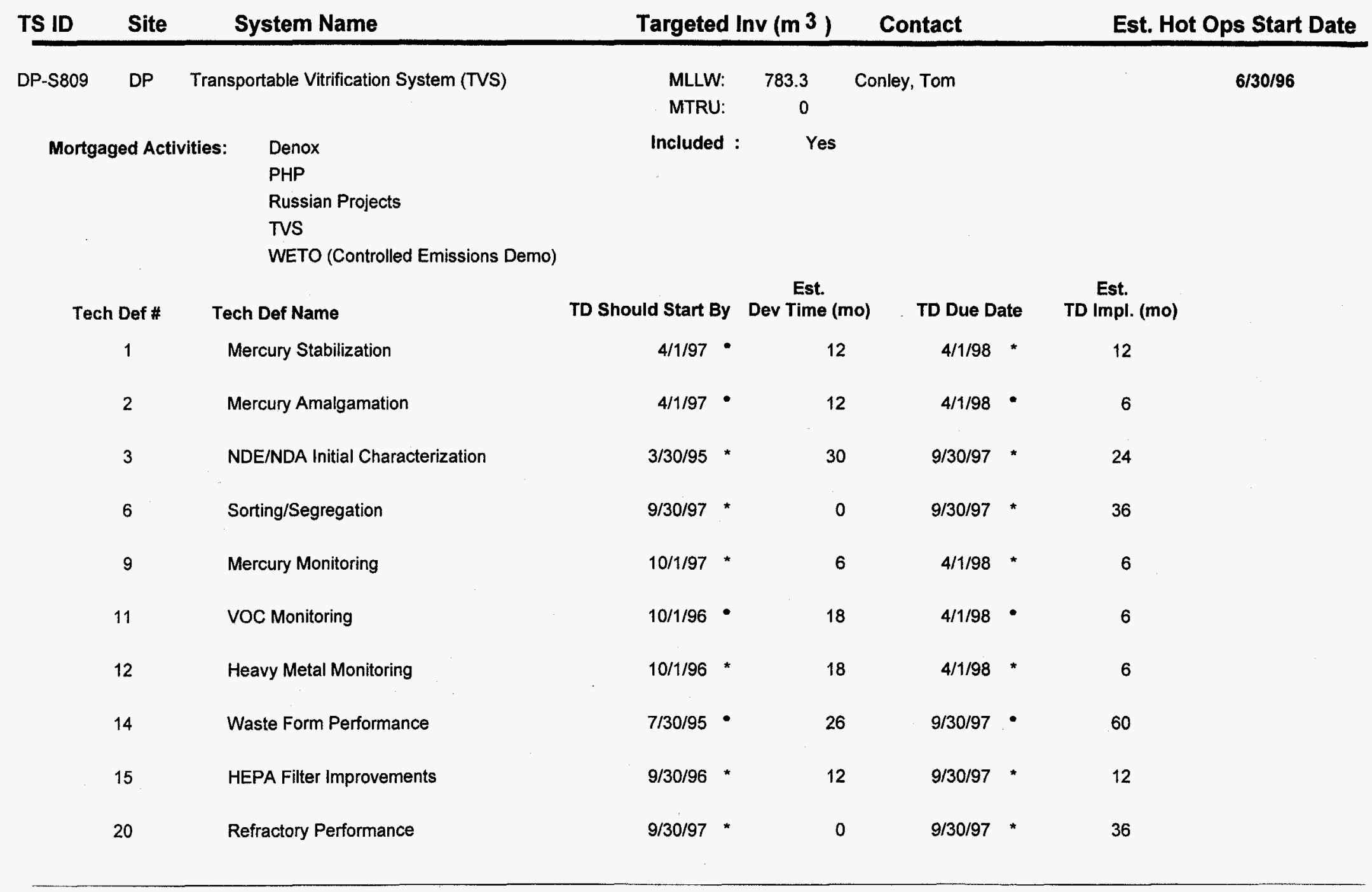




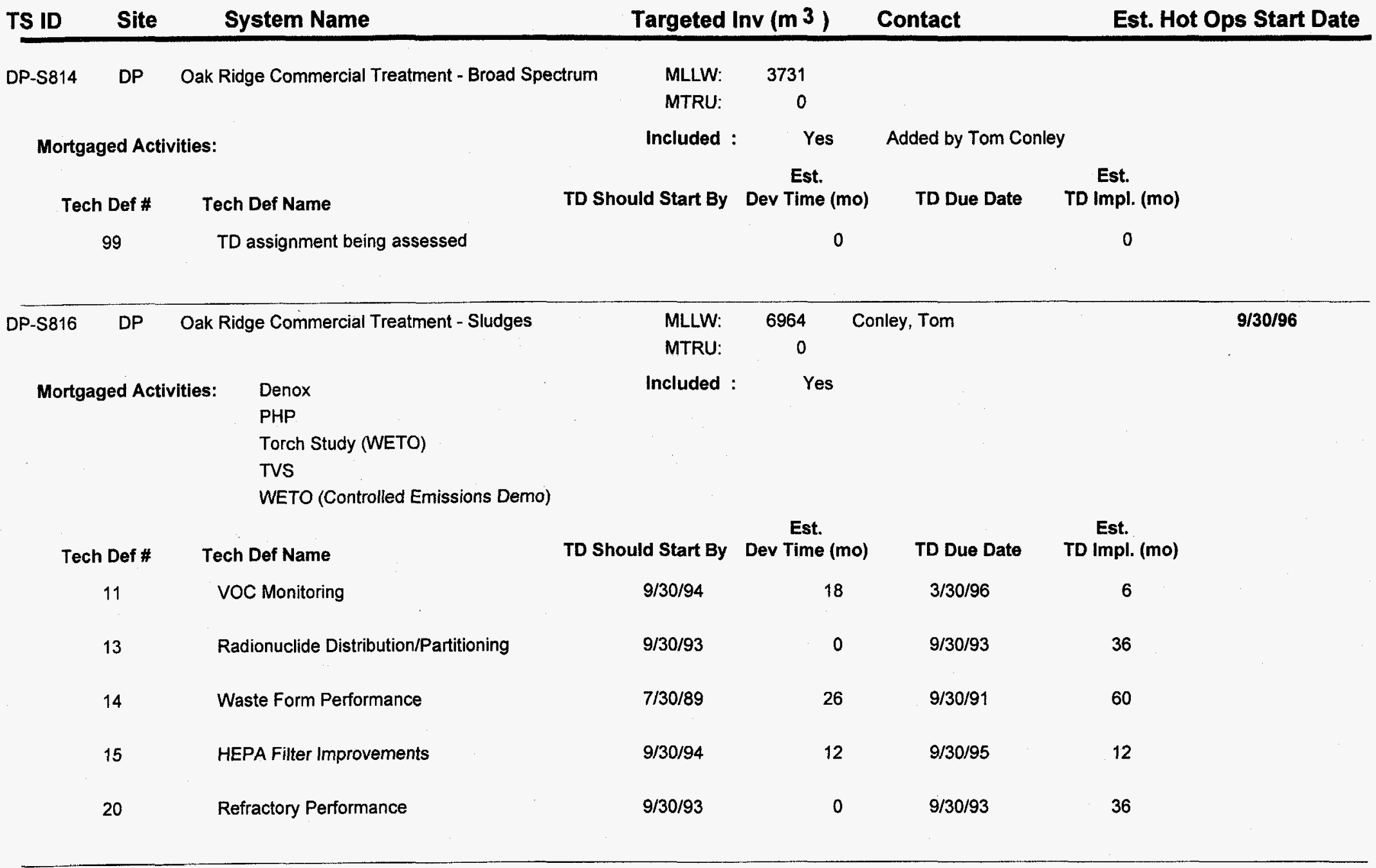




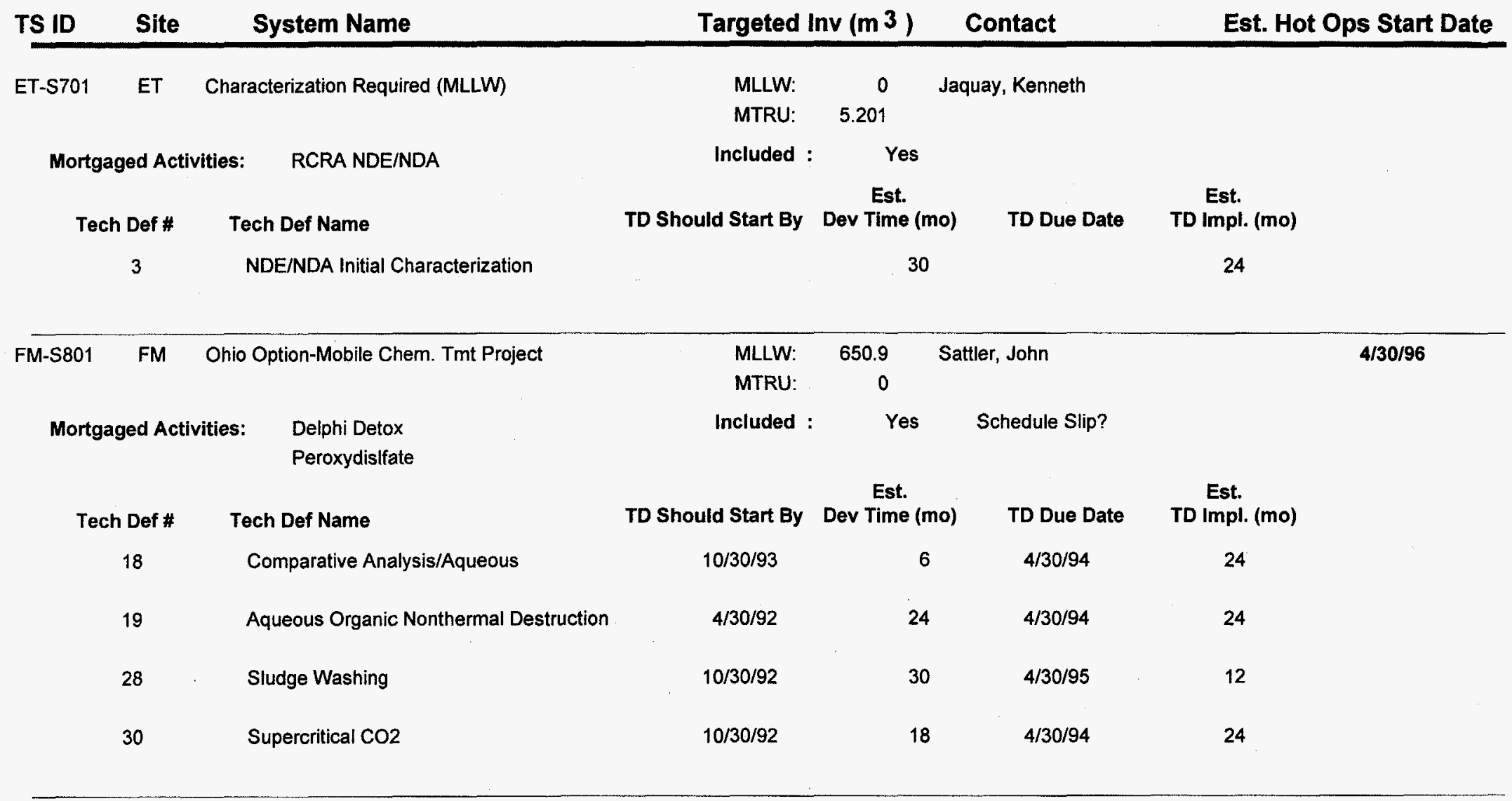




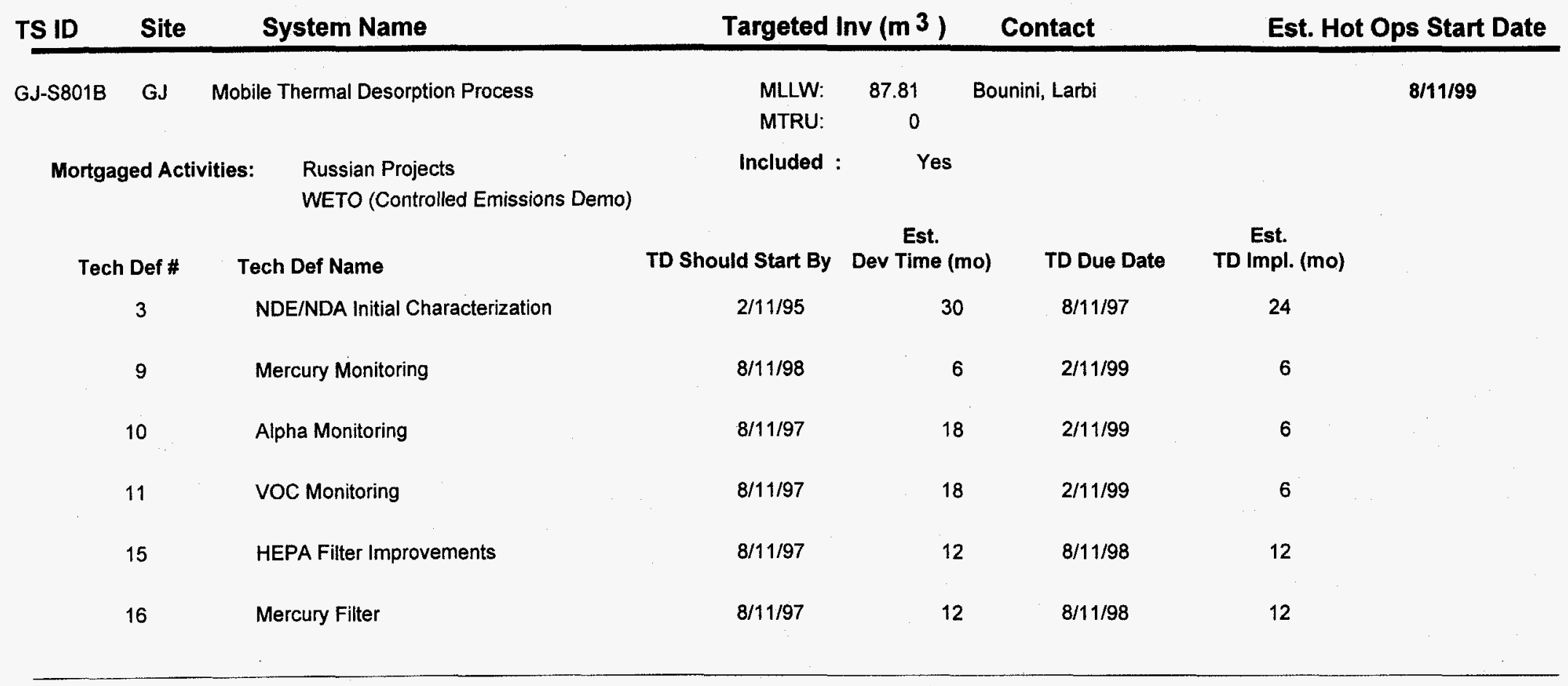


GJ-S801C GJ Mobile Evaporative Oxidation Process

$\begin{array}{ll}\text { Mortgaged Activities: } & \text { Delphi Detox } \\ & \text { Peroxydislfate } \\ & \text { Russian Projects } \\ & \text { WETO (Controlled Emissions Demo) }\end{array}$

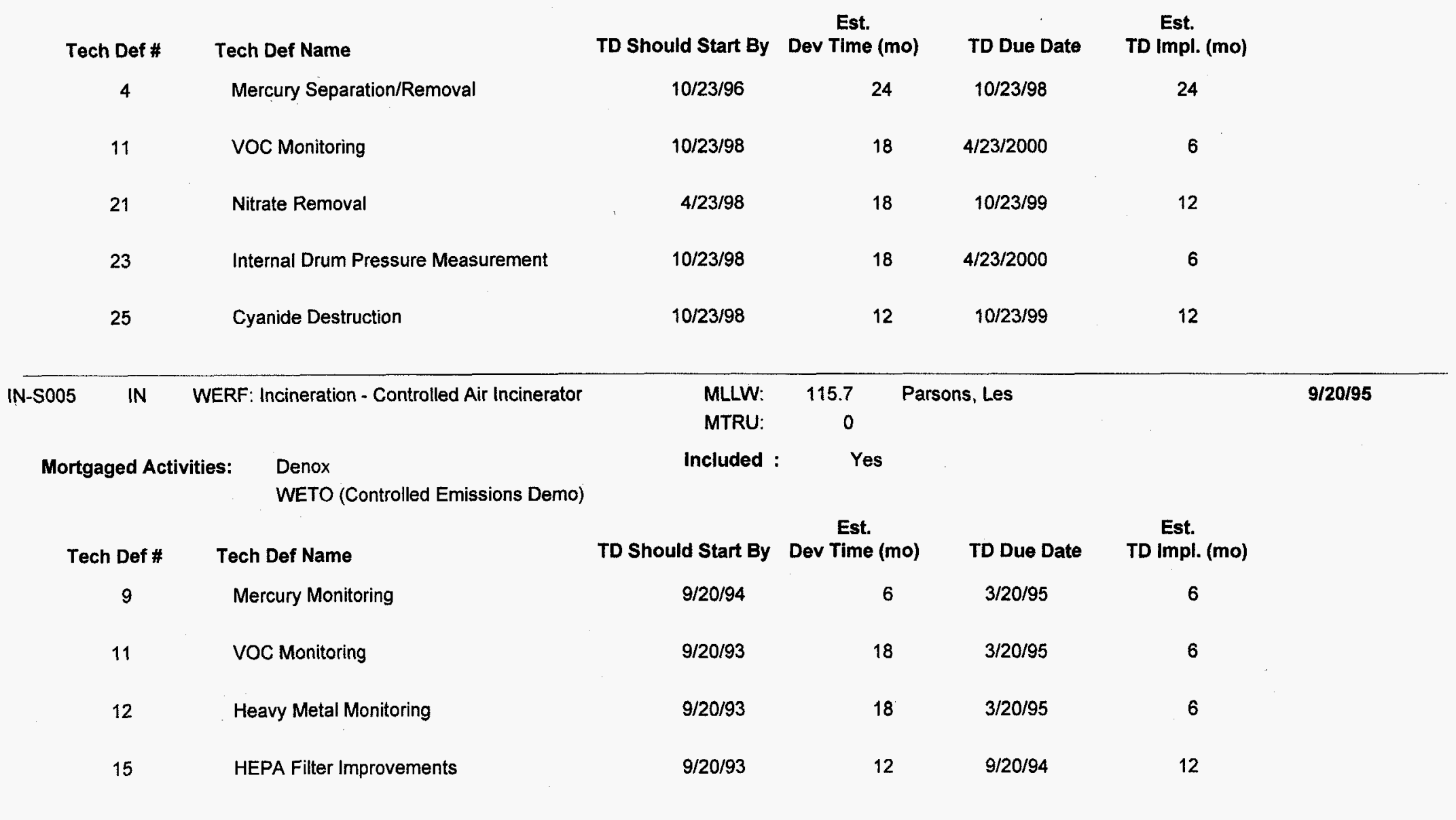

\section{$10 / 23 / 2000$}

Included : Yes 


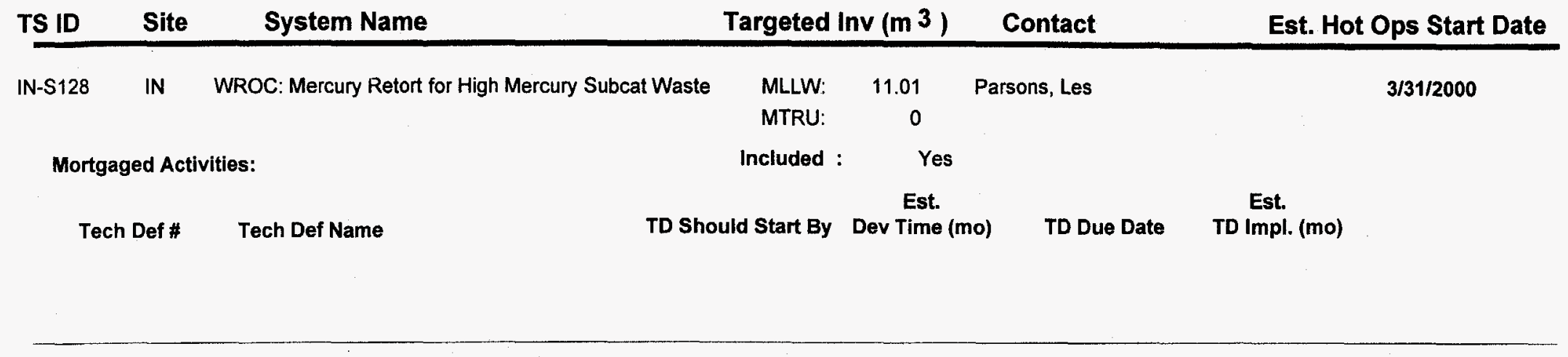




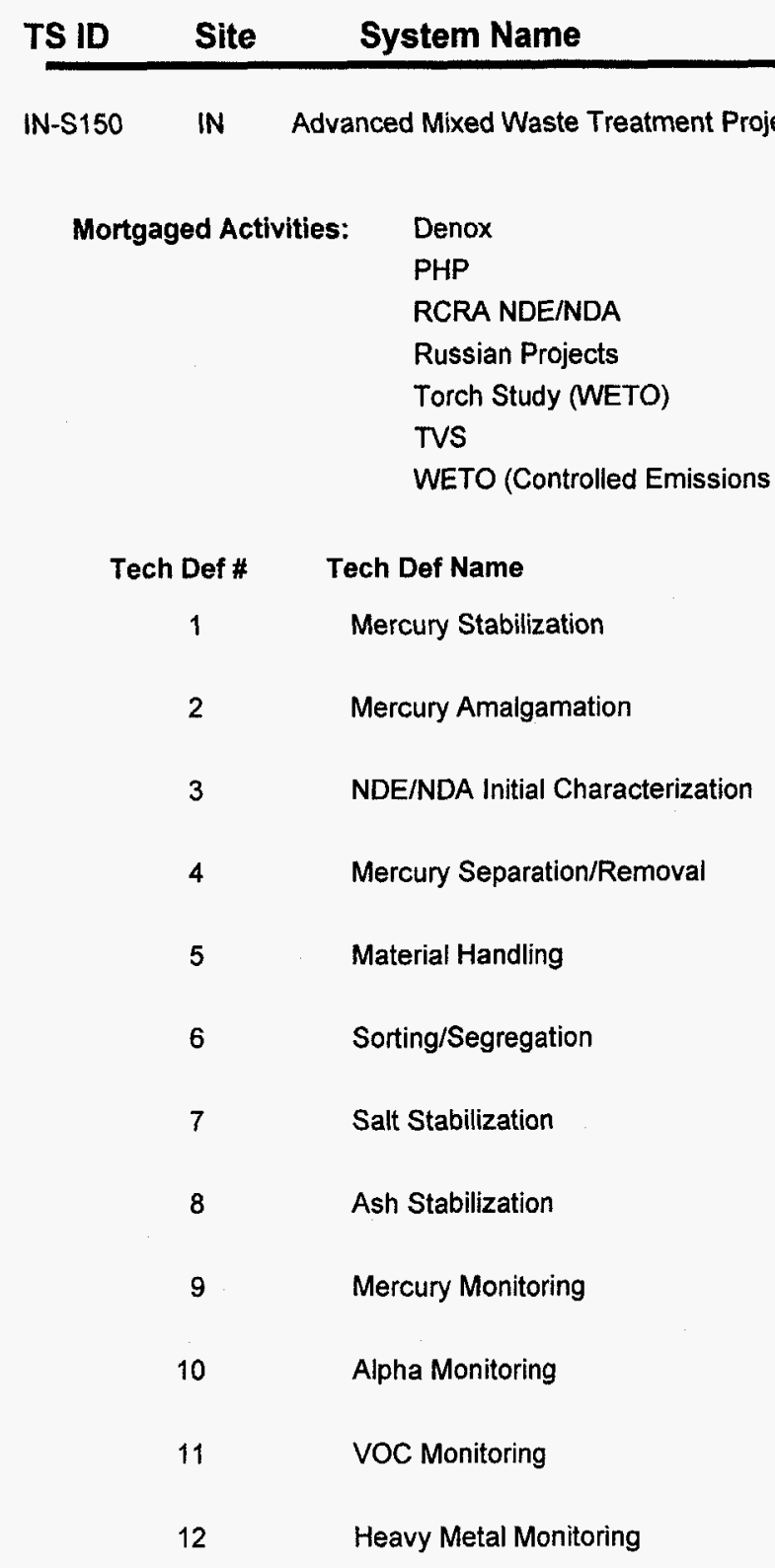

\begin{tabular}{|c|c|c|c|}
\hline MLLW: & 24380 & Anderson, Gary & $6 / 30 / 2002$ \\
\hline MTRU: & 38630 & & \\
\hline
\end{tabular}

Included : $\quad$ Yes Hot Ops Date or Contract Date

Est.

TD Should Start By Dev Time (mo)

$6 / 30 / 2000$
$12 / 30 / 2000$
$12 / 30 / 97$
$6 / 30 / 98$
$6 / 30 / 99$
$6 / 30 / 99$
$12 / 30 / 2000$
$12 / 30 / 2000$
$6 / 30 / 2001$
$6 / 30 / 2000$
$6 / 30 / 2000$
$6 / 30 / 2000$

$6 / 30 / 2000$

$\begin{array}{rr}12 & 6 / 30 / 2001 \\ 12 & 12 / 30 / 2001 \\ 30 & 6 / 30 / 2000 \\ 24 & 6 / 30 / 2000 \\ 0 & 6 / 30 / 99 \\ 0 & 6 / 30 / 99 \\ 12 & 12 / 30 / 2001 \\ 12 & 12 / 30 / 2001 \\ 6 & 12 / 30 / 2001 \\ 18 & 12 / 30 / 2001 \\ 18 & 12 / 30 / 2001 \\ 18 & 12 / 30 / 2001\end{array}$

Est.

TD Impl. (mo)

12

6

24

24

36

36

6

6

6

6

6

6 


\begin{tabular}{|c|c|c|c|c|c|c|}
\hline TS ID & Site & System Name & Targeted 1 & $\ln v\left(\mathrm{~m}^{3}\right)$ & Contact & Est. Hot Ops Start Date \\
\hline & 13 & Radionuclide Distribution/Partitioning & $6 / 30 / 99$ & 0 & $6 / 30 / 99$ & 36 \\
\hline & 14 & Waste Form Performance & $4 / 30 / 95$ & 26 & $6 / 30 / 97$ & 60 \\
\hline & 15 & HEPA Filter Improvements & $6 / 30 / 2000$ & 12 & $6 / 30 / 2001$ & 12 \\
\hline & 16 & Mercury Filter & $6 / 30 / 2000$ & 12 & $6 / 30 / 2001$ & 12 \\
\hline & 17 & Molten Product - Recanting & $6 / 30 / 99$ & 0 & $6 / 30 / 99$ & 36 \\
\hline & 20 & Refractory Performance & $6 / 30 / 99$ & 0 & $6 / 30 / 99$ & 36 \\
\hline IN-S151 & IN & Remote Treatment Facility - RH Preparation Unit & MLLW: & 0 & & $12 / 31 / 2007$ \\
\hline & & & MTRU: & 0 & & \\
\hline Tec & Def \# & Tech Def Name & TD Should Start By & $\begin{array}{c}\text { Est. } \\
\text { Dev Time (mo) }\end{array}$ & TD Due Date & $\begin{array}{l}\text { Est. } \\
\text { TD Impl. (mo) }\end{array}$ \\
\hline & 3 & NDE/NDA Initial Characterization & $6 / 30 / 2003$ & 30 & $12 / 31 / 2005$ & 24 \\
\hline & 5 & Material Handling & $12 / 31 / 2004$ & 0 & $12 / 31 / 2004$ & 36 \\
\hline & 6 & Sorting/Segregation & $12 / 31 / 2004$ & 0 & $12 / 31 / 2004$ & 36 \\
\hline
\end{tabular}




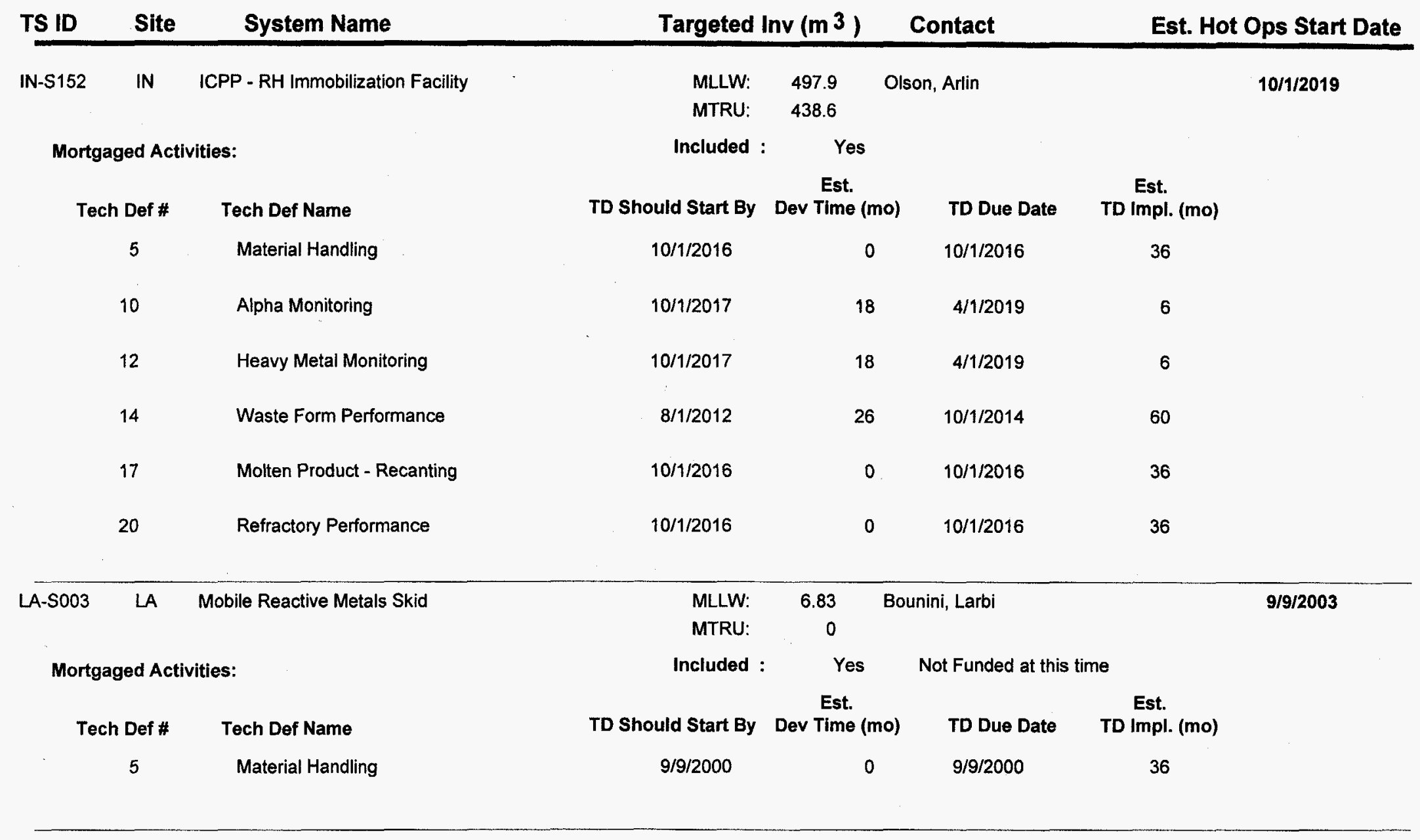




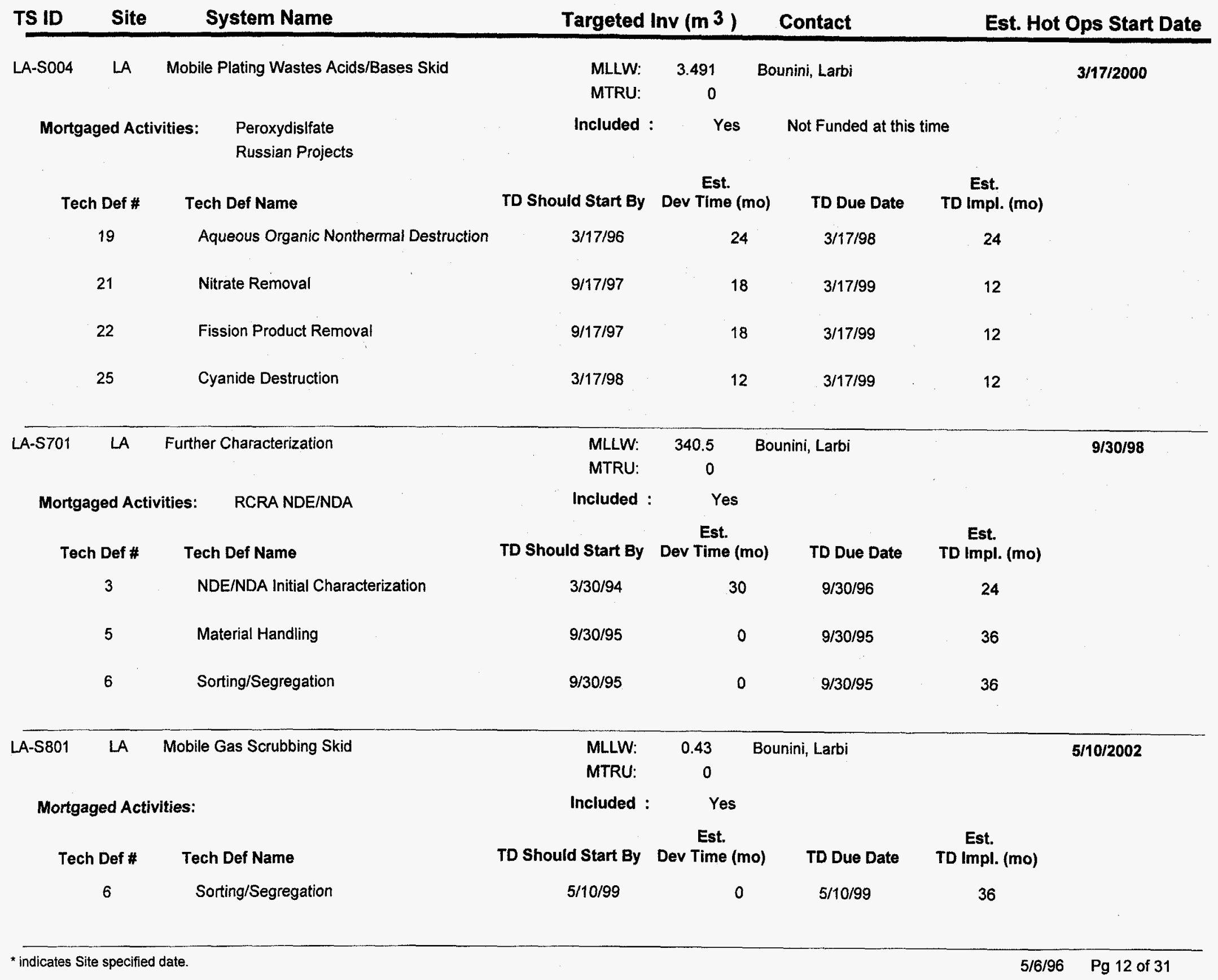




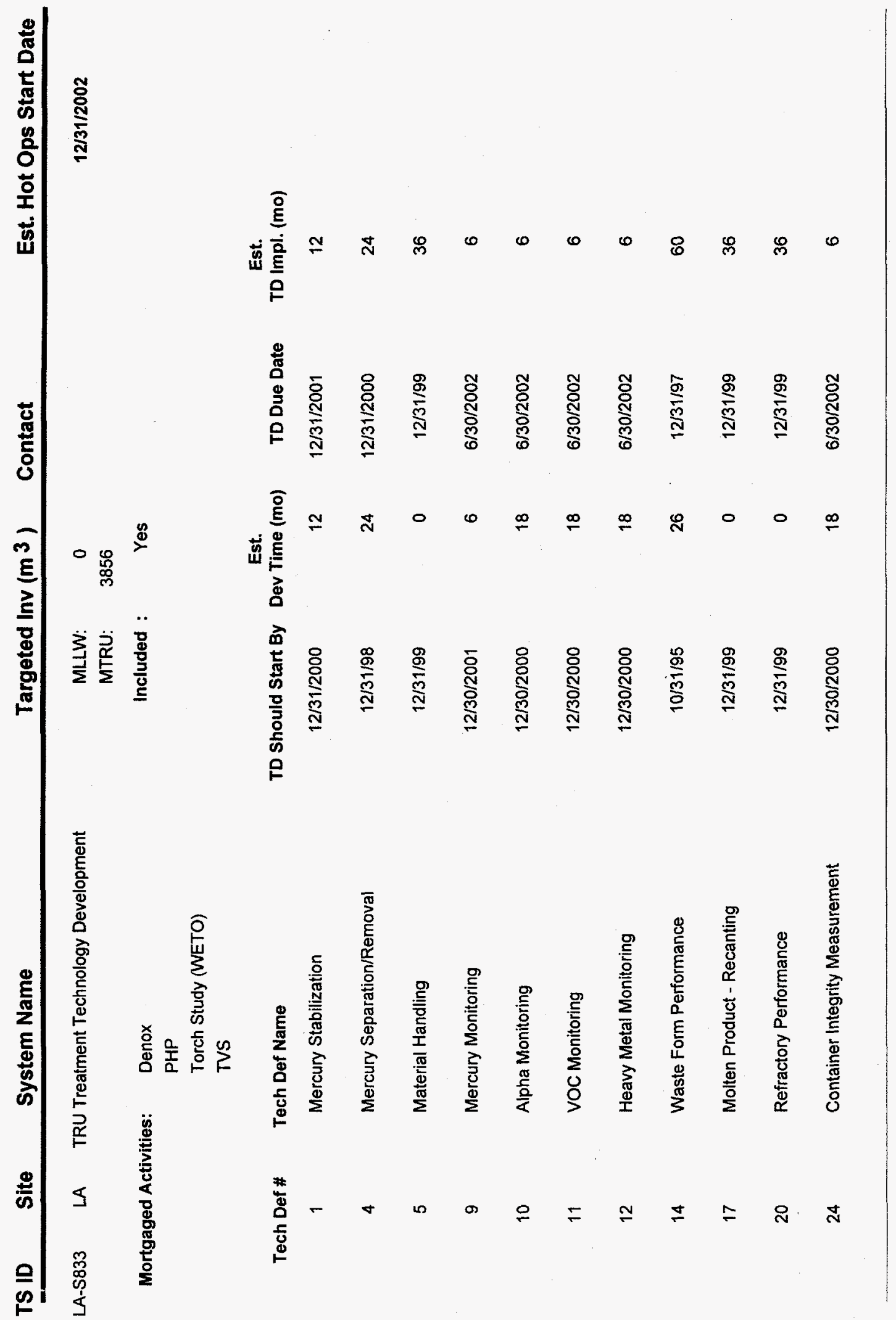




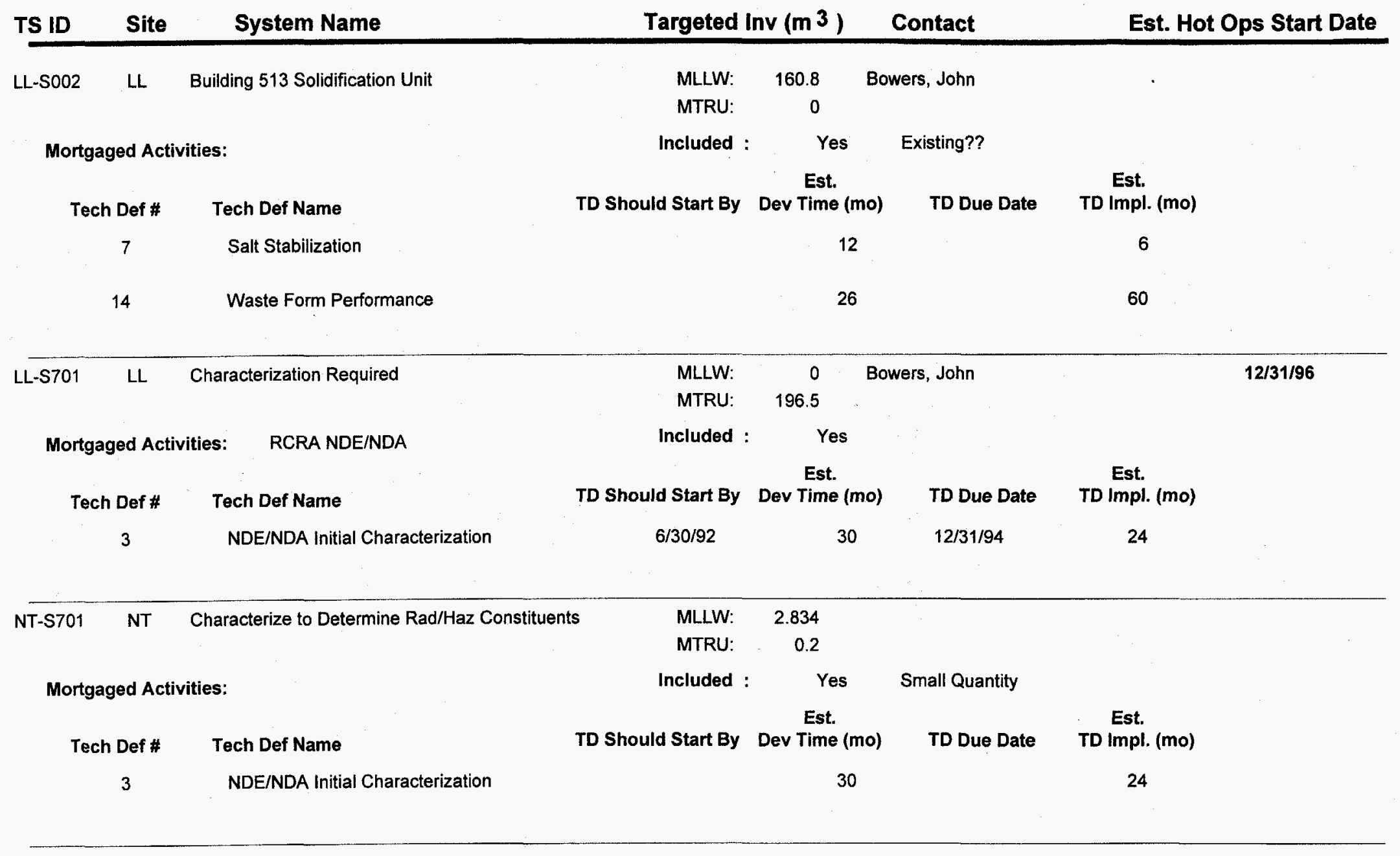




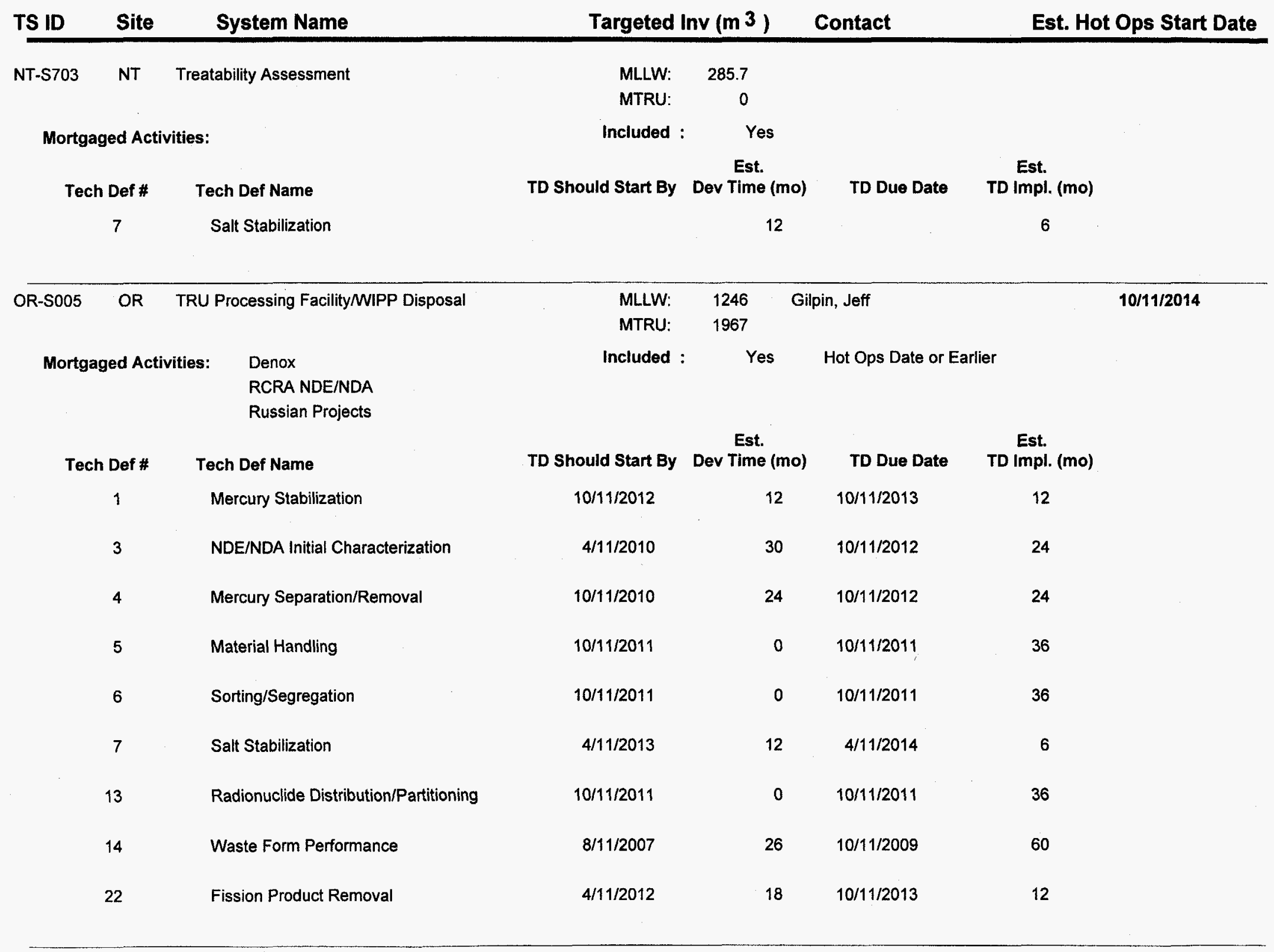




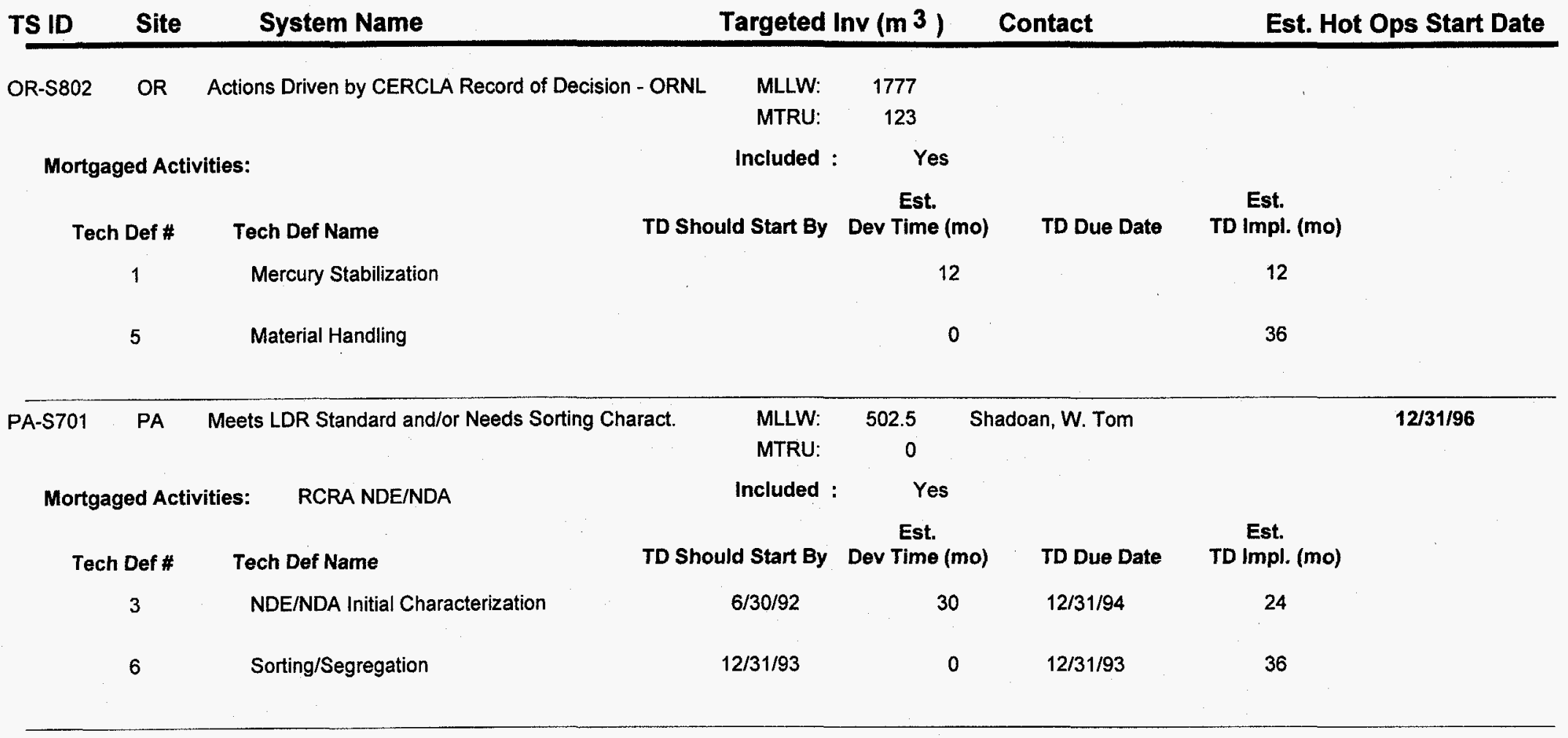




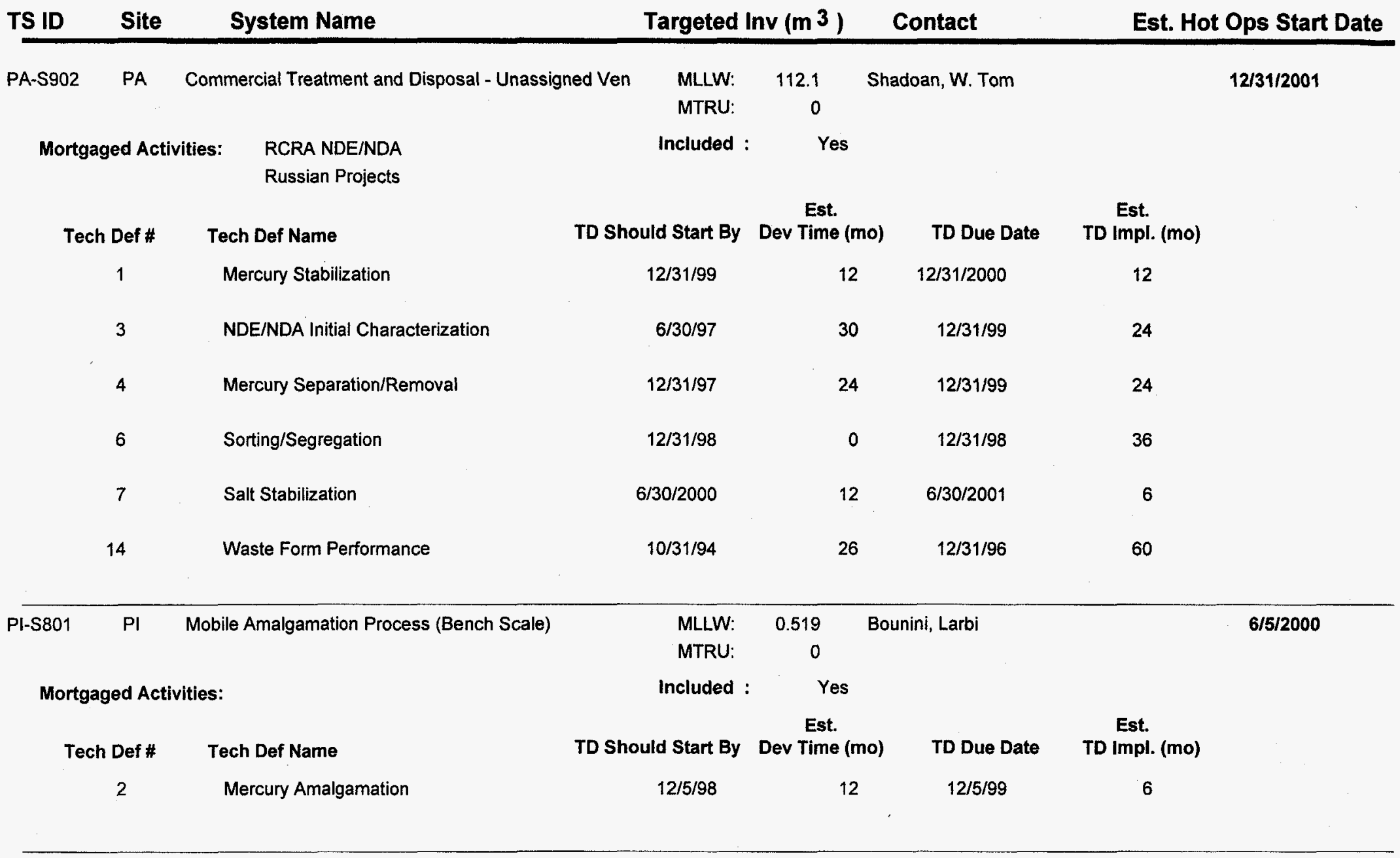




\begin{tabular}{|c|c|c|c|c|c|c|c|}
\hline TS ID & Site & System Name & Targeted I & $\operatorname{lnv}\left(\mathrm{m}^{3}\right)$ & Contact & Est. Ho & Ops Start Date \\
\hline PO-S802 & PO & Pretreatment/Regeneration & MLLW: & 135.5 & Jole, Joanna & & 12/31/2001 \\
\hline & & & MTRU: & 0 & & & \\
\hline Mortga & ged Act & pities: & Included : & Yes & Commercial & & \\
\hline Tec & Def \# & Tech Def Name & TD Should Start By & $\begin{array}{c}\text { Est. } \\
\text { Dev Time (mo) }\end{array}$ & TD Due Date & $\begin{array}{l}\text { Est. } \\
\text { TD Impl. (mo) }\end{array}$ & \\
\hline & 11 & VOC Monitoring & $12 / 30 / 99$ & 18 & $6 / 30 / 2001$ & 6 & \\
\hline & 12 & Heavy Metal Monitoring & $12 / 30 / 99$ & 18 & $6 / 30 / 2001$ & 6 & \\
\hline & 26 & Thermal Desorption & $12 / 31 / 99$ & 0 & $12 / 31 / 99$ & 24 & \\
\hline
\end{tabular}




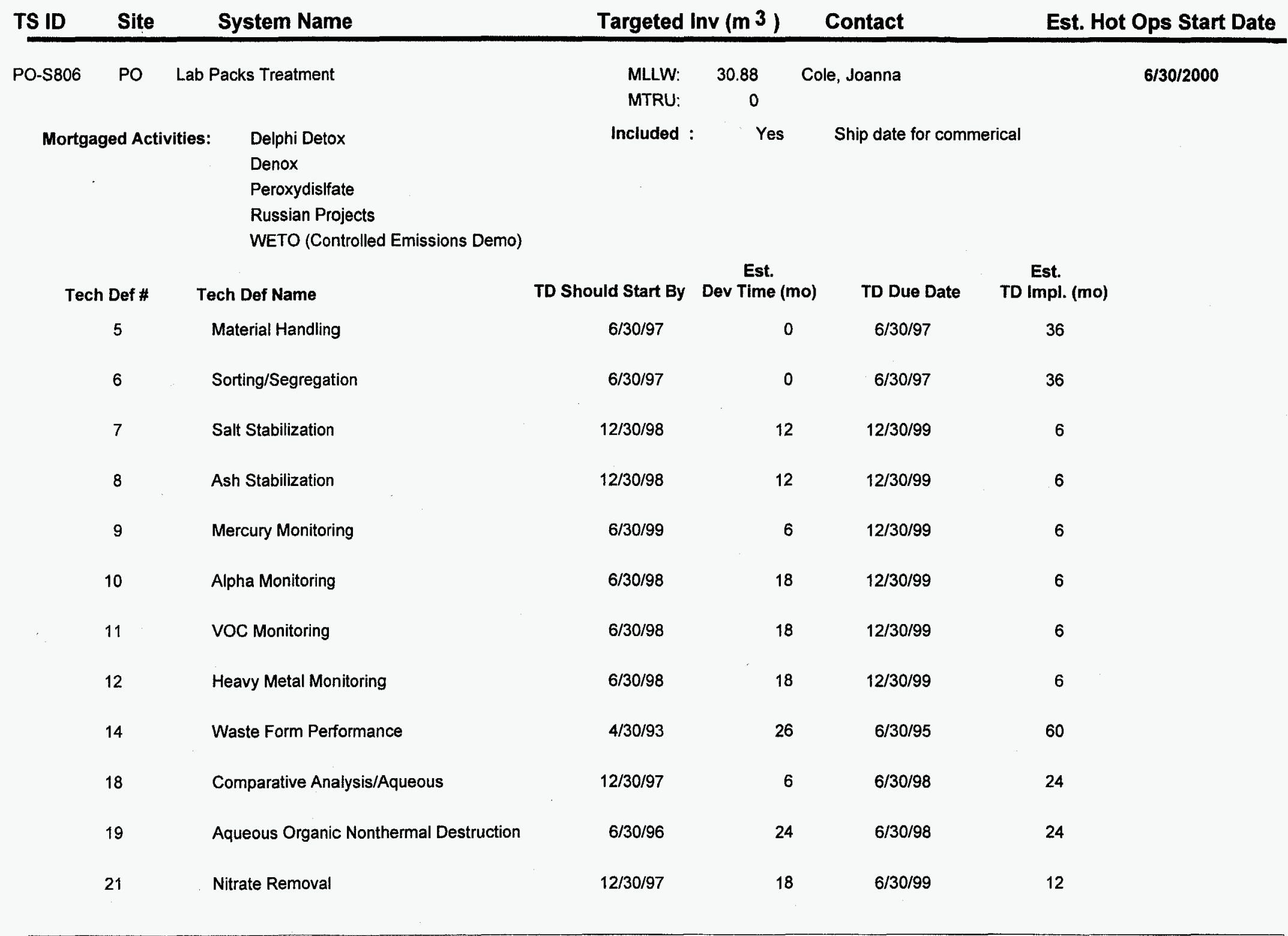




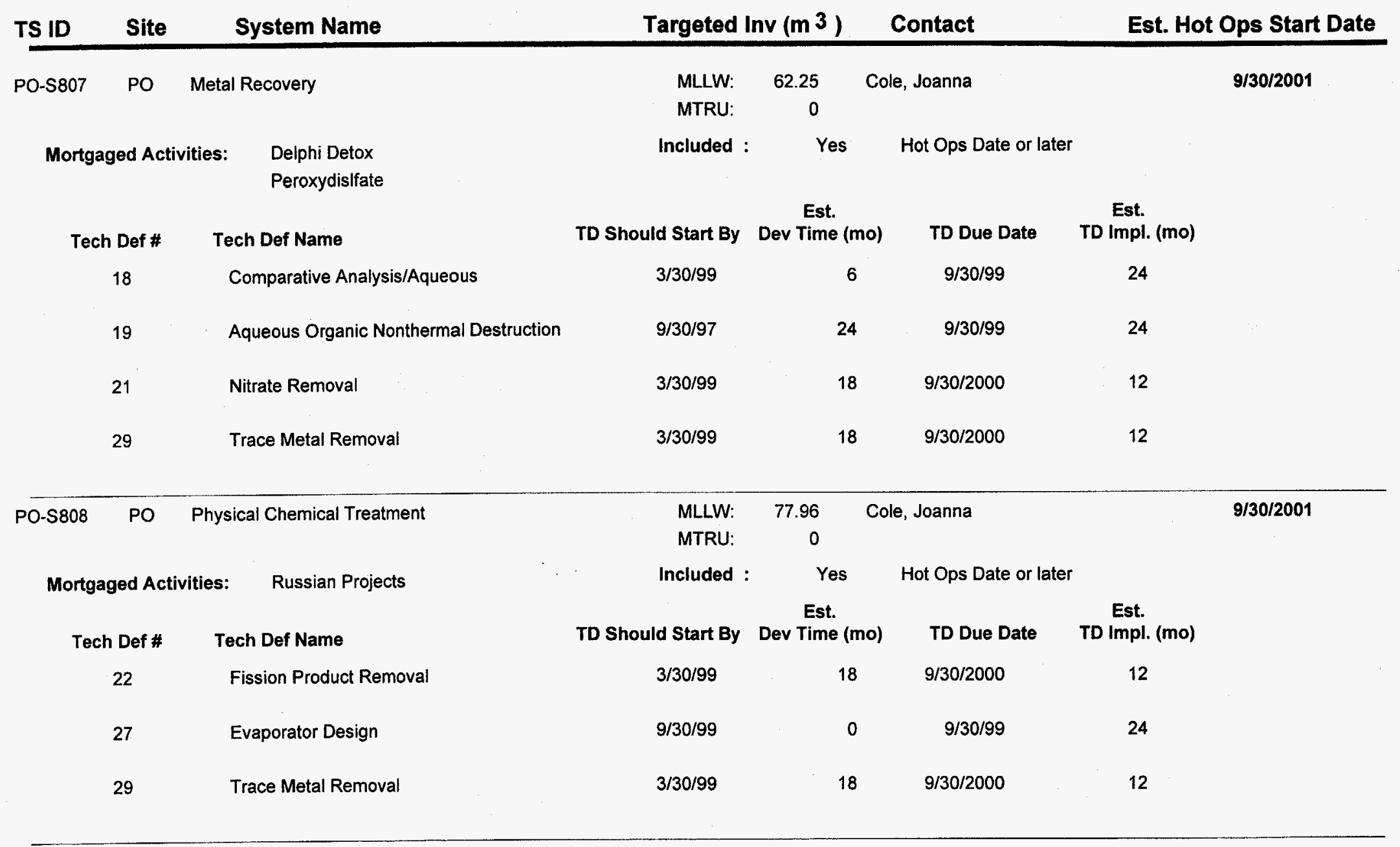




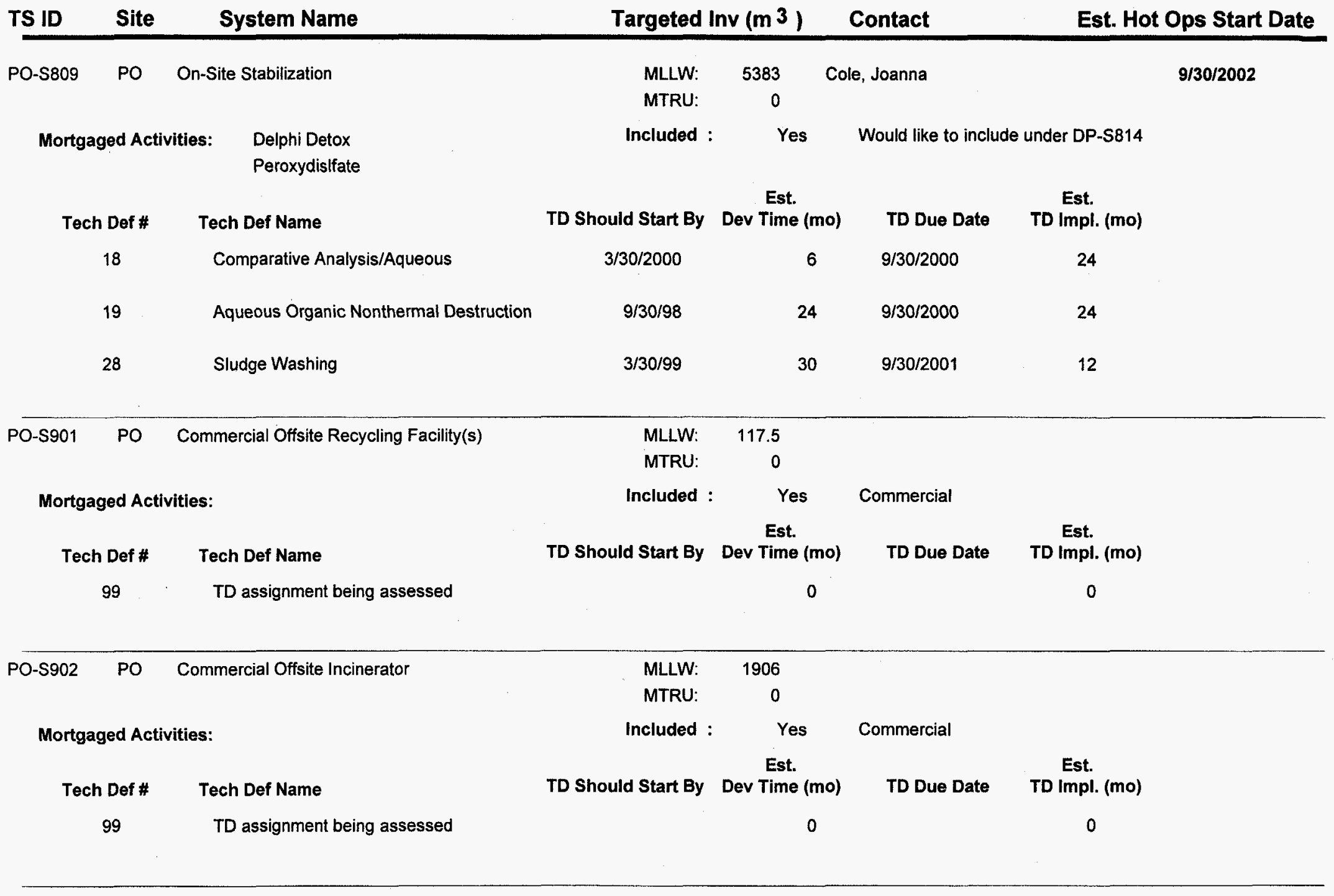




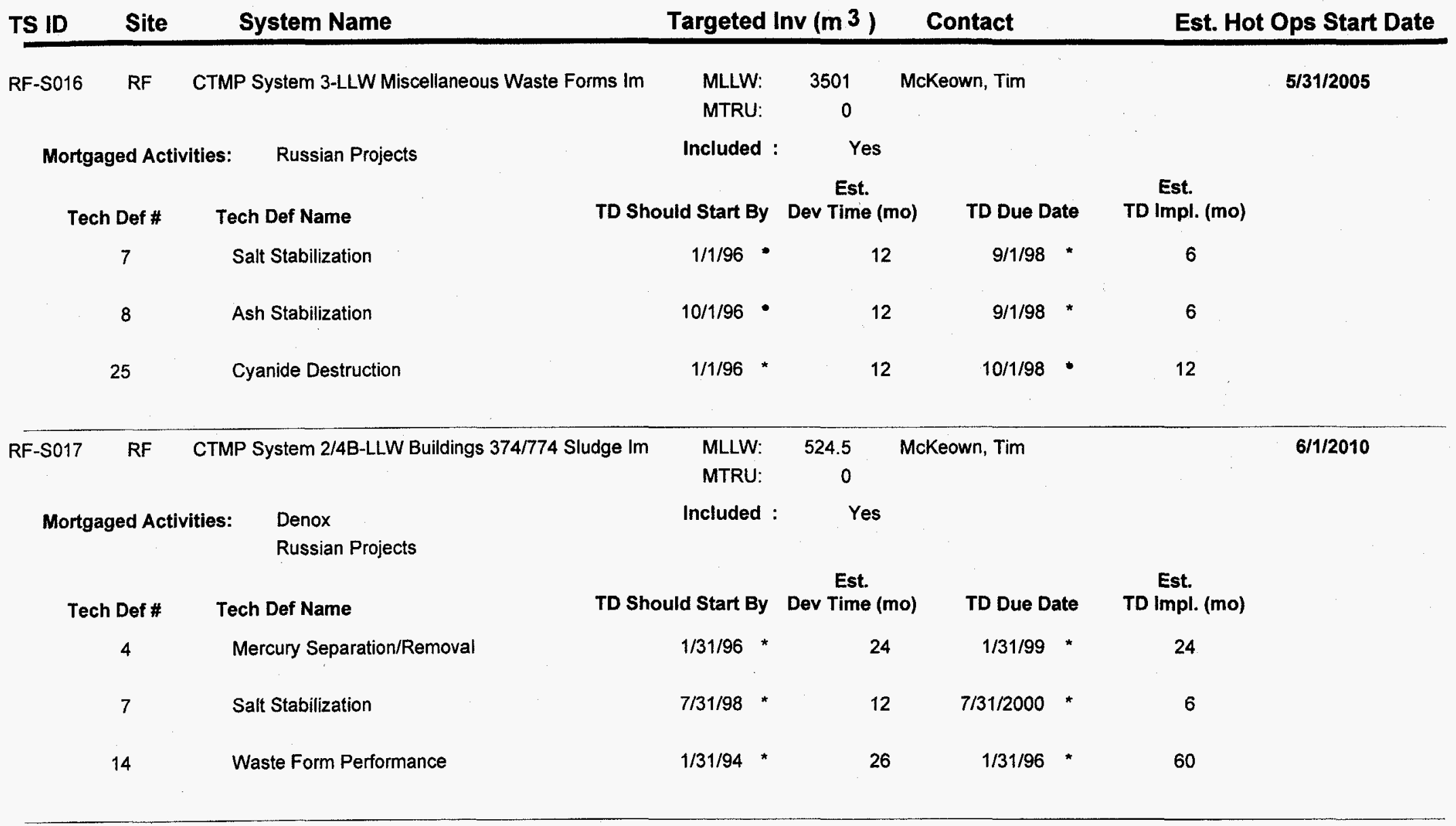




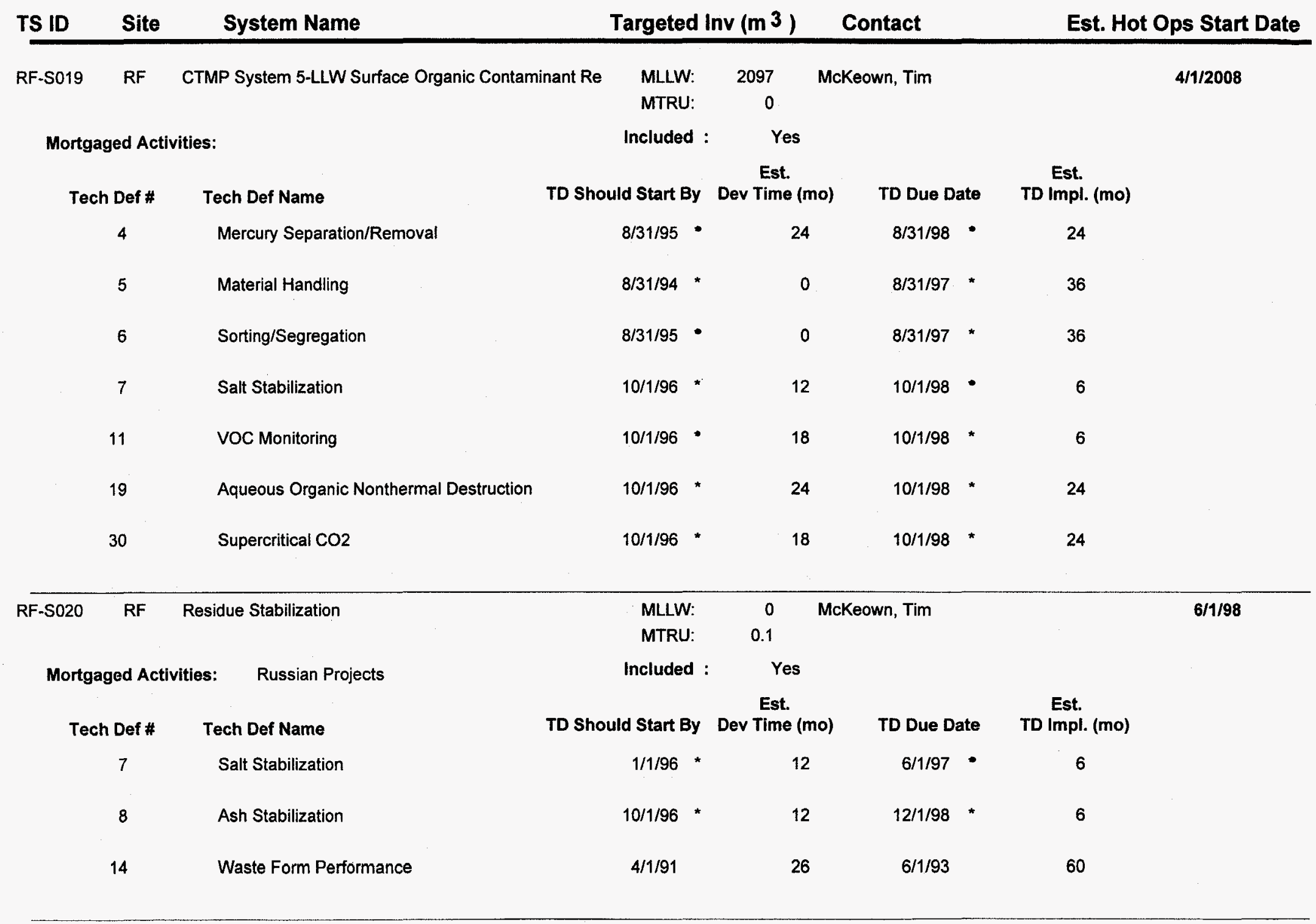




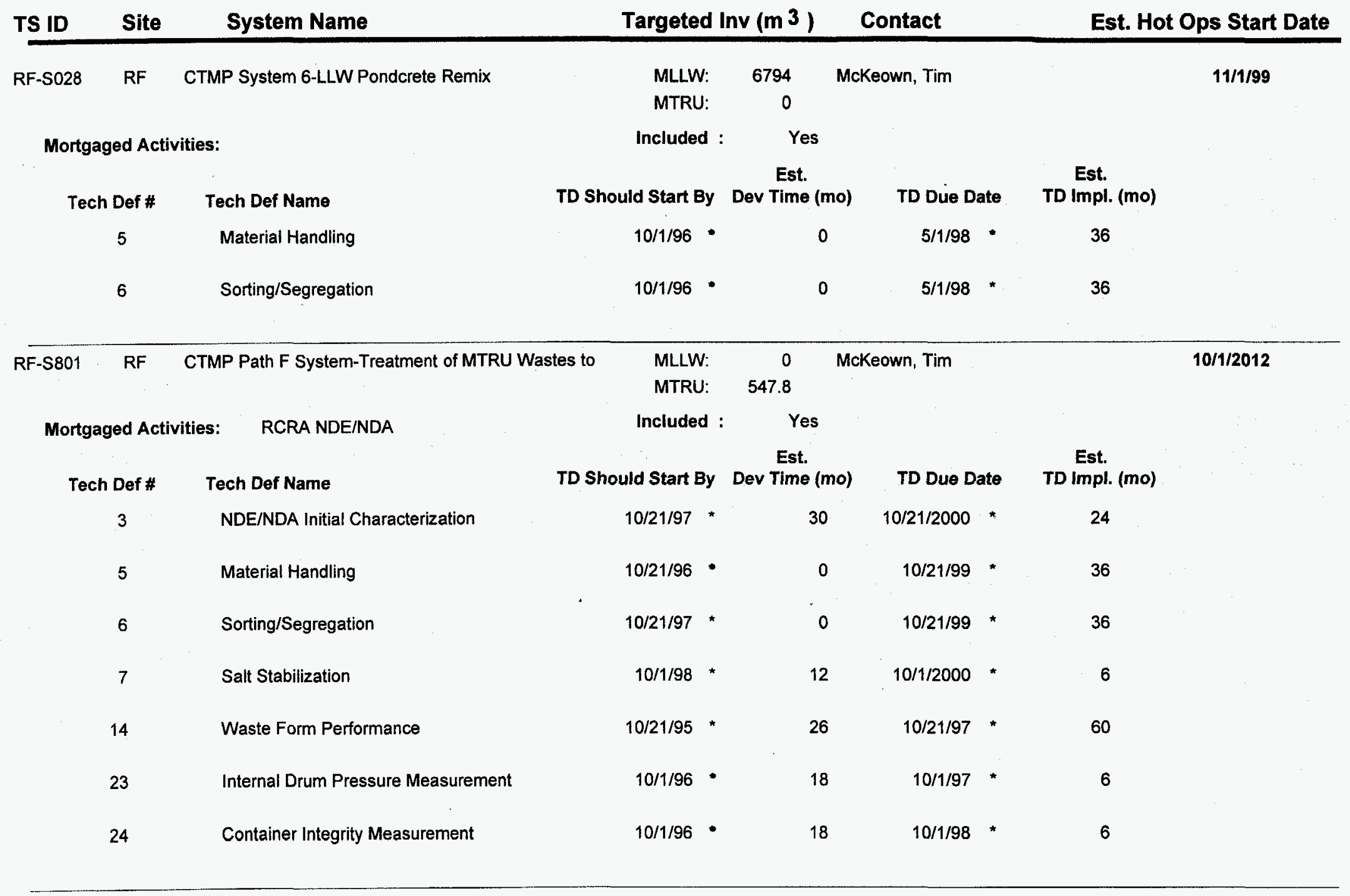




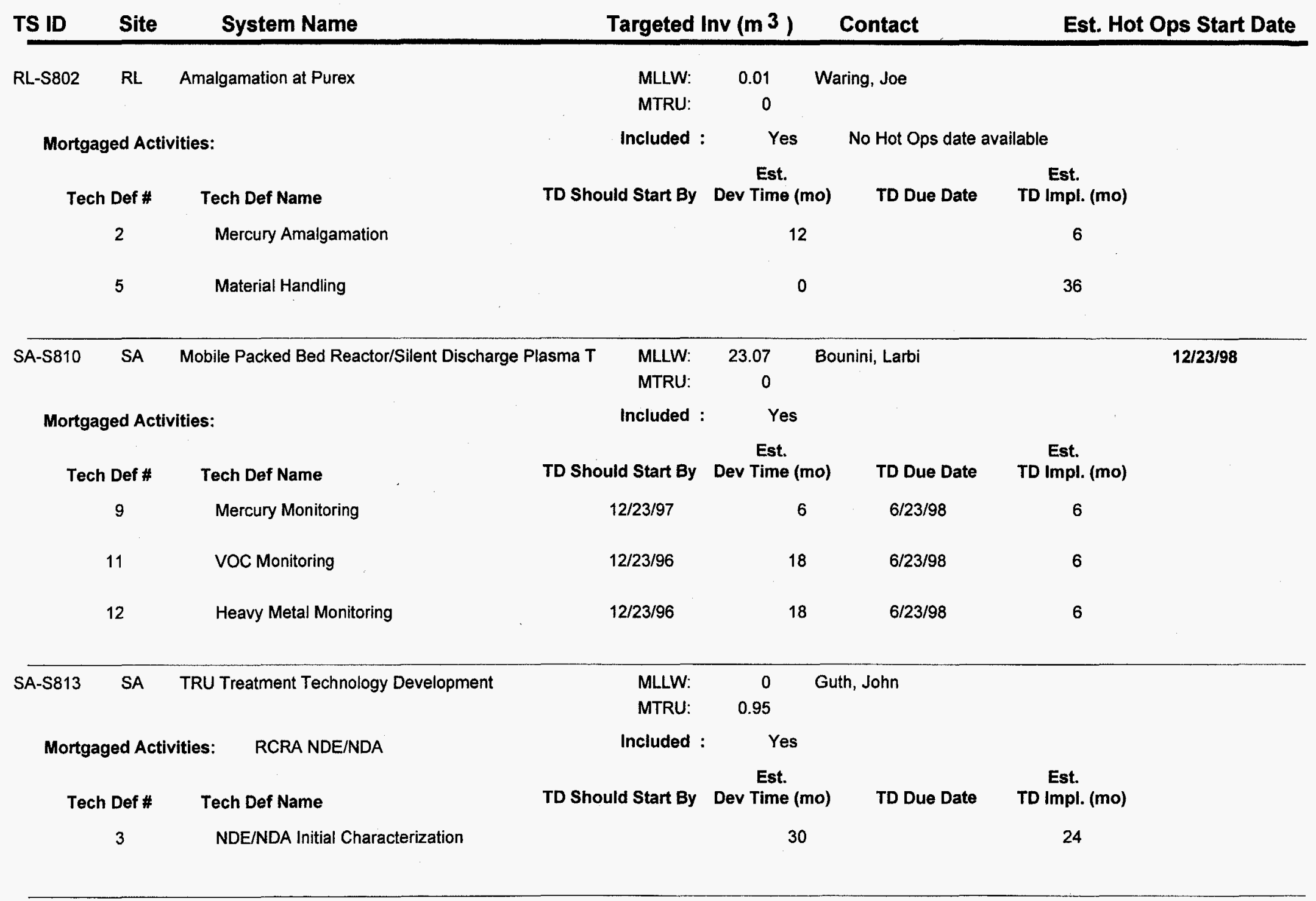




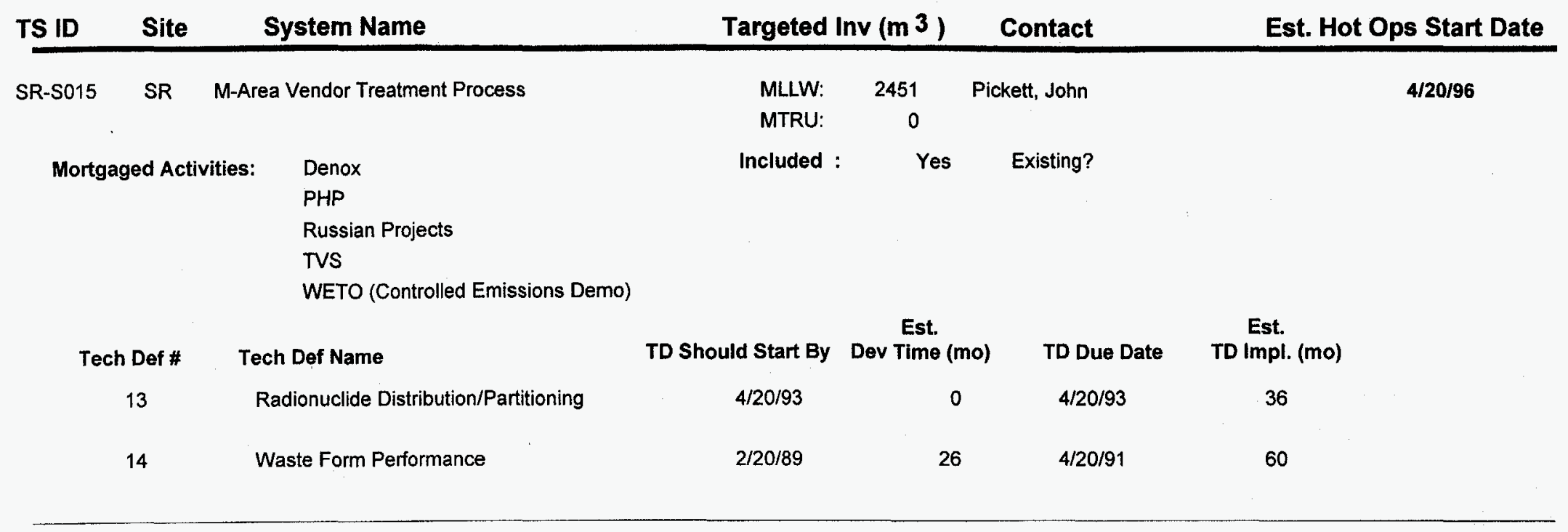




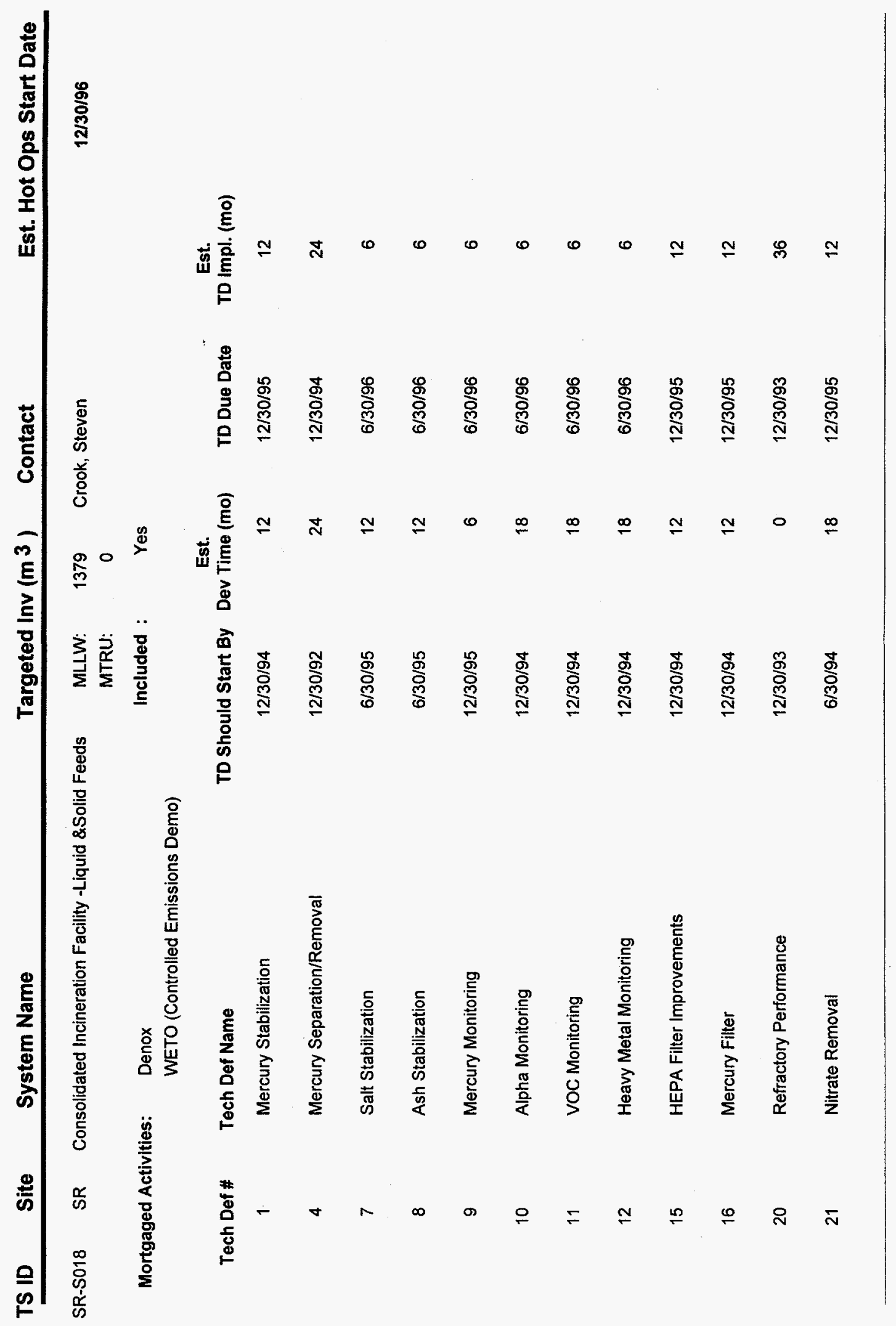

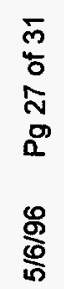




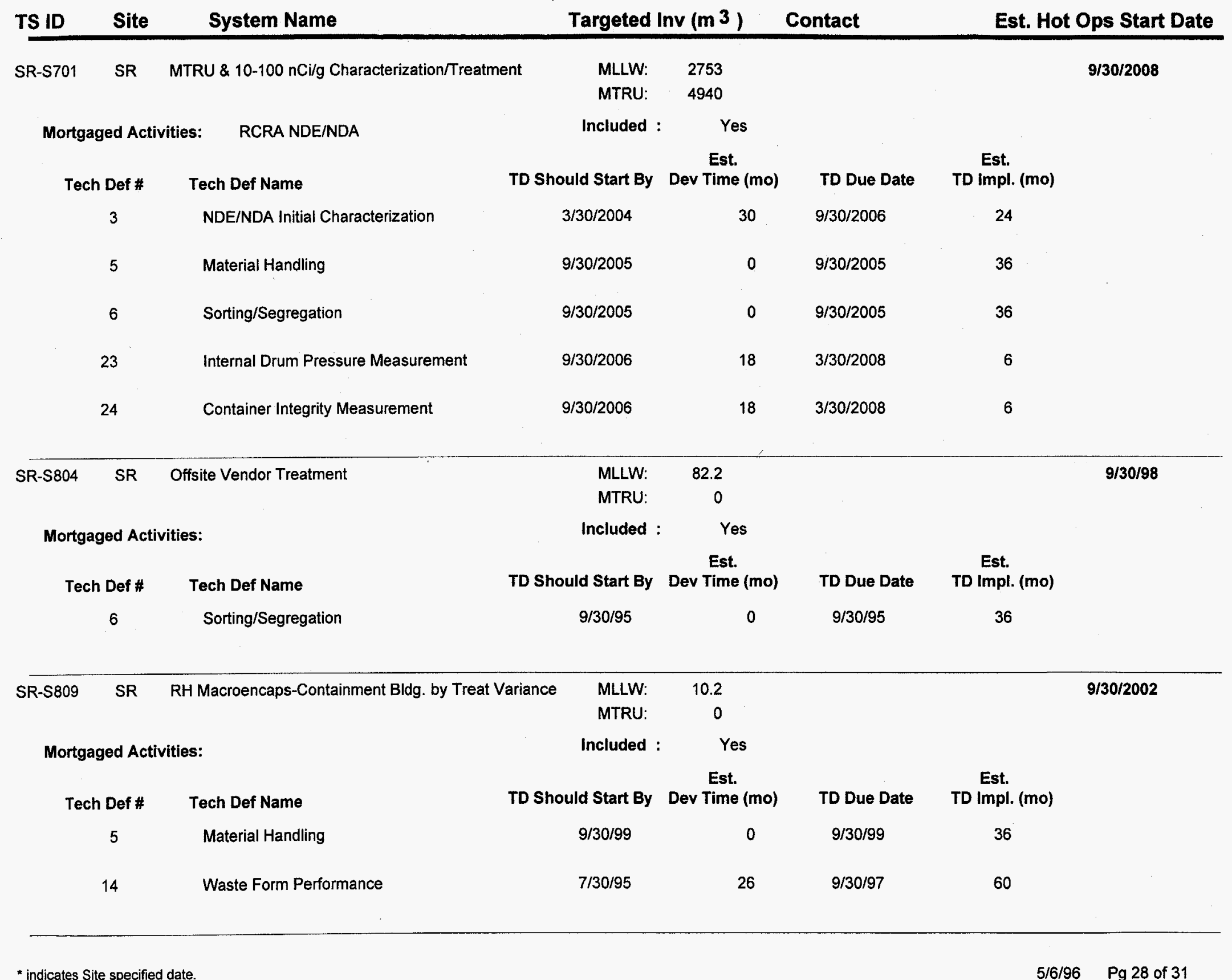




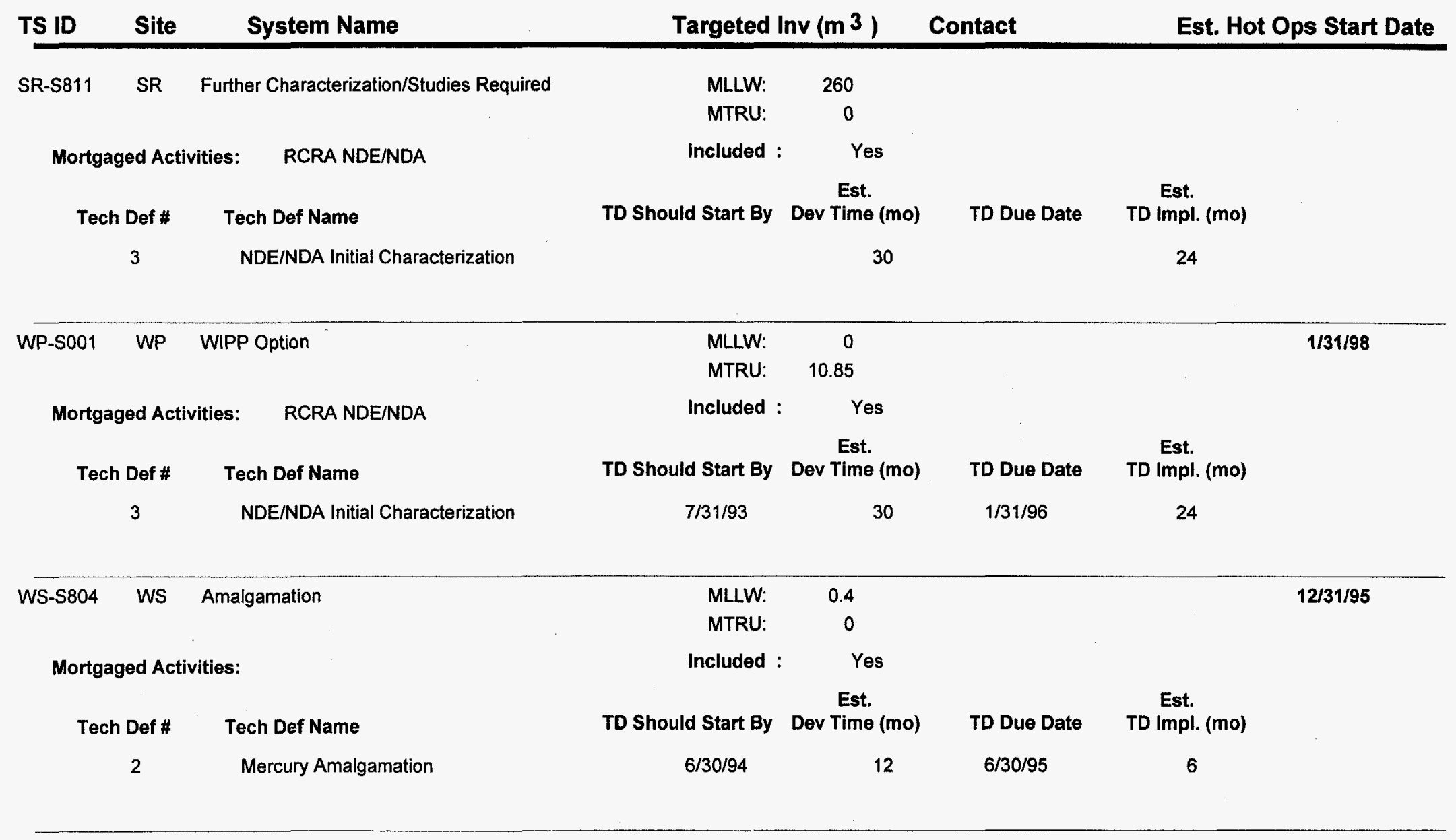




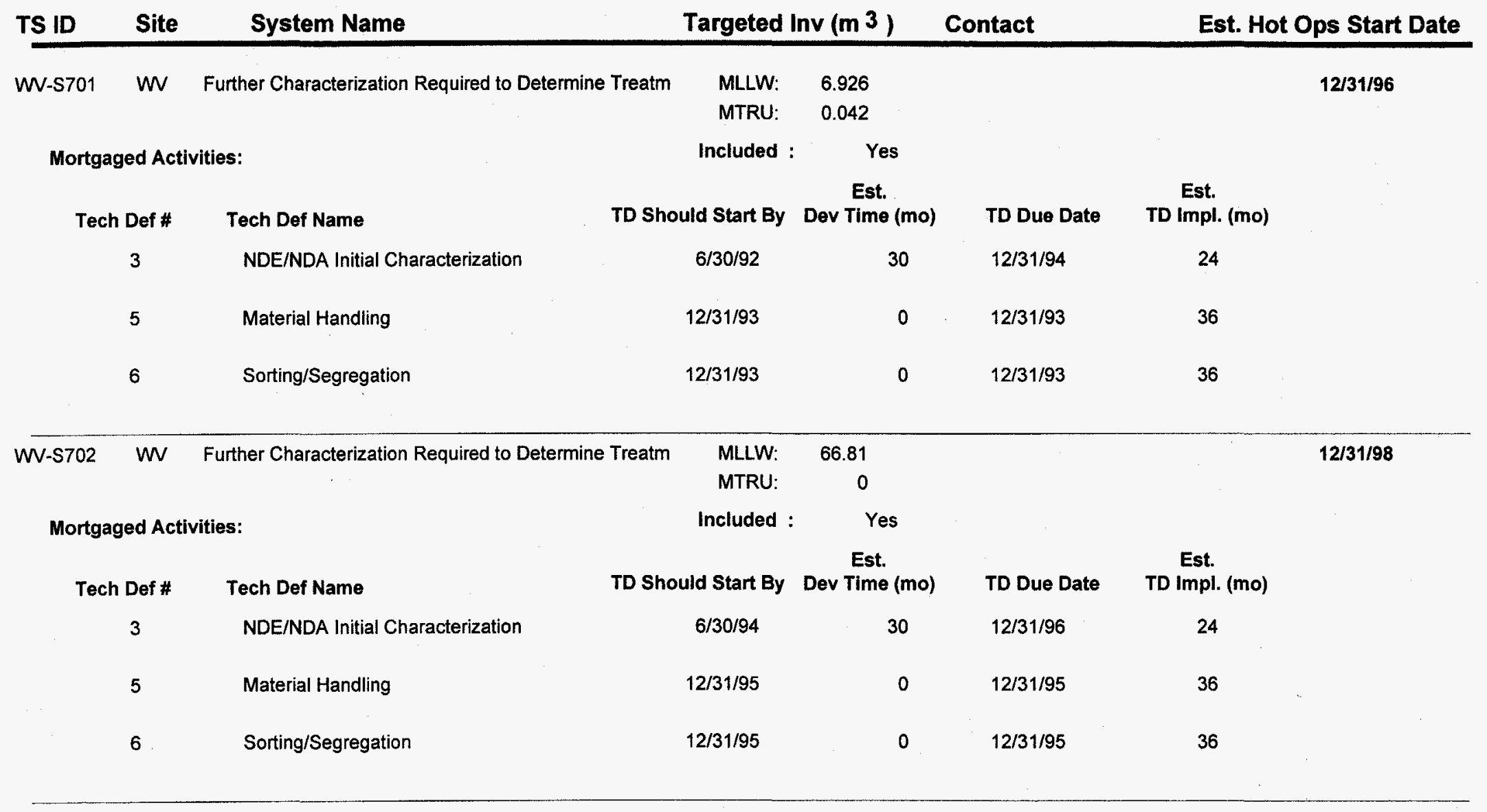




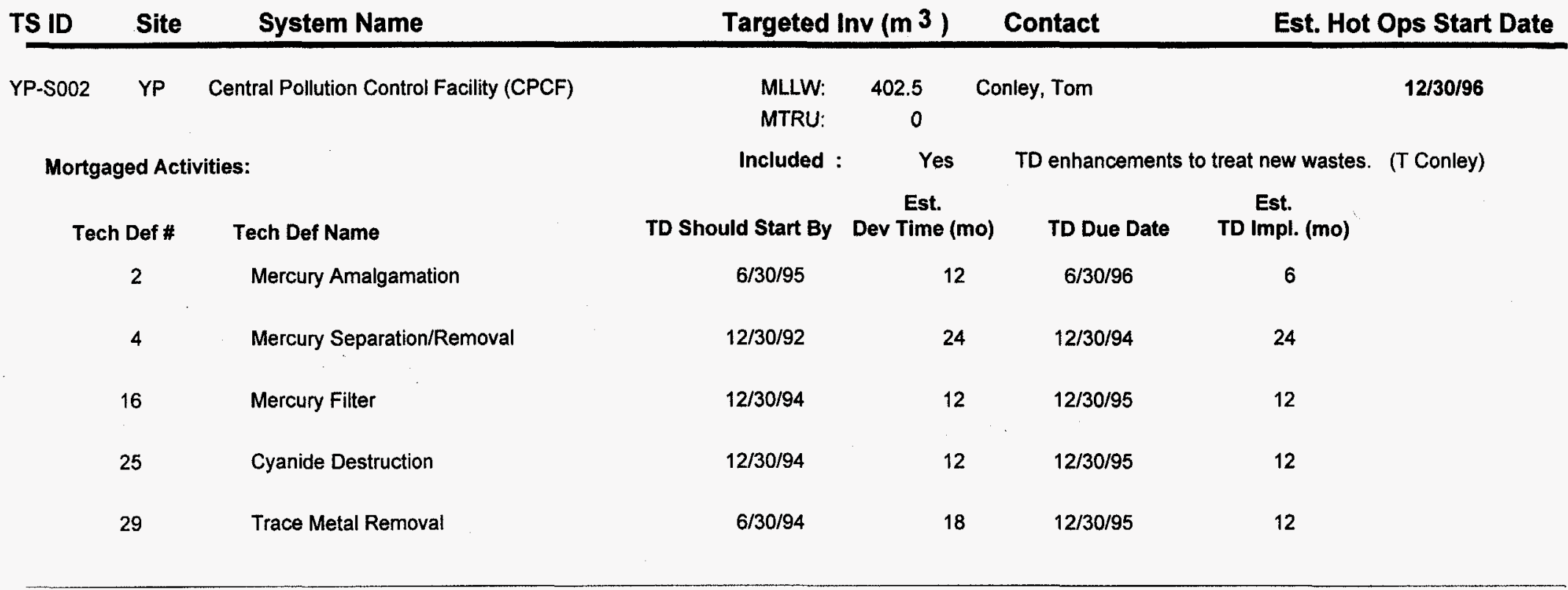


TABLE 2.

\section{IMS - Treatment System by TD Category}

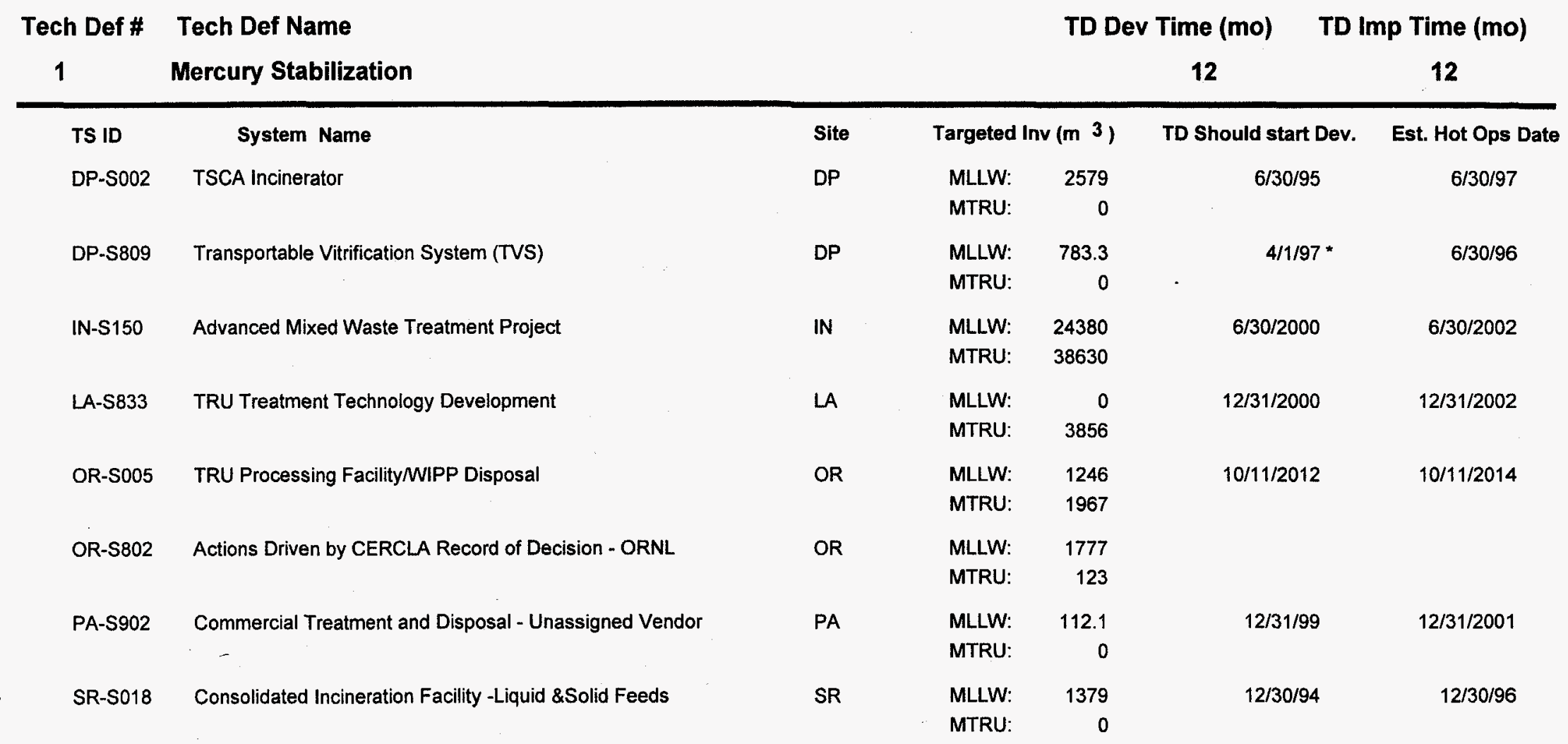




\section{Tech Def \# Tech Def Name}

TD Dev Time (mo) TD Imp Time (mo)

\section{2 Mercury Amalgamation}

TS ID System Name

DP-S002 TSCA Incinerator

DP-S809 . Transportable Vitrification System (TVS)

IN-S150 Advanced Mixed Waste Treatment Project

PI-S801 Mobile Amalgamation Process (Bench Scale)

RL-S802 Amalgamation at Purex

WS-S804 Amalgamation

YP-S002 Central Pollution Control Facility (CPCF)

12

Site

DP

DP

IN

Pl

RL

WS

YP
Targeted Inv ( $\mathbf{m}^{3}$ )

MLLW: 2579

MTRU:

MLLW:

MTRU:

MLLW:

MTRU:

MLLW: MTRU:

MLLW: MTRU:

MLLW: MTRU:

MLLW: MTRU:

24380

38630

0.519

0

0.01

0.4

0

402.5

0
TD Should start Dev.

$$
12 / 30 / 95
$$

$4 / 1 / 97$ *

$12 / 30 / 2000$

$12 / 5 / 98$

$6 / 30 / 94$

6/30/95
6

Est. Hot Ops Date

6/30/97

$6 / 30 / 96$

$6 / 30 / 2002$

$6 / 5 / 2000$

$12 / 31 / 95$

$12 / 30 / 96$ 


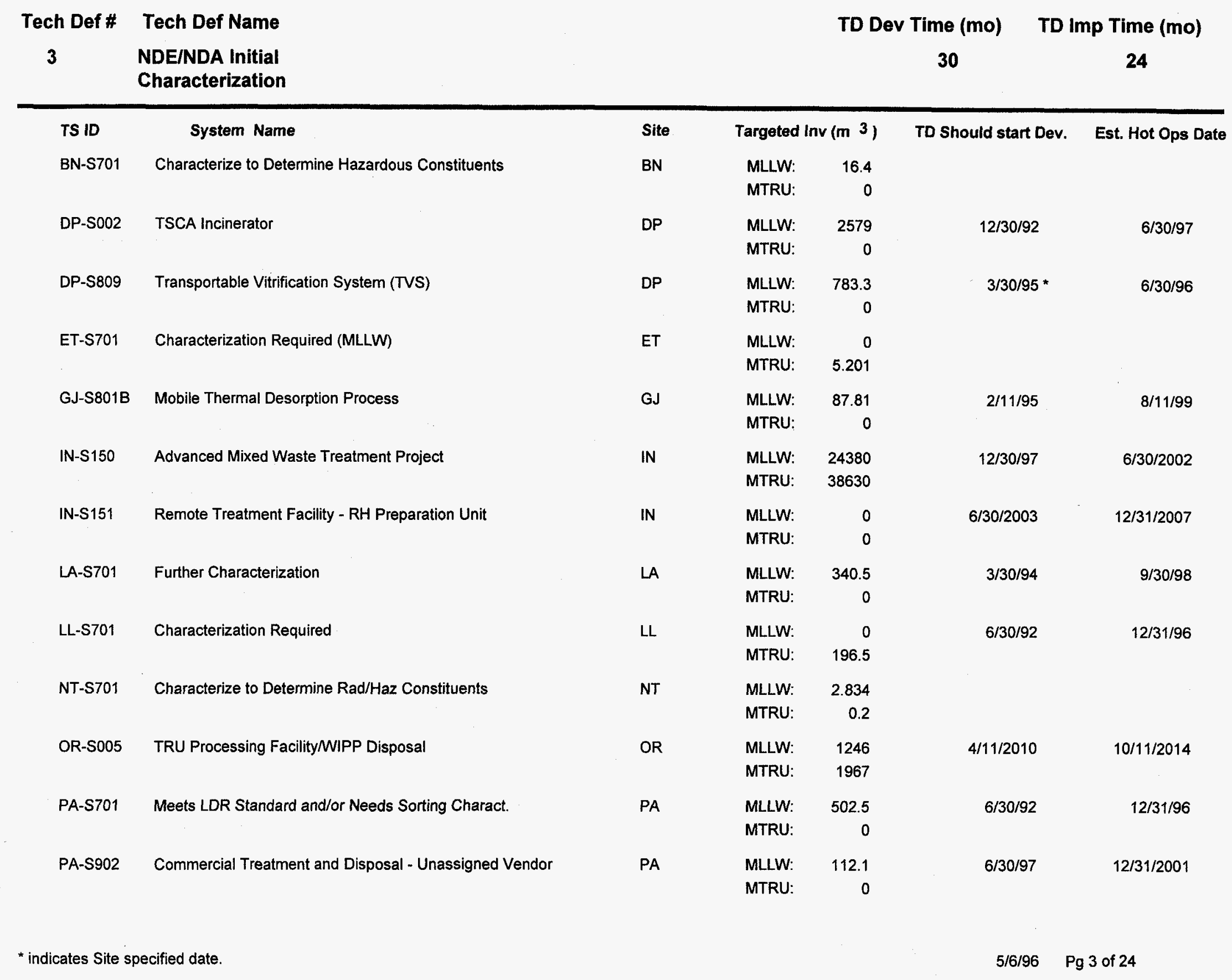




\section{Tech Def \# Tech Def Name}

CTMP Path F System-Treatment of MTRU Wastes to Metw

SA-S813 TRU Treatment Technology Development

SR-S701 MTRU \& 10-100 nCi/g Characterization/Treatment

SR-S811 Further Characterization/Studies Required

WP-S001 WIPP Option

WV-S701 Further Characterization Required to Determine Treatmen

WV-S702 Further Characterization Required to Determine Treatmen
SA

SR

SR

WP

WV

w
TD Dev Time (mo) TD Imp Time (mo)

$4 / 21 / 98$ * $\quad 10 / 1 / 2012$

$\begin{array}{lr}\text { MLLW: } & 0 \\ \text { MTRU: } & 547.8\end{array}$

MLLW: $\quad 0$

MTRU: $\quad 0.95$

MLLW: 2753

MTRU: $\quad 4940$

MLLW: $\quad 260$

MTRU:

MLLW: $\quad 0$

MTRU: $\quad 10.85$

MLLW: $\quad 6.926$

MTRU: $\quad 0.042$

MLLW: $\quad 66.81$

MTRU:
66.81

$7 / 31 / 93$

$1 / 31 / 98$

$6 / 30 / 92$

$12 / 31 / 96$

$6 / 30 / 94$

$12 / 31 / 98$ 


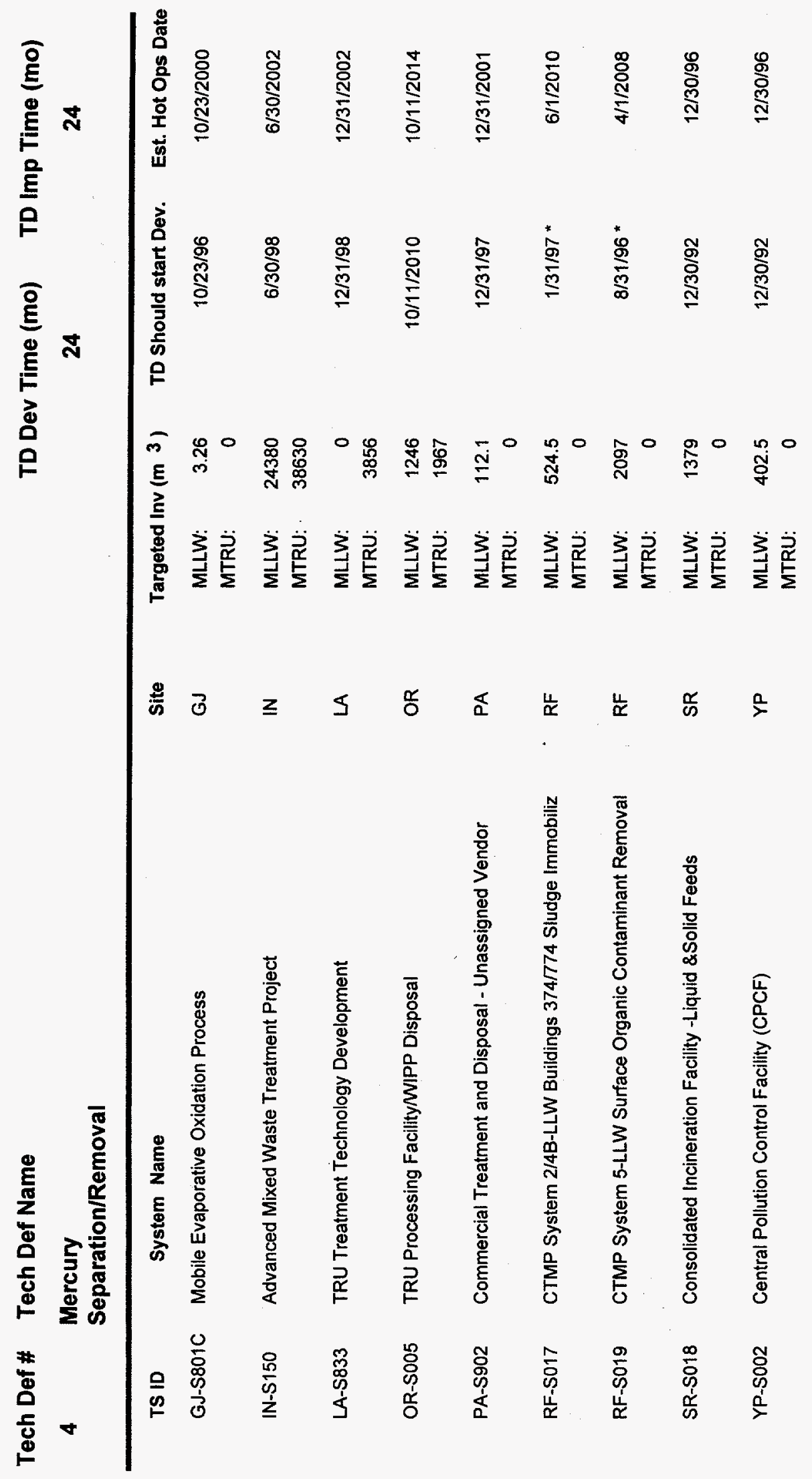

0
0
0
0
0
0 


\begin{tabular}{|c|c|c|c|c|c|c|}
\hline TS ID & System Name & Site & \multicolumn{2}{|c|}{ Targeted Inv (m 3 ) } & TD Should start Dev. & Est. Hot Ops Date \\
\hline \multirow[t]{2}{*}{ AW-S007 } & Remote Treatment Facility (RTF) & AW & MLLW: & 44.45 & $9 / 30 / 2004$ & $9 / 30 / 2007$ \\
\hline & & & MTRU: & 13.88 & & \\
\hline \multirow[t]{2}{*}{ IN-S150 } & Advanced Mixed Waste Treatment Project & $\mathbb{N}$ & MLLW: & 24380 & $6 / 30 / 99$ & $6 / 30 / 2002$ \\
\hline & & & MTRU: & 38630 & & \\
\hline \multirow[t]{2}{*}{ IN-S151 } & Remote Treatment Facility - RH Preparation Unit & IN & MLLW: & 0 & $12 / 31 / 2004$ & $12 / 31 / 2007$ \\
\hline & & & MTRU: & 0 & & \\
\hline \multirow[t]{2}{*}{ IN-S152 } & ICPP - RH Immobilization Facility & IN & MLLW: & 497.9 & $10 / 1 / 2016$ & $10 / 1 / 2019$ \\
\hline & & & MTRU: & 438.6 & & \\
\hline \multirow[t]{2}{*}{ LA-S003 } & Mobile Reactive Metals Skid & LA & MLLW: & 6.83 & $9 / 9 / 2000$ & $9 / 9 / 2003$ \\
\hline & & & MTRU: & 0 & & \\
\hline \multirow[t]{2}{*}{ LA-S701 } & Further Characterization & LA & MLLW: & 340.5 & $9 / 30 / 95$ & $9 / 30 / 98$ \\
\hline & & & MTRU: & 0 & & \\
\hline \multirow[t]{2}{*}{ LA-S833 } & TRU Treatment Technology Development & LA & MLLW: & 0 & $12 / 31 / 99$ & $12 / 31 / 2002$ \\
\hline & & & MTRU: & 3856 & & \\
\hline \multirow[t]{2}{*}{ OR-S005 } & TRU Processing FacilityMIPP Disposal & OR & MLLW: & 1246 & $10 / 11 / 2011$ & $10 / 11 / 2014$ \\
\hline & & & MTRU: & 1967 & & \\
\hline \multirow[t]{2}{*}{ OR-S802 } & Actions Driven by CERCLA Record of Decision - ORNL & OR & MLLW: & 1777 & & \\
\hline & & & MTRU: & 123 & & \\
\hline \multirow[t]{2}{*}{ PO-S806 } & Lab Packs Treatment & $\mathrm{PO}$ & MLLW: & 30.88 & $6 / 30 / 97$ & $6 / 30 / 2000$ \\
\hline & & & MTRU: & 0 & & \\
\hline \multirow[t]{2}{*}{ RF-S019 } & CTMP System 5-LLW Surface Organic Contaminant Removal & RF & MLLW: & 2097 & $8 / 31 / 97^{*}$ & $4 / 1 / 2008$ \\
\hline & & & MTRU: & 0 & & \\
\hline \multirow[t]{2}{*}{ RF-S028 } & CTMP System 6-LLW Pondcrete Remix & RF & MLLW: & 6794 & $5 / 1 / 98^{\bullet}$ & $11 / 1 / 99$ \\
\hline & & & MTRU: & 0 & & \\
\hline \multirow[t]{2}{*}{ RF-\$801 } & CTMP Path F System-Treatment of MTRU Wastes to Meet W & RF & MLLW: & 0 & $10 / 21 / 99 *$ & $10 / 1 / 2012$ \\
\hline & & & MTRU: & 547.8 & & \\
\hline \multirow[t]{2}{*}{ RL-S802 } & Amalgamation at Purex & RL & MLLW: & 0.01 & & \\
\hline & . & & MTRU: & 0 & & \\
\hline ates Site & ified date. & & & & $5 / 6 / 96$ & of 24 \\
\hline
\end{tabular}




\section{Tech Def \# Tech Def Name}

SR-S701 MTRU \& 10-100 nCi/g Characterization/Treatment

SR-S809 RH Macroencaps-Containment Bldg. by Treat Variance

W-S701 Further Characterization Required to Determine Treatmen

W-S702 Further Characterization Required to Determine Treatmen
SR

W

W
TD Dev Time (mo) TD Imp Time (mo)

MLLW: $2753 \quad 9 / 30 / 2005 \quad 9 / 30 / 2008$

MTRU: $\quad 4940$
10.2

MTRU: $\quad 0$

6.926

0.042

66.81

0

9/30/99

$9 / 30 / 2002$

MLLW:

MTRU:
$12 / 31 / 93$

12/31/95 


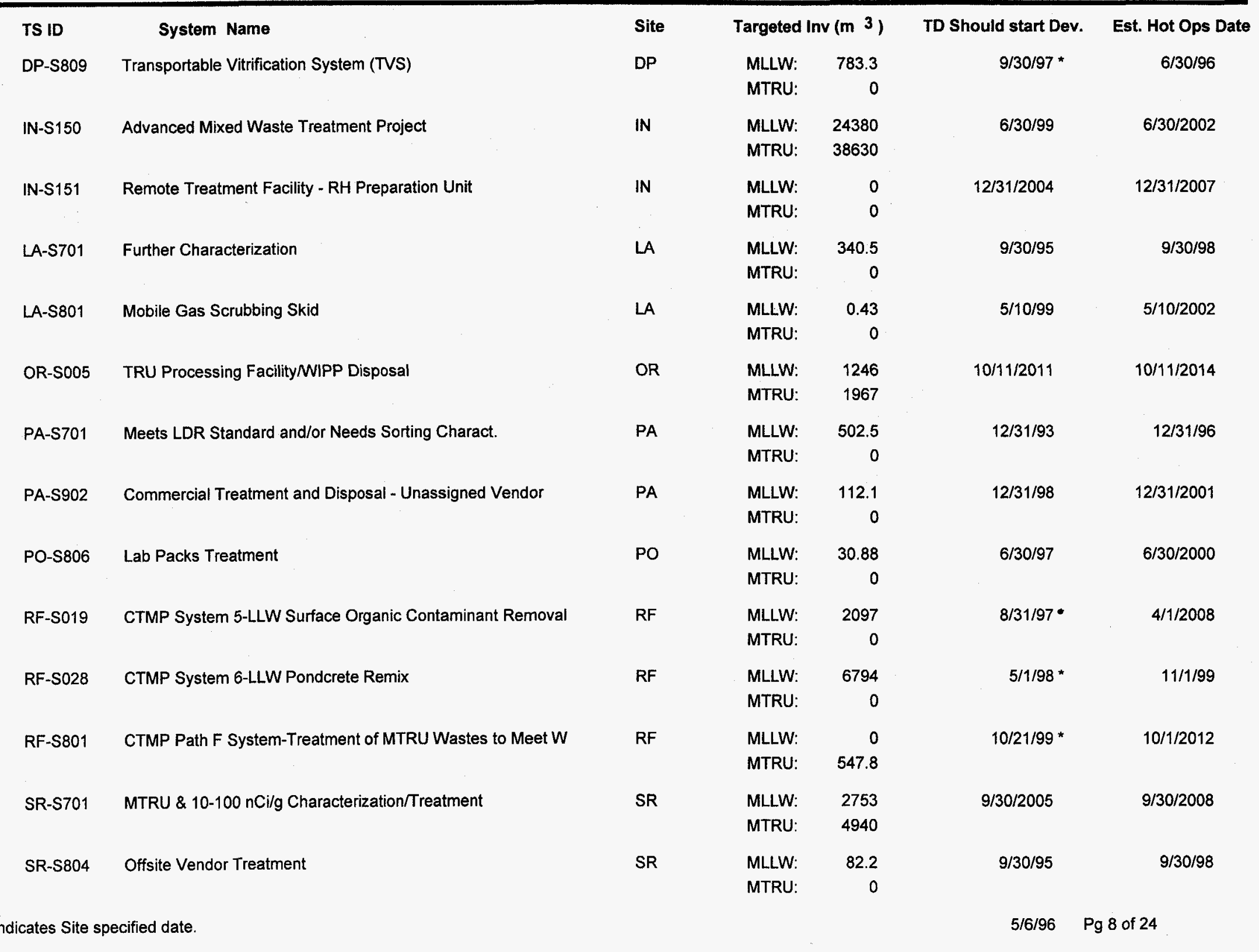



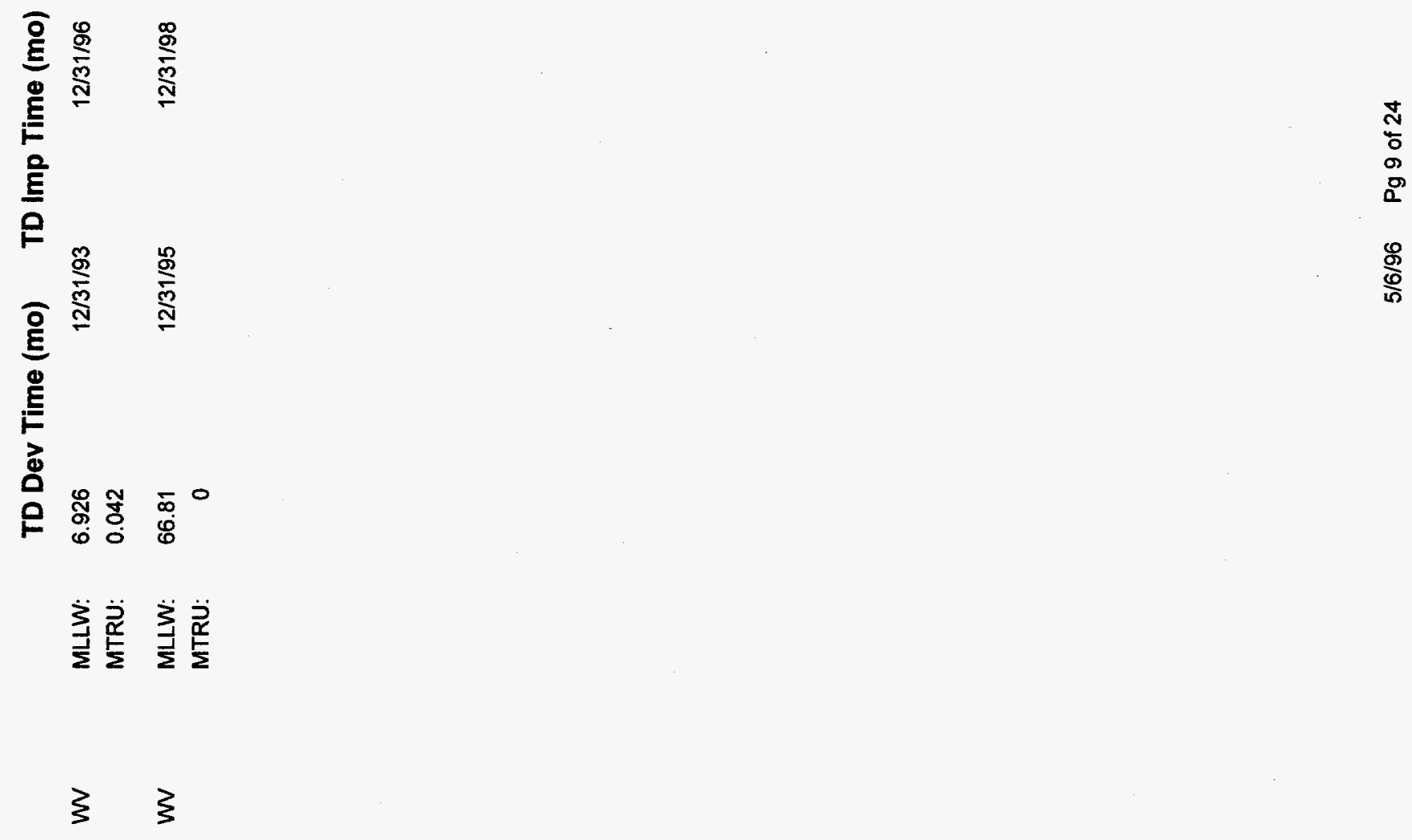

트

号

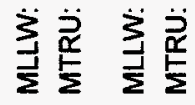

$\xi \quad$

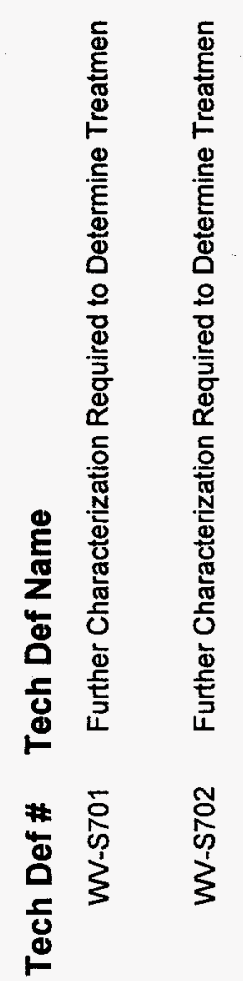




\section{Tech Def \# Tech Def Name}

TD Dev Time (mo) TD Imp Time (mo)

\section{7}

TS ID

IN-S150

System Name

LL-S002

Advanced Mixed Waste Treatment Project

Building 513 Solidification Unit

NT-S703 Treatability Assessment

OR-S005 TRU Processing FacilityMIPP Disposal

PA-\$902

Commercial Treatment and Disposal - Unassigned Vendor

PO-S806

Lab Packs Treatment

RF-S016

CTMP System 3-LLW Miscellaneous Waste Forms Immobiliz

RF-S017

CTMP System 2/4B-LLW Buildings 374/774 Sludge Immobiliz

RF-S019

CTMP System 5-LLW Surface Organic Contaminant Removal

RF-S020

Residue Stabilization

RF-S801

CTMP Path F System-Treatment of MTRU Wastes to Meet W

SR-S018
Consolidated Incineration Facility -Liquid \&Solid Feeds

12

6

Site

IN

LL

NT

OR

PA

PO

RF

$\mathrm{RF}$

RF

RF

RF

SR
Targeted Inv (m 3)

MLLW:

MTRU:

MTRU: $\quad 0$

MLLW: $\quad 285.7$

MTRU: 0

MLLW: 1246

MTRU: 1967

MLLW: 112.1

MTRU: $\quad 0$

MLLW: $\quad 30.88$

MTRU

MLLW

MTRU:

MLLW:

MTRU:

MLLW

MTRU:

MLLW:

MTRU:

MLLW:

MTRU:

MLLW:

MTRU:

0

3501

0

524.5

0

2097

0

0

0.1

0

547.8

1379
0
TD Should start Dev.

$12 / 30 / 2000$

Est. Hot Ops Date

$6 / 30 / 2002$

$4 / 11 / 2013 \quad 10 / 11 / 2014$

$6 / 30 / 2000$

$12 / 31 / 2001$

$12 / 30 / 98 \quad 6 / 30 / 2000$

$9 / 1 / 97$ * $\quad 5 / 31 / 2005$

$7 / 31 / 99$ * $\quad 6 / 1 / 2010$

$10 / 1 / 97^{*} \quad 4 / 1 / 2008$

$6 / 1 / 96$ * $\quad 6 / 1 / 98$

$10 / 1 / 99$ * $\quad 10 / 1 / 2012$

$6 / 30 / 95 \quad 12 / 30 / 96$ 
Tech Def \# Tech Def Name

\begin{tabular}{ll} 
TS ID & \multicolumn{1}{c}{ System Name } \\
DP-S002 & TSCA Incinerator \\
IN-S150 & Advanced Mixed Waste Treatment Project \\
PO-S806 & Lab Packs Treatment \\
RF-S016 & CTMP System 3-LLW Miscellaneous Waste Forms Immob \\
\hline & \\
RF-S020 & Residue Stabilization \\
& \\
SR-S018 & Consolidated Incineration Facility -Liquid \&Solid Feeds
\end{tabular}

\section{Site}

DP

PO

RF

RF

SR
Targeted Inv (m 3 )

MLLW:

MTRU:

2579

MLLW: 24380 MTRU: $\quad 38630$

MLLW: $\quad 30.88$ MTRU:

MLLW: MTRU:

MLLW: MTRU:

MLLW: MTRU:

1379
TD Should start Dev.

$$
12 / 30 / 95
$$

$12 / 30 / 2000$

$12 / 30 / 98$

$9 / 1 / 97^{*}$

$12 / 1 / 97$ *

$6 / 30 / 95$
Est. Hot Ops Date

$6 / 30 / 97$

$6 / 30 / 2002$

$6 / 30 / 2000$

$5 / 31 / 2005$

$6 / 1 / 98$

$12 / 30 / 96$ 
Tech Def \# Tech Def Name

\begin{tabular}{|c|c|c|c|c|c|c|c|}
\hline \multirow{2}{*}{$\begin{array}{l}\text { TS ID } \\
\text { DP-S002 }\end{array}$} & \multirow{2}{*}{$\begin{array}{l}\text { System Name } \\
\text { TSCA Incinerator }\end{array}$} & \multirow{2}{*}{$\begin{array}{l}\text { Site } \\
\text { DP }\end{array}$} & & \multicolumn{2}{|c|}{ Targeted Inv (m ${ }^{3}$ ) } & \multirow{2}{*}{$\begin{array}{c}\text { TD Should start Dev. } \\
\qquad 6 / 30 / 96\end{array}$} & \multirow{2}{*}{$\begin{array}{c}\text { Est. Hot Ops Date } \\
\qquad 6 / 30 / 97\end{array}$} \\
\hline & & & & MLLW: & 2579 & & \\
\hline & & & & MTRU: & 0 & & \\
\hline \multirow[t]{2}{*}{ DP-S809 } & Transportable Vitrification System (TVS) & DP & & MLLW: & 783.3 & $10 / 1 / 97^{*}$ & $6 / 30 / 96$ \\
\hline & & & & MTRU: & 0 & & \\
\hline \multirow[t]{2}{*}{ GJ-S801B } & Mobile Thermal Desorption Process & GJ & & MLLW: & 87.81 & $8 / 11 / 98$ & $8 / 11 / 99$ \\
\hline & & & & MTRU: & 0 & & \\
\hline \multirow[t]{2}{*}{ IN-S005 } & WERF: Incineration - Controlled Air Incinerator & IN & & MLLW: & 115.7 & $9 / 20 / 94$ & 9/20/95 \\
\hline & & & & MTRU: & 0 & & \\
\hline \multirow[t]{2}{*}{ IN-S150 } & Advanced Mixed Waste Treatment Project & IN & & MLLW: & 24380 & $6 / 30 / 2001$ & $6 / 30 / 2002$ \\
\hline & & & 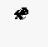 & MTRU: & 38630 & & \\
\hline \multirow[t]{2}{*}{ LA-S833 } & TRU Treatment Technology Development & LA & & MLLW: & 0 & $12 / 30 / 2001$ & $12 / 31 / 2002$ \\
\hline & & & & MTRU: & 3856 & & \\
\hline \multirow[t]{2}{*}{ PO-S806 } & Lab Packs Treatment & $\mathrm{PO}$ & & MLLW: & 30.88 & $6 / 30 / 99$ & $6 / 30 / 2000$ \\
\hline & & & & MTRU: & 0 & & \\
\hline \multirow[t]{2}{*}{ SA-S810 } & Mobile Packed Bed Reactor/Silent Discharge Plasma Treat & SA & & MLLW: & 23.07 & $12 / 23 / 97$ & $12 / 23 / 98$ \\
\hline & & & & MTRU: & 0 & & \\
\hline \multirow[t]{2}{*}{ SR-S018 } & Consolidated Incineration Facility -Liquid \&Solid Feeds & SR & & MLLW: & 1379 & $12 / 30 / 95$ & $12 / 30 / 96$ \\
\hline & & & & MTRU: & 0 & & \\
\hline
\end{tabular}


Tech Def \# Tech Def Name

\begin{tabular}{ll} 
TS ID & \multicolumn{1}{c}{ System Name } \\
DP-S002 & TSCA Incinerator \\
GJ-S801B & Mobile Thermal Desorption Process \\
IN-S150 & Advanced Mixed Waste Treatment Project \\
IN-S152 & ICPP - RH Immobilization Facility \\
& \\
LA-S833 & TRU Treatment Technology Development \\
& \\
PO-S802 & Pretreatment/Regeneration \\
& \\
PO-S806 & Lab Packs Treatment \\
\hline SR-S018 & Consolidated Incineration Facility - Liquid \&Solid Feeds
\end{tabular}

Site

DP

GJ

IN

IN

PO

PO

SR
Targeted Inv ( $\mathrm{m}^{3}$ )

MLLW:

MTRU:

MLLW:

MTRU:

MLLW: 24380

MTRU: $\quad 38630$

MLLW: $\quad 497.9$

MTRU:

438.6

MLLW: 0

MTRU: 3856

MLLW: $\quad 135.5$

MTRU:

MLLW:

MTRU:

30.88

MLLW:

MTRU:
TD Should start Dev. Est. Hot Ops Date

$6 / 30 / 95 \quad 6 / 30 / 97$

$8 / 11 / 97$

$8 / 11 / 99$

$6 / 30 / 2000$

$6 / 30 / 2002$

10/1/2017

$10 / 1 / 2019$

$12 / 30 / 2000$

$12 / 31 / 2002$

$12 / 30 / 99$

$12 / 31 / 2001$

$6 / 30 / 98$

$6 / 30 / 2000$

$12 / 30 / 94$

$12 / 30 / 96$ 


\begin{tabular}{|c|c|c|c|c|c|c|}
\hline $\begin{array}{c}\text { Tech Def \# } \\
11\end{array}$ & $\begin{array}{l}\text { Tech Def Name } \\
\text { voc Monitoring }\end{array}$ & & & TD De & $\begin{array}{l}\text { Time (mo) TD I } \\
18\end{array}$ & $\begin{array}{c}\text { ip Time (mo) } \\
6\end{array}$ \\
\hline TS ID & System Name & Site & Targeted I & $\left(m^{3}\right)$ & TD Should start Dev. & Est. Hot Ops Date \\
\hline DP-S002 & TSCA Incinerator & DP & $\begin{array}{l}\text { MLLW: } \\
\text { MTRU: }\end{array}$ & $\begin{array}{r}2579 \\
0\end{array}$ & $6 / 30 / 95$ & $6 / 30 / 97$ \\
\hline DP-S809 & Transportable Vitrification System (TVS) & $\mathrm{DP}$ & $\begin{array}{l}\text { MLLW: } \\
\text { MTRU: }\end{array}$ & $\begin{array}{r}783.3 \\
0\end{array}$ & 10/1/96 * & $6 / 30 / 96$ \\
\hline DP-S816 & Oak Ridge Commercial Treatment - Sludges & $\mathrm{DP}$ & $\begin{array}{l}\text { MLLW: } \\
\text { MTRU: }\end{array}$ & $\begin{array}{r}6964 \\
0\end{array}$ & $9 / 30 / 94$ & 9/30/96 \\
\hline GJ-S801B & Mobile Thermal Desorption Process & GJ & $\begin{array}{l}\text { MLLW: } \\
\text { MTRU: }\end{array}$ & $\begin{array}{r}87.81 \\
0\end{array}$ & $8 / 11 / 97$ & $8 / 11 / 99$ \\
\hline GJ-S801C & Mobile Evaporative Oxidation Process & GJ & $\begin{array}{l}\text { MLLW: } \\
\text { MTRU: }\end{array}$ & $\begin{array}{r}3.26 \\
0\end{array}$ & $10 / 23 / 98$ & $10 / 23 / 2000$ \\
\hline IN-S005 & WERF: Incineration - Controlled Air Incinerator & IN & $\begin{array}{l}\text { MLLW: } \\
\text { MTRU: }\end{array}$ & $\begin{array}{r}115.7 \\
0\end{array}$ & $9 / 20 / 93$ & 9/20/95 \\
\hline IN-S150 & Advanced Mixed Waste Treatment Project & IN & $\begin{array}{l}\text { MLLW: } \\
\text { MTRU: }\end{array}$ & $\begin{array}{l}24380 \\
38630\end{array}$ & $6 / 30 / 2000$ & $6 / 30 / 2002$ \\
\hline LA-S833 & TRU Treatment Technology Development & LA & $\begin{array}{l}\text { MLLW: } \\
\text { MTRU: }\end{array}$ & $\begin{array}{r}0 \\
3856\end{array}$ & $12 / 30 / 2000$ & 12/31/2002 \\
\hline PO-S802 & PretreatmentRegeneration & PO & $\begin{array}{l}\text { MLLW: } \\
\text { MTRU: }\end{array}$ & $\begin{array}{r}135.5 \\
0\end{array}$ & $12 / 30 / 99$ & $12 / 31 / 2001$ \\
\hline PO-S806 & Lab Packs Treatment & Po & $\begin{array}{l}\text { MLLW: } \\
\text { MTRU: }\end{array}$ & $\begin{array}{r}30.88 \\
0\end{array}$ & $6 / 30 / 98$ & $6 / 30 / 2000$ \\
\hline RF-S019 & CTMP System 5-LLW Surface Organic Contaminant Removal & RF & $\begin{array}{l}\text { MLLW: } \\
\text { MTRU: }\end{array}$ & $\begin{array}{r}2097 \\
0\end{array}$ & $4 / 1 / 97$ * & $4 / 1 / 2008$ \\
\hline SA-S810 & Mobile Packed Bed Reactor/Silent Discharge Plasma Treat & SA & $\begin{array}{l}\text { MLLW: } \\
\text { MTRU: }\end{array}$ & $\begin{array}{r}23.07 \\
0\end{array}$ & $12 / 23 / 96$ & $12 / 23 / 98$ \\
\hline SR-S018 & Consolidated Incineration Facility -Liquid \&Solid Feeds & SR & $\begin{array}{l}\text { MLLW: } \\
\text { MTRU: }\end{array}$ & $\begin{array}{r}1379 \\
0\end{array}$ & $12 / 30 / 94$ & $12 / 30 / 96$ \\
\hline
\end{tabular}




\begin{tabular}{|c|c|c|c|c|c|c|}
\hline $\begin{array}{l}\text { Tech Def \# } \\
12\end{array}$ & $\begin{array}{l}\text { Tech Def Name } \\
\text { Heavy Metal Monitoring }\end{array}$ & & & TD D & $\begin{array}{c}\text { Time (mo) } \\
18\end{array}$ & $\begin{array}{c}\text { lp Time (mo) } \\
6\end{array}$ \\
\hline TS ID & System Name & Site & Targeted & $v\left(\mathbf{m}^{3}\right)$ & TD Should start Dev. & Est. Hot Ops Date \\
\hline DP-S002 & TSCA Incinerator & DP & $\begin{array}{l}\text { MLLW: } \\
\text { MTRU: }\end{array}$ & $\begin{array}{r}2579 \\
0\end{array}$ & $6 / 30 / 95$ & $6 / 30 / 97$ \\
\hline DP-S809 & Transportable Vitrification System (TVS) & $\mathrm{DP}$ & $\begin{array}{l}\text { MLLW: } \\
\text { MTRU: }\end{array}$ & $\begin{array}{r}783.3 \\
0\end{array}$ & $10 / 1 / 96 *$ & $6 / 30 / 96$ \\
\hline IN-S005 & WERF: Incineration - Controlled Air Incinerator & $\mathbb{I N}$ & $\begin{array}{l}\text { MLLW: } \\
\text { MTRU: }\end{array}$ & $\begin{array}{r}115.7 \\
0\end{array}$ & 9/20/93 & 9/20/95 \\
\hline IN-S150 & Advanced Mixed Waste Treatment Project & $\mathbb{I N}$ & $\begin{array}{l}\text { MLLW: } \\
\text { MTRU: }\end{array}$ & $\begin{array}{l}24380 \\
38630\end{array}$ & $6 / 30 / 2000$ & $6 / 30 / 2002$ \\
\hline IN-S152 & ICPP - RH Immobilization Facility & $\mathbb{I N}$ & $\begin{array}{l}\text { MLLW: } \\
\text { MTRU: }\end{array}$ & $\begin{array}{l}497.9 \\
438.6\end{array}$ & $10 / 1 / 2017$ & $10 / 1 / 2019$ \\
\hline LA-S833 & TRU Treatment Technology Development & LA & $\begin{array}{l}\text { MLLW: } \\
\text { MTRU: }\end{array}$ & $\begin{array}{r}0 \\
3856\end{array}$ & $12 / 30 / 2000$ & $12 / 31 / 2002$ \\
\hline PO-S802 & Pretreatment/Regeneration & Po & $\begin{array}{l}\text { MLLW: } \\
\text { MTRU: }\end{array}$ & $\begin{array}{r}135.5 \\
0\end{array}$ & $12 / 30 / 99$ & $12 / 31 / 2001$ \\
\hline PO-S806 & Lab Packs Treatment & PO & $\begin{array}{l}\text { MLLW: } \\
\text { MTRU: }\end{array}$ & $\begin{array}{r}30.88 \\
0\end{array}$ & $6 / 30 / 98$ & $6 / 30 / 2000$ \\
\hline SA-S810 & Mobile Packed Bed iReactor/Silent Discharge Plasma Treat & SA & $\begin{array}{l}\text { MLLW: } \\
\text { MTRU: }\end{array}$ & $\begin{array}{r}23.07 \\
0\end{array}$ & 12/23/96 & $12 / 23 / 98$ \\
\hline SR-S018 & Consolidated Incineration Facility -Liquid \&Solid Feeds & SR & $\begin{array}{l}\text { MLLW: } \\
\text { MTRU: }\end{array}$ & $\begin{array}{r}1379 \\
0\end{array}$ & $12 / 30 / 94$ & $12 / 30 / 96$ \\
\hline
\end{tabular}


Tech Def \# Tech Def Name

TD Dev Time (mo)

o

TD Imp Time (mo)

13 Radionuclide

Distribution/Partitioning

TS ID

System Name

DP-S816 Oak Ridge Commercial Treatment - Sludges

IN-S150 Advanced Mixed Waste Treatment Project

OR-S005 TRU Processing FacilityMIPP Disposal

SR-S015 M-Area Vendor Treatment Process
Site

IN

OR

SR
Targeted Inv (m 3 )

MLLW

MTRU:

MLLW: $\quad 24380$

MTRU:

MLLW:

MTRU:

MLLW:

MTRU:
36
TD Should start Dev. Est. Hot Ops Date

$\begin{array}{ll}9 / 30 / 93 & 9 / 30 / 96\end{array}$

$\begin{array}{lll}64380 & 6 / 30 / 99 & 6 / 30 / 2002\end{array}$

$1246 \quad 10 / 11 / 2011 \quad 10 / 11 / 2014$

$4 / 20 / 93 \quad 4 / 20 / 96$ 


\begin{tabular}{|c|c|c|c|c|c|c|}
\hline \multirow{2}{*}{$\begin{array}{l}\text { TS ID } \\
\text { DP-S809 }\end{array}$} & \multirow{3}{*}{$\begin{array}{l}\text { System Name } \\
\text { Transportable Vitrification System (TVS) }\end{array}$} & \multirow{3}{*}{$\begin{array}{l}\text { Site } \\
\text { DP }\end{array}$} & \multicolumn{2}{|c|}{ Targeted Inv (m 3 ) } & \multirow{2}{*}{$\begin{array}{l}\text { TD Should start Dev. } \\
\qquad 7 / 30 / 95^{*}\end{array}$} & \multirow{3}{*}{$\begin{array}{l}\text { Est. Hot Ops Date } \\
6 / 30 / 96\end{array}$} \\
\hline & & & MLLW: & 783.3 & & \\
\hline & & & MTRU: & 0 & & \\
\hline \multirow{2}{*}{ DP-S816 } & Oak Ridge Commercial Treatment - Sludges & DP & MLLW: & 6964 & $7 / 30 / 89$ & $9 / 30 / 96$ \\
\hline & & & MTRU: & 0 & & \\
\hline \multirow[t]{2}{*}{ IN-S150 } & Advanced Mixed Waste Treatment Project & IN & MLLW: & 24380 & $4 / 30 / 95$ & $6 / 30 / 2002$ \\
\hline & & & MTRU: & 38630 & & \\
\hline \multirow[t]{2}{*}{ IN-S152 } & ICPP - RH Immobilization Facility & IN & MLLW: & 497.9 & $8 / 1 / 2012$ & $10 / 1 / 2019$ \\
\hline & & & MTRU: & 438.6 & & \\
\hline \multirow[t]{2}{*}{ LA-S833 } & TRU Treatment Technology Development & LA & MLLW: & 0 & $10 / 31 / 95$ & $12 / 31 / 2002$ \\
\hline & & & MTRU: & 3856 & & \\
\hline \multirow[t]{2}{*}{ LL-S002 } & Building 513 Solidification Unit & $\mathbf{L L}$ & MLLW: & 160.8 & & \\
\hline & & & MTRU: & 0 & & \\
\hline \multirow[t]{2}{*}{ OR-S005 } & TRU Processing FacilityMIPP Disposal & OR & MLLW: & 1246 & $8 / 11 / 2007$ & $10 / 11 / 2014$ \\
\hline & & & MTRU: & 1967 & & \\
\hline \multirow[t]{2}{*}{ PA-S902 } & Commercial Treatment and Disposal - Unassigned Vendor & PA & MLLW: & 112.1 & $10 / 31 / 94$ & $12 / 31 / 2001$ \\
\hline & & & MTRU: & 0 & & \\
\hline \multirow[t]{2}{*}{ PO-\$806 } & Lab Packs Treatment & PO & MLLW: & 30.88 & $4 / 30 / 93$ & $6 / 30 / 2000$ \\
\hline & & & MTRU: & 0 & & \\
\hline \multirow[t]{2}{*}{ RF-S017 } & CTMP System 2/4B-LLW Buildings 374/774 Sludge Immobiliz & RF & MLLW: & 524.5 & $11 / 30 / 93^{*}$ & $6 / 1 / 2010$ \\
\hline & & & MTRU: & 0 & & \\
\hline \multirow[t]{2}{*}{ RF-S020 } & Residue Stabilization & RF & MLLW: & 0 & $4 / 1 / 91$ & $6 / 1 / 98$ \\
\hline & & & MTRU: & 0.1 & & \\
\hline \multirow[t]{2}{*}{ RF-S801 } & CTMP Path F System-Treatment of MTRU Wastes to Meet W & RF & MLLW: & 0 & $8 / 21 / 95^{\bullet}$ & $10 / 1 / 2012$ \\
\hline & & & MTRU: & 547.8 & & \\
\hline \multirow[t]{2}{*}{ SR-S015 } & M-Area Vendor Treatment Process & SR & MLLW: & 2451 & $2 / 20 / 89$ & $4 / 20 / 96$ \\
\hline & & & MTRU: & 0 & & \\
\hline \multirow[t]{2}{*}{ SR-S809 } & RH Macroencaps-Containment Bldg. by Treat Variance & SR & MLLW: & 10.2 & $7 / 30 / 95$ & $9 / 30 / 2002$ \\
\hline & & & MTRU: & 0 & & \\
\hline tes Site & fied date. & & & & $5 / 6 / 96$ & 7 of 24 \\
\hline
\end{tabular}




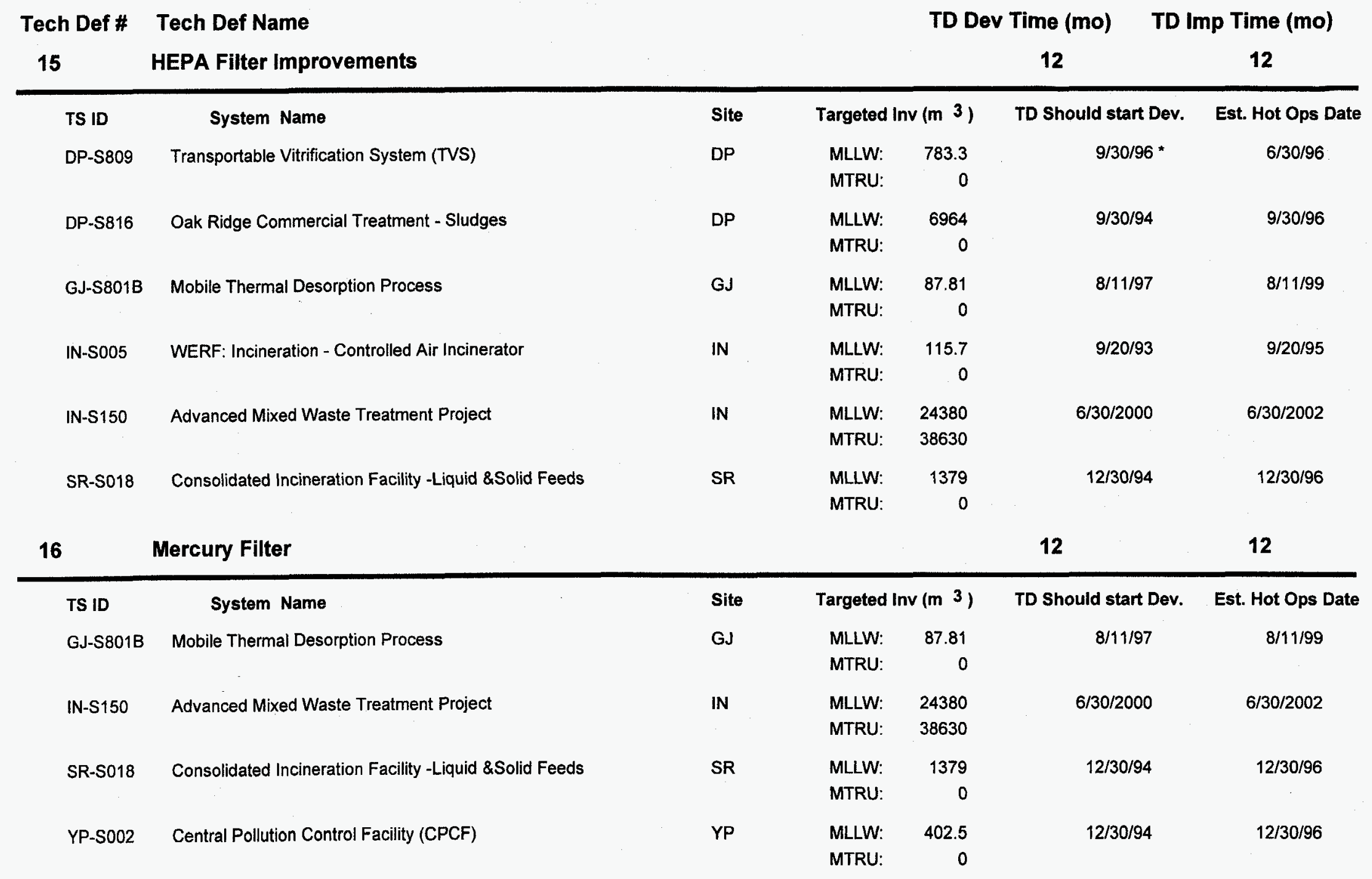




\begin{tabular}{|c|c|c|c|c|c|c|}
\hline $\begin{array}{l}\text { Tech Def \# } \\
17\end{array}$ & $\begin{array}{l}\text { Tech Def Name } \\
\text { Molten Product - Recanting }\end{array}$ & & & TD D & $\begin{array}{c}\text { Time (mo) } \\
0\end{array}$ & $\begin{array}{l}\text { up Time (mo) } \\
\quad 36\end{array}$ \\
\hline TS ID & System Name & Site & Targeted I & $v(m)$ & TD Should start Dev. & Est. Hot Ops Date \\
\hline IN-S150 & Advanced Mixed Waste Treatment Project & $\mathbb{I N}$ & $\begin{array}{l}\text { MLLW: } \\
\text { MTRU: }\end{array}$ & $\begin{array}{l}24380 \\
38630\end{array}$ & $6 / 30 / 99$ & $6 / 30 / 2002$ \\
\hline IN-S152 & ICPP - RH Immobilization Facility & IN & $\begin{array}{l}\text { MLLW: } \\
\text { MTRU: }\end{array}$ & $\begin{array}{l}497.9 \\
438.6\end{array}$ & $10 / 1 / 2016$ & $10 / 1 / 2019$ \\
\hline LA-S833 & TRU Treatment Technology Development & LA & $\begin{array}{l}\text { MLLW: } \\
\text { MTRU: }\end{array}$ & $\begin{array}{r}0 \\
3856\end{array}$ & $12 / 31 / 99$ & $12 / 31 / 2002$ \\
\hline 18 & $\begin{array}{l}\text { Comparative } \\
\text { Analysis/Aqueous }\end{array}$ & & & & 6 & 24 \\
\hline TS ID & System Name & Site & Targeted I & $v\left(m^{3}\right)$ & TD Should start Dev. & Est. Hot Ops Date \\
\hline FM-S801 & Ohio Option-Mobile Chem. Tmt Project & $\mathrm{FM}$ & $\begin{array}{l}\text { MLLW: } \\
\text { MTRU: }\end{array}$ & $\begin{array}{r}650.9 \\
0\end{array}$ & $10 / 30 / 93$ & $4 / 30 / 96$ \\
\hline PO-S806 & Lab Packs Treatment & PO & $\begin{array}{l}\text { MLLW: } \\
\text { MTRU: }\end{array}$ & $\begin{array}{r}30.88 \\
0\end{array}$ & $12 / 30 / 97$ & $6 / 30 / 2000$ \\
\hline PO-S807 & Metal Recovery & PO & $\begin{array}{l}\text { MLLW: } \\
\text { MTRU: }\end{array}$ & $\begin{array}{r}62.25 \\
0\end{array}$ & $3 / 30 / 99$ & $9 / 30 / 2001$ \\
\hline PO-S809 & On-Site Stabilization & Po & $\begin{array}{l}\text { MLLW: } \\
\text { MTRU: }\end{array}$ & $\begin{array}{r}5383 \\
0\end{array}$ & $3 / 30 / 2000$ & $9 / 30 / 2002$ \\
\hline
\end{tabular}


Tech Def \# Tech Def Name

\begin{tabular}{|c|c|c|c|c|c|c|}
\hline TS ID & System Name & Site & \multicolumn{2}{|c|}{ Targeted Inv $\left(\mathrm{m}^{3}\right)$} & TD Should start Dev. & Est. Hot Ops Date \\
\hline \multirow[t]{2}{*}{ FM-S801 } & Ohio Option-Mobile Chem. Tmt Project & $\mathrm{FM}$ & MLLW: & 650.9 & $4 / 30 / 92$ & $4 / 30 / 96$ \\
\hline & & & MTRU: & 0 & & \\
\hline \multirow[t]{2}{*}{ LA-S004 } & Mobile Plating Wastes Acids/Bases Skid & LA & MLLW: & 3.491 & $3 / 17 / 96$ & $3 / 17 / 2000$ \\
\hline & & & MTRU: & 0 & & \\
\hline \multirow[t]{2}{*}{ PO-S806 } & Lab Packs Treatment & $\mathrm{PO}$ & MLLW: & 30.88 & $6 / 30 / 96$ & $6 / 30 / 2000$ \\
\hline & & & MTRU: & 0 & & \\
\hline \multirow[t]{2}{*}{ PO-S807 } & Metal Recovery & $\mathrm{PO}$ & MLLW: & 62.25 & $9 / 30 / 97$ & $9 / 30 / 2001$ \\
\hline & & & MTRU: & 0 & & \\
\hline \multirow[t]{2}{*}{ PO-S809 } & On-Site Stabilization & $\mathrm{PO}$ & MLLW: & 5383 & $9 / 30 / 98$ & $9 / 30 / 2002$ \\
\hline & & & MTRU: & 0 & & \\
\hline \multirow[t]{2}{*}{ RF-S019 } & CTMP System 5-LLW Surface Organic Contaminant Removal & $\mathrm{RF}$ & MLLW: & 2097 & $10 / 1 / 96 *$ & $4 / 1 / 2008$ \\
\hline & & & MTRU: & 0 & & \\
\hline 20 & Refractory Performance & & & & 0 & 36 \\
\hline TS ID & System Name & Site & \multicolumn{2}{|c|}{ Targeted Inv ( $\left.\mathrm{m}^{3}\right)$} & TD Should start Dev. & Est. Hot Ops Date \\
\hline \multirow[t]{2}{*}{ DP-S809 } & Transportable Vitrification System (TVS) & DP & MLLW: & 783.3 & $9 / 30 / 97 *$ & $6 / 30 / 96$ \\
\hline & & & MTRU: & 0 & & \\
\hline \multirow[t]{2}{*}{ DP-S816 } & Oak Ridge Commercial Treatment - Sludges & $\mathrm{DP}$ & MLLW: & 6964 & $9 / 30 / 93$ & $9 / 30 / 96$ \\
\hline & & & MTRU: & 0 & & \\
\hline \multirow[t]{2}{*}{ IN-S150 } & Advanced Mixed Waste Treatment Project & IN & MLLW: & 24380 & $6 / 30 / 99$ & $6 / 30 / 2002$ \\
\hline & & & MTRU: & 38630 & & \\
\hline \multirow[t]{2}{*}{ IN-S152 } & ICPP - RH Immobilization Facility & IN & MLLW: & 497.9 & $10 / 1 / 2016$ & $10 / 1 / 2019$ \\
\hline & & & MTRU: & 438.6 & & \\
\hline \multirow[t]{2}{*}{ LA-S833 } & TRU Treatment Technology Development & LA & MLLW: & 0 & $12 / 31 / 99$ & $12 / 31 / 2002$ \\
\hline & & & MTRU: & 3856 & & \\
\hline \multirow[t]{2}{*}{ SR-S018 } & Consolidated Incineration Facility -Liquid \&Solid Feeds & SR & MLLW: & 1379 & $12 / 30 / 93$ & $12 / 30 / 96$ \\
\hline & & & MTRU: & 0 & & \\
\hline * indicates Site & ecified date. & & & & $5 / 6 / 96$ & 20 of 24 \\
\hline
\end{tabular}


Tech Def \# Tech Def Name

TD Dev Time (mo)

TD Imp Time (mo)

$21 \quad$ Nitrate Removal

18

12

\begin{tabular}{|c|c|c|c|c|c|c|}
\hline TS ID & System Name & Site & \multicolumn{2}{|c|}{ Targeted Inv (m 3 ) } & TD Should start Dev. & Est. Hot Ops Date \\
\hline \multirow[t]{2}{*}{ GJ-S801C } & Mobile Evaporative Oxidation Process & GJ & MLLW: & 3.26 & $4 / 23 / 98$ & $10 / 23 / 2000$ \\
\hline & & & MTRU: & 0 & & \\
\hline \multirow[t]{2}{*}{ LA-S004 } & Mobile Plating Wastes Acids/Bases Skid & LA & MLLW: & 3.491 & $9 / 17 / 97$ & $3 / 17 / 2000$ \\
\hline & & & MTRU: & 0 & & \\
\hline \multirow[t]{2}{*}{ PO-S806 } & Lab Packs Treatment & PO & MLLW: & 30.88 & $12 / 30 / 97$ & $6 / 30 / 2000$ \\
\hline & & & MTRU: & 0 & & \\
\hline \multirow[t]{2}{*}{ PO-S807 } & Metal Recovery & PO & MLLW: & 62.25 & $3 / 30 / 99$ & $9 / 30 / 2001$ \\
\hline & & & MTRU: & 0 & & \\
\hline \multirow[t]{2}{*}{ SR-S018 } & Consolidated Incineration Facility -Liquid \&Solid Feeds & SR & MLLW: & 1379 & $6 / 30 / 94$ & $12 / 30 / 96$ \\
\hline & & & MTRU: & 0 & & \\
\hline 22 & Fission Product Removal & & \multicolumn{3}{|r|}{18} & 12 \\
\hline \multirow{3}{*}{$\begin{array}{l}\text { TS ID } \\
\text { LA-S004 }\end{array}$} & System Name & Site & \multicolumn{2}{|c|}{ Targeted Inv ( $\mathbf{m}^{3}$ ) } & TD Should start Dev. & Est. Hot Ops Date \\
\hline & Mobile Plating Wastes Acids/Bases Skid & LA & MLLW: & 3.491 & $9 / 17 / 97$ & $3 / 17 / 2000$ \\
\hline & & & MTRU: & 0 & & \\
\hline \multirow[t]{2}{*}{ OR-S005 } & TRU Processing FacilityMIPP Disposal & OR & MLLW: & 1246 & $4 / 11 / 2012$ & $10 / 11 / 2014$ \\
\hline & & & MTRU: & 1967 & & \\
\hline \multirow[t]{2}{*}{ PO-S808 } & Physical Chemical Treatment & PO & MLLW: & 77.96 & $3 / 30 / 99$ & $9 / 30 / 2001$ \\
\hline & & & MTRU: & 0 & & \\
\hline
\end{tabular}




\section{Tech Def \# Tech Def Name}

23 Internal Drum Pressure

Measurement

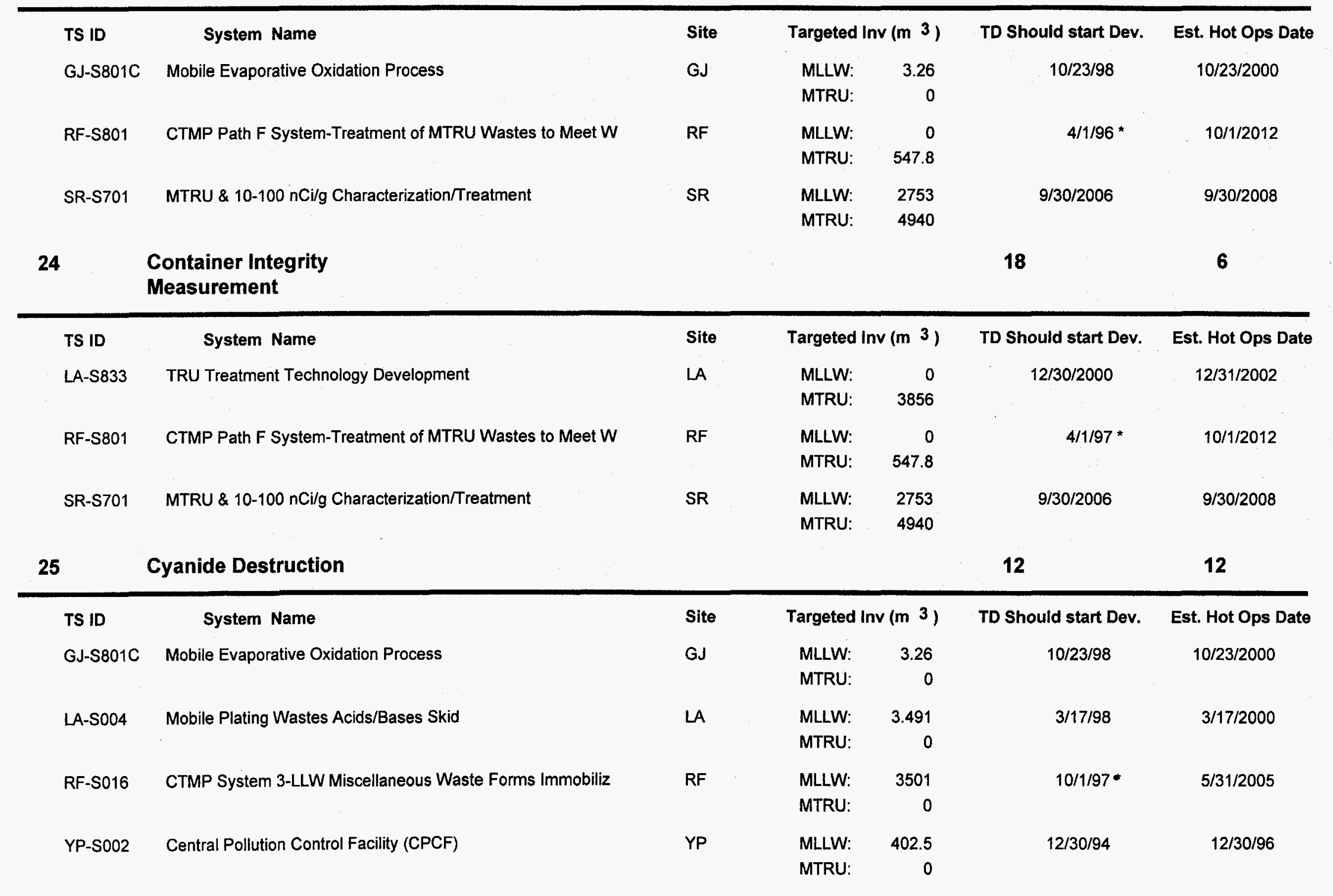

- indicates Site specified date.

TD Dev Time (mo) TD Imp Time (mo)

18

6 


\begin{tabular}{|c|c|c|c|c|c|c|}
\hline TS ID & System Name & Site & \multicolumn{2}{|c|}{ Targeted $\operatorname{lnv}\left(m^{3}\right)$} & TD Should start Dev. & Est. Hot Ops Date \\
\hline PO-S802 & Pretreatment/Regeneration & PO & MLLW: & 135.5 & 12/31/99 & $12 / 31 / 2001$ \\
\hline 27 & Evaporator Design & & & & $\mathbf{0}$ & 24 \\
\hline TS ID & System Name & Site & Targeted & $\left(m^{3}\right)$ & TD Should start Dev. & Est. Hot Ops Date \\
\hline PO-S808 & Physical Chemical Treatment & PO & MLLW: & 77.96 & 9/30/99 & 9/30/2001 \\
\hline 28 & Sludge Washing & & & & 30 & 12 \\
\hline TS ID & System Name & Site & Targeted & $\left(m^{3}\right)$ & TD Should start Dev. & Est. Hot Ops Date \\
\hline FM-S801 & Ohio Option-Mobile Chem. Tmt Project & FM & MLLW: & $\begin{array}{r}650.9 \\
0\end{array}$ & 10/30/92 & $4 / 30 / 96$ \\
\hline PO-S809 & On-Site Stabilization & PO & MLLW: & 5383 & $3 / 30 / 99$ & 9/30/2002 \\
\hline 29 & Trace Metal Removal & & & & 18 & 12 \\
\hline TS ID & System Name & Site & Targeted & $\left(m^{3}\right)$ & TD Should start Dev. & Est. Hot Ops Date \\
\hline PO-S807 & Metal Recovery & PO & $\begin{array}{l}\text { MLLW: } \\
\text { MTRU: }\end{array}$ & $\begin{array}{r}62.25 \\
0\end{array}$ & $3 / 30 / 99$ & 9/30/2001 \\
\hline PO-S808 & Physical Chemical Treatment & PO & MLLW: & 77.96 & $3 / 30 / 99$ & $9 / 30 / 2001$ \\
\hline YP-S002 & Central Pollution Control Facility (CPCF) & YP & $\begin{array}{l}\text { MLLW: } \\
\text { MTRU: }\end{array}$ & $\begin{array}{r}402.5 \\
0\end{array}$ & $6 / 30 / 94$ & $12 / 30 / 96$ \\
\hline
\end{tabular}




\begin{tabular}{|c|c|c|c|c|c|c|}
\hline $\begin{array}{l}\text { Tech Def \# } \\
30\end{array}$ & $\begin{array}{l}\text { Tech Def Name } \\
\text { Supercritical CO2 }\end{array}$ & & & TD D & $\begin{array}{c}\text { Time (mo) } \\
18\end{array}$ & $\begin{array}{l}\text { Ip Time }(\mathrm{mo}) \\
24\end{array}$ \\
\hline TS 10 & System Name & Site & Targete & $v\left(m m^{3}\right)$ & TD Should start Dev. & Est. Hot Ops Date \\
\hline FM-S801 & Ohio Option-Mobile Chem. Tmt Project & FM & $\begin{array}{l}\text { MLLW } \\
\text { MTRU }\end{array}$ & $\begin{array}{r}650.9 \\
0\end{array}$ & 10/30/92 & $4 / 30 / 96$ \\
\hline RF-S019 & CTMP System 5-LLW Surface Organic Contaminant Removal & RF & $\begin{array}{l}\text { MLLW } \\
\text { MTRU }\end{array}$ & $\begin{array}{r}2097 \\
0\end{array}$ & $4 / 1 / 97^{\bullet}$ & $4 / 1 / 2008$ \\
\hline 99 & $\begin{array}{l}\text { TD assignment being } \\
\text { assessed }\end{array}$ & & & & $\mathbf{0}$ & 0 \\
\hline TS ID & System Name & Site & Targete & $v\left(m^{3}\right)$ & TD Should start Dev. & Est. Hot Ops Date \\
\hline DP-S001 & Central Neutralization Facility & $\mathrm{DP}$ & $\begin{array}{l}\text { MLLW } \\
\text { MTRU }\end{array}$ & $\begin{array}{l}0 \\
0\end{array}$ & $12 / 1 / 96$ & $12 / 1 / 96$ \\
\hline
\end{tabular}




\section{APPENDIX A}

Listing all treatment systems from STP and disposition

A-1 


\begin{tabular}{|c|c|c|c|}
\hline TS ID & System Name & Include & Comment \\
\hline AE-S801 & Building 306, Precipitation/Filtration & No & Known process \\
\hline AE-S802 & Wet Oxidation System & No & Cannot assist due to schedule \\
\hline AE-S803 & Vitrification & No & Cannot assist due to schedule \\
\hline AE-S804 & Macroencapsulation & No & Commercially available process \\
\hline AE-S805 & Surface Decon Unit & No & No TD needs identified \\
\hline AE-S806 & Building 306, MTRU & No & Cannot assist due to schedule \\
\hline AE-S001 & Alkali Metal Passivation Booth (AMPB) & No & Cannot assist due to schedule, small scale \\
\hline AW-S037 & Sodium Process Facility & No & $\begin{array}{l}\text { Cannot assist due to schedule, known } \\
\text { process }\end{array}$ \\
\hline AW-S092 & Generator Treatment Plans at ANL-W & No & Generator treatment plan \\
\hline $\mathrm{AW}-\mathrm{S} 007$ & Remote Treatment Facility (RTF) & Yes & \\
\hline BN-S801 & Elementary Neutralization & No & Existing, known process \\
\hline $\mathrm{BN}-\mathrm{S} 802$ & Cyanide Destruction Treatability Study & No & Existing \\
\hline BN-S701 & Characterize to Determine Hazardous & Yes & \\
\hline CI-S807 & On-site Pretreatment of Sludge & No & Existing, known process \\
\hline CI-S805 & On-site Stabilization & No & Existing \\
\hline DP-S801 & Oak Ridge Commercial Option - B/C Pond & No & Commercial \\
\hline DP-S809 & Transportable Vitrification System & Yes & \\
\hline DP-S813 & Oak Ridge Commercial Disposal - B/C & No & Disposal only \\
\hline DP-S814 & Oak Ridge Commercial Treatment - Broad & Yes & \\
\hline DP-S816 & Oak Ridge Commercial Treatment - & Yes & \\
\hline DP-S819 & Commercial Disposal - TSCA Residues & No & Disposal only \\
\hline DP-S820 & Commercial Disposal - D018-D043 Debris & No & Disposal only \\
\hline DP-S002 & TSCA Incinerator & Yes & TD to enhance performance \\
\hline DP-S001 & Central Neutralization Facility & Yes & \\
\hline DS-S001 & DSSI Commercial Facility & No & Commercial \\
\hline ET-S702 & Technology Assessment & No & Small scale \\
\hline ET-S701 & Characterization Required (MLLW) & Yes & \\
\hline ET-S001 & RMDF - Treatability Study & No & Treatability study \\
\hline EV-S003 & Envirocare Commercial Facility & No & Commercial \\
\hline FM-S802 & FEMP Waste Water Treatment System & No & Existing \\
\hline FM-S805 & Thorium Nitrate Tank T-2 & No & Existing \\
\hline FM-S801 & Ohio Option-Mobile Chem. Tmt Project & Yes & Schedule Slip \\
\hline FM-S001 & UNH Treatment Facility & No & Existing \\
\hline GA-S801 & On-site Neutralization & No & Commercially available process \\
\hline GA-S701 & Characterization Required & No & No TD needs identified \\
\hline GJ-S804 & Mobile Sort/Survey/Char./Decon. & No & Existing \\
\hline GJ-S801C & Mobile Evaporative Oxidation Process & Yes & \\
\hline GJ-S801B & Mobile Thermal Desorption Process & Yes & \\
\hline $\mathrm{IN}-\mathrm{S} 005$ & WERF: Incineration - Controlled Air & Yes & \\
\hline $\mathrm{IN}-\mathrm{S} 006$ & Portable Water Treatment Unit (PWTU) & No & Existing \\
\hline IN-S013 & WROC: Sizing & No & Existing \\
\hline $\mathrm{NN}-\mathrm{S} 030$ & HEPA Filter Leaching System (HFLS) & No & Existing \\
\hline
\end{tabular}




\begin{tabular}{|c|c|c|}
\hline TS ID & System Name & Include \\
\hline
\end{tabular}

\begin{tabular}{|c|c|c|c|}
\hline IN-S033 & ICPP Debris Treat \& Containment Fac: & No & No TD needs identified \\
\hline $\mathrm{IN}-\mathrm{S} 036$ & TAN Cask Dismantlement & No & Existing \\
\hline $\mathrm{IN}-\mathrm{S} 046 \mathrm{~A}$ & MWSF: Open/Blend/Repackage Liquids and & No & No TD needs identified \\
\hline IN-S046B & MWSF: Open/Blend/Repack NonHalogenated & No & No TD needs identified \\
\hline IN-S102 & CPP659: Segregation & No & No longer planned \\
\hline IN-S104 & Lead Treatment Program: Commercial & No & Commercially available process \\
\hline IN-S128 & WROC: Mercury Retort for High Mercury & Yes & \\
\hline IN-S130 & Treatment of Waste Under 40 CFR 262.34 & No & Generator treatment plan \\
\hline IN-S132 & Generator Treatment Plans at & No & Generator treatment plan \\
\hline IN-S133 & Generator Treatment Plans at & No & Generator treatment plan \\
\hline IN-S149 & Generator Treatment Plans at & No & Generator treatment plan \\
\hline IN-S150 & Advanced Mixed Waste Treatment Project & Yes & Hot Ops Date or Contract Date \\
\hline IN-S151 & Remote Treatment Facility - RH & Yes & \\
\hline $\mathrm{IN}-\mathrm{S} 153$ & Generator Treatment Plans at & No & Generator treatment plan \\
\hline IN-S501 & SWEPP:Assay/Segregation & No & No TD needs identified \\
\hline IN-S004 & WROC/WERF: Stabilization - Portland & No & No TD needs identified \\
\hline $\mathrm{IN}-\mathrm{S} 152$ & ICPP - RH Immobilization Facility & Yes & No TD needs identified \\
\hline N-S101 & PWTU: PBF Evaporation Tank & No & No TD needs identified \\
\hline IN-S226 & WROC: Lead Recycling & No & Commercially available process \\
\hline IN-S003 & WROC: Macroencapsulation & No & No TD needs identified \\
\hline LA-S804 & Mobile Hydrothermal Processing & No & Unit canceled \\
\hline LA-S812 & Los Alamos Commercial Stabilization or & No & No TD needs identified \\
\hline LA-S833 & TRU Treatment Technology Development & Yes & \\
\hline LA-S801 & Mobile Gas Scrubbing Skid & Yes & \\
\hline LA-S701 & Further Characterization & Yes & \\
\hline LA-S004 & Mobile Plating Wastes Acids/Bases Skid & Yes & \\
\hline LA-S003 & Mobile Reactive Metals Skid & Yes & \\
\hline LA-S001 & Mobile Lead Decontamination Trailer & No & Commercially available process \\
\hline LB-S702 & Technology Assessment & No & Schedule, no defined technology \\
\hline LB-S701 & Characterization Required & No & Cannot assist due to schedule \\
\hline LB-S004 & Building 75: Unit $131 \mathrm{AFH}$ & No & Existing \\
\hline LL-S803 & Treat. Tech. Demonstrated - Mixed & No & Treatability study \\
\hline LL-S801 & On-Site Small-Scale Treatment & No & Treatability study \\
\hline LL-S701 & Characterization Required & Yes & \\
\hline LL-S702 & Technology Assessment & No & No TD needs identified \\
\hline LL-S004 & Area 514 Waste Water Treatment Tank & No & Existing \\
\hline LL-S002 & Building 513 Solidification Unit & Yes & \\
\hline NS-S003 & NSSI Commercial Facility - & No & No TD needs identified \\
\hline NT-S999 & Already Meets LDR No & No & TD needs identified \\
\hline NT-S802 & Mixed Waste Treatment Facility (MWTF) & No & Commercial \\
\hline NT-S703 & Treatability Assessment & Yes & \\
\hline NT-S702 & Evaluate Regulatory Status & No & No TD needs identified \\
\hline NT-S803 & RFP for Stabilization & No & No TD needs identified \\
\hline
\end{tabular}




\begin{tabular}{|c|c|c|c|}
\hline TS ID & System Name & Include & Comment \\
\hline NT-S701 & Characterize to Determine $\mathrm{Rad} / \mathrm{Haz}$ & Yes & Small scale \\
\hline $\mathrm{OH}-\mathrm{S} 001$ & Ohio Option - Stabilization Project & No & No TD needs identified \\
\hline OR-S805 & Oak Ridge Commercial Treatment - & No & No TD needs identified \\
\hline OR-S804 & Compressed Gases & No & No TD needs identified \\
\hline OR-S803 & ORNL Chemical Detonation Facility & No & No TD needs identified \\
\hline OR-S802 & Actions Driven By CERCLA Record of & Yes & \\
\hline OR-S005 & TRU Processing Facility/WIPP Disposal & Yes & Hot Ops Date or Earlier \\
\hline PA-S001 & C-400-D Lime Precipitation Unit & No & Existing \\
\hline PA-S701 & Meets LDR Standard and/or Needs & Yes & \\
\hline PA-S902 & Commercial Treatment and Disposal - & Yes & \\
\hline PA-S903 & Show no rad contam, then & No & No TD needs identified \\
\hline PA-S904 & Commercial Recycling & No & Commercial \\
\hline PI-S801 & Mobile Amalgamation Process (Bench & Yes & \\
\hline PO-S805 & Decontaminating Containers & No & No TD needs identified \\
\hline PO-S806 & Lab Packs Treatment & Yes & Ship date for commercial \\
\hline PO-S807 & Metal Recovery & Yes & Hot Ops Date or later \\
\hline PO-S808 & Physical Chemical Treatment & Yes & Hot Ops Date or later \\
\hline PO-S809 & On-Site Stabilization & Yes & Would like to include under DP-S814 \\
\hline PO-S901 & Commercial Offsite Recycling & Yes & Commercial \\
\hline PO-S902 & Commercial Offsite Incinerator & Yes & Commercial \\
\hline PO-S804 & Deactivate Reactive Metals & No & $\begin{array}{l}\text { Treatment will be completed by vendor i } \\
\text { FY } 96\end{array}$ \\
\hline PO-S803 & Repackage & No & $\begin{array}{l}\text { Activity will be complete by end of May } \\
96\end{array}$ \\
\hline $\mathrm{PO}-\mathrm{S} 003$ & X-622 Groundwater Treatment Facility & No & Existing \\
\hline PO-S005 & X-705 Decontamination Facility: & No & No TD needs identified \\
\hline PO-S701 & Characterization of Highly Enriched & No & Characterization activity is complete \\
\hline PO-S802 & Pretreatment/Regeneration & Yes & Commercial \\
\hline PX-S803 & Mobile Macroencapsulation & No & No TD needs identified \\
\hline PX-S004 & Burning Ground: One Cage, One Tray, & No & Unit no longer used \\
\hline PX-S801 & Mobile Stabilization Treatment Process & No & No TD needs identified \\
\hline QX-S004 & Quadrex Commercial Disposal Facility & No & Commercial \\
\hline RF-S028 & CTMP System 6-LLW Pondcrete Remix & Yes & \\
\hline RF-S801 & CTMP Path F System-Treatment of MTRU & Yes & \\
\hline RF-S020 & Residue Stabilization & Yes & \\
\hline RF-S019 & CTMP System 5-LLW Surface Organic & Yes & \\
\hline RF-S017 & CTMP System 2/4B-LLW Buildings 374/774 & Yes & \\
\hline RF-S016 & CTMP System 3-LLW Miscellaneous Waste & Yes & \\
\hline RF-S013 & Misc Aqueous Waste Handling \& & No & Existing \\
\hline RF-S004 & Process Waste Treatment Facility: & No & No TD needs identified \\
\hline RF-S001 & Building 774 - Aqueous Process Waste & No & No TD needs identified \\
\hline RL-S803 & Commercial Thermal Treatment Facility & No & Commercial contract in place \\
\hline RL-S802 & Amalgamation at Purex & Yes & No Hot Ops date available \\
\hline
\end{tabular}




\begin{tabular}{|c|c|c|c|}
\hline TS ID & System Name & Include & Comment \\
\hline
\end{tabular}

RL-S801 Encapsulation at Purex

RL-S701 Evaluating Treatment Options

RL-S007 Waste Receiving and Processing

RL-S006 Waste Receiving and Processing

SA-S813 TRU Treatment Technology Development

SA-S812 Sorting of Hazardous Debris

SA-S811 Radionuclide Separation

SA-S810 Mobile Packed Bed Reactor/Silent

SA-S807 Mobile Treatability Study:

SA-S806 TS: Chemically Deactivate Reactive

SA-S805 Treatability Study: Deactivate Water

SA-S804 Treatability Study: Chemical

SE-S005 Scientific Ecology Group (SEG)

SR-S701 MTRU \& 10-100 nCi/g

SR-S801 Waste Already Meets Treatment Standard

SR-S802 ITP \& LW Treatment - Treatability

SR-S803 Treatment of Lab Waste as a 90-Day

SR-S804 Offsite Vendor Treatment

SR-S806 D-Area Heavy Water Reclamation Process

SR-S808 Macroencapsulation as a 90-Day

SR-S809 RH Macroencaps-Containment Bldg. by

SR-S810 Macroencapsulation in a Permitted

SR-S811 Further characterization/studies

SR-S019 Processes Requiring Further Evaluation

SR-S018 Consolidated Incineration Facility

SR-S015 M-Area Vendor Treatment Process

SR-S007 Treatability Variance

SR-S004 SRL (SRTC) Ion Exchange Treatment

SR-S003 SRL (SRTC) Ion Exchange Treatment

SR-S002 F/H Area Effluent Treatment Facility

WP-S001 WIPP Option

WS-S808 Macroencapsulation with CSS

WS-S804 Amalgamation

WS-S807 Decontamination/Stabilization

WS-S803 Deactivation Followed by Stabilization

WS-S001 Site Water Treatment Plant Train 1

WS-S004 Chemical Stabilization/Solidification

WV-S803 Interim Waste Storage Facility

WV-S802 Contact Size Reduction Facility (CSRF)

WV-S702 Further Characterization Required to

WV-S001 Integrated Radioactive Waste Treatment

WV-S701 Further Characterization Required to

YP-S003 Cyanide Treatment Facility, Building

$\begin{array}{ll}\text { No } & \text { No TD needs identified } \\ \text { No } & \text { Commercial } \\ \text { No } & \text { Commercial } \\ \text { No } & \text { No TD needs identified } \\ \text { Yes } & \\ \text { No } & \text { Existing } \\ \text { No } & \text { No TD needs identified } \\ \text { Yes } & \\ \text { No } & \text { Treatability study } \\ \text { No } & \text { Treatability study } \\ \text { No } & \text { Treatability study } \\ \text { No } & \text { Treatability study } \\ \text { No } & \text { Commercial } \\ \text { Yes } & \\ \text { No } & \text { No treatment required } \\ \text { No } & \text { Existing } \\ \text { No } & \text { Treatability study } \\ \text { Yes } & \\ \text { No } & \text { Existing } \\ \text { No } & \text { Generator treatment plan } \\ \text { Yes } & \\ \text { No } & \text { No TD needs identified } \\ \text { No } & \text { No TD needs identified } \\ \text { No } & \text { No TD needs identified } \\ \text { Yes } & \\ \text { Yes } & \text { Existing } \\ \text { No } & \text { No TD needs identified } \\ \text { No } & \text { Existing } \\ \text { No } & \text { Existing } \\ \text { No } & \text { Existing } \\ \text { Yes } & \\ \text { No } & \text { No TD needs identified } \\ \text { Yes } & \\ \text { No } & \text { Existing } \\ \text { No } & \text { Cannot assist due to schedule } \\ \text { No } & \text { Existing } \\ \text { No } & \text { Cannot assist due to schedule } \\ \text { No } & \text { No TD needs identified } \\ \text { No } & \text { No TD needs identified } \\ \text { Yes } & \\ \text { No } & \text { Existing } \\ \text { Yes } & \\ \text { No } & \text { Small scale } \\ & \\ \text { No }\end{array}$




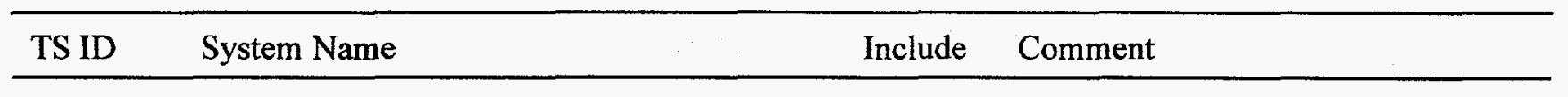

YP-S002 Central Pollution Control Facility

YP-S001 Biodenitrification Unit, Building 9818

YP-S010 Mercury Treatment Facility/CPCF

YP-S801 Actions Driven by CERCLA Record of
Yes TD enhancements to treat new wastes

No No TD needs identified

No Included as part of YP-S002

No No TD needs identified 
APPENDIX B

List of Site Codes and Names

B-1 


$\begin{array}{ll}\text { AE } & \text { Argonne National Laboratory - East } \\ \text { AW } & \text { Argonne National Laboratory - West } \\ \text { BN } & \text { Brookhaven National Laboratory } \\ \text { CI } & \text { Colonie Interim Storage Site } \\ \text { DP } & \text { K-25 Site } \\ \text { DS } & \text { DSSI Commercial Facility } \\ \text { ET } & \text { Energy Technology Engineering Center - SSFL } \\ \text { EV } & \text { Envirocare Commercial Facility } \\ \text { FM } & \text { Fernald Environmental Management Project } \\ \text { GA } & \text { General Atomics } \\ \text { GJ } & \text { Grand Junction Projects Office } \\ \text { IN } & \text { Idaho National Engineering Laboratory } \\ \text { LA } & \text { Los Alamos National Laboratory } \\ \text { LB } & \text { Lawrence Berkeley Laboratory } \\ \text { LL } & \text { Lawrence Livermore National Laboratory } \\ \text { NS } & \text { Newport News Naval Shipyard } \\ \text { NT } & \text { Nevada Test Site } \\ \text { OR } & \text { Oak Ridge National Laboratory } \\ \text { PA } & \text { Paducah Gaseous Diffusion Plant } \\ \text { PI } & \text { Pinellas Plant } \\ \text { PO } & \text { Portsmouth Gaseous Diffusion Plant } \\ \text { PX } & \text { Pantex Plant } \\ \text { QX } & \text { Quadrex Commercial Disposal Facility } \\ \text { RF } & \text { Rocky Flats Environmental Technology Site } \\ \text { RL } & \text { Richland Site } \\ \text { SA } & \text { Sandia National Laboratory - Albuquerque } \\ \text { SE } & \text { Solar Energy Research Institute } \\ \text { SR } & \text { Savannah River Site } \\ \text { WP } & \text { Waste Isolation Pilot Project } \\ \text { WS } & \text { Weldon Spring Site } \\ \text { WV } & \text { West Valley Demonstration Plant } \\ \text { YP } & \text { Y-12 Plant } \\ & \\ & \end{array}$


APPENDIX C

Prioritized Listing of Technology Deficiencies

C-1 
Mixed Waste Technology Deficiencies

Technology deficiency

Description

1. Mercury stabilization

2. Mercury amalgamation

3. NDE/NDA-initial characterization
Toxic metal contaminants regulated under the Resource Conservation and Recovery Act (RCRA) contained in mixed wastes require removal or stabilization to control solubility under the conditions of the Toxic Characteristic Leach Procedure (TCLP) before the wastes can be disposed. Under RCRA, the "low mercury" contamination level, less than $260 \mathrm{ppm}$ ( $>260 \mathrm{ppm}$ requires retorting) requires stabilization to control mercury solubility to the Universal Treatment Standards $(\leq 0.2 \mathrm{ppm})$. Verification of treatment, i.e. penetrating the entire matrix and stabilizing essentially all of the mercury in the system, is required.

Elemental mercury may be derived as a product of retorting high mercury ( $>260 \mathrm{ppm}$ ) wastes, or recovered from the off gas of a thermal treatment system, in addition to the elemental mercury streams in the DOE mixed waste inventory. Radioactive mercury can probably not be completely purified and verified for recycle. Disposal of the mercury will require amalgamation to form a stable, insoluble product for disposal. Methods and equipment designs are required for amalgamating bulk non-recyclable mercury.

Nondestructive examination (NDE) and nondestructive assay (NDA) techniques and equipment are required for the initial characterization of many hazardous wastes and all other (tramp) materials in waste drums and boxes.

Obtaining representative samples of these heterogeneous wastes and materials is made much more difficult in a radioactive environment, which escalates costs dramatically. It is necessary to determine the nature of the waste matrix in any package, confirm the presence and concentration of RCRA regulated materials and radionuclides, and identify characteristics of concern for operational safety and process continuity. This is to be accomplished non invasively or with minimal penetration. 
Mixed Waste Technology Deficiencies

Technology deficiency

Description

4. Mercury separation/ removal

5. Material handling

6. Sorting/segregation
The presences of mercury complicates the design of off gas systems, stabilization of residuals, and monitoring of all effluents. It may be advantageous to remove the mercury as a pretreatment to simplify downstream operations. New techniques must be developed to physically or chemically remove the mercury for separate stabilization. Waste matrices from which mercury separation may be required include soil, all types of process residues or sludges and particulate materials, and debris. Processing methods must ensure adequate removal, and include measuring and monitoring methods to control and verify the process.

Handling of DOE mixed waste must be accomplished safely in a radioactive environment, including containment for alpha contamination. Radioactive material containment limits access to the containers or wastes, and creates difficulties for opening of containers, removal of wastes, sorting, conditioning (feed preparation), and size reduction for feed to treatment. Many common industrial practices involving manual sorting, shredding, and conveying materials into and out of the process may not be possible due to the radioactive contamination of these wastes. Methods and equipment designs are required that will provide for handling all types of DOE waste materials in all process steps without undue risk of exposure of operating personnel to radioactivity.

Mixed waste packages contain waste materials with many different matrices. It will often be necessary to sort or segregate materials such as debris from sludges, or different types of debris matrices, before the waste can be assigned to appropriate treatment. Efficient separation of nonradioactive, or radioactive only (no RCRA regulated constituents) from mixed wastes could produce substantial savings. Because these wastes are radioactive and may contain volatile hazardous materials, the sorting and segregation must be done with adequate containment to control potential releases. 
Mixed Waste Technology Deficiencies

Technology deficiency

Description

7. Salt stabilization

8. Ash stabilization

9. Mercury monitoring
Some existing mixed wastes, and various types of waste residues that will be produced in treatment of other mixed wastes, will be high in salt content, typically chlorides, nitrates, and hydroxide sludges. These salts can be very difficult and expensive to stabilize in glass or standard Portland cement-based grouts. Polymeric compounds may contain higher concentrations of some salts in the near term, but do not chemically bind the contaminants, and an organic matrix may be undesirable for some disposal scenarios. Stabilization processes are required that increase waste loadings, improve durability and/or reduce the volume increase typical of today's standard practices. Significantly improved matrices such as new grouts based on innovative chemistries, polymers, etc. will be required.

Ash is routinely produced at DOE facilities incinerating mixed wastes. The Universal Treatment Standards have made leach resistance requirements more stringent for some heavy metals. Stabilization processes are required for this ash that increase waste loadings, improve durability and/or reduce the volume increase typical of today's standard practices. Significantly improved matrices such as new grouts based on innovative chemistries, polymers, etc. will be required.

Mercury is present in a wide variety of mixed waste matrices and at widely varying concentrations. Monitoring methods for mercury vapors are required to ensure that mercury is not being released, particularly from processes operating at elevated temperatures. Though mercury monitors are commercially available, it would be advantageous to develop real-time monitors requiring minimal consumables and low maintenance, with operating ranges covering the emission limits typical of incinerators. 
Mixed Waste Technology Deficiencies

Technology deficiency

Description

10. Alpha monitoring

11. VOC monitoring

12. Heavy metal monitoring

Processing of mixed wastes will require monitoring of wastes at all stages of handling to ensure that radioactivity, especially alpha radionuclides, are not being released, particularly with processes operating at elevated temperatures. Though alpha monitors are commercially available, it would be advantageous to develop real-time monitors requiring minimal consumables and low maintenance, with operating ranges covering the emission limits typical of alpha material processing facilities.

RCRA regulated volatile organic compounds (VOCs) are present in many mixed wastes. Because these contaminants are volatile, they are highly mobile, and will be released from newly opened packages and at any stage in processing that involves elevated temperatures. Monitoring of VOCs in the treatment facility effluent is necessary to ensure the facility is operating in accordance with environmental protection requirements. It would be advantageous to develop real-time monitors requiring minimal consumables and low maintenance, which can identify specific contaminants in operating ranges covering the emission limits typical of hazardous waste treatment facilities.

RCRA regulated heavy metals are present in many mixed wastes. In high temperature processes some of these metals can be volatilized. Though most of these metals are readily captured, some may be carried through the off gas system as fine particles, or potentially as vapors as described above for mercury. It would be advantageous to develop real-time monitors requiring minimal consumables and low maintenance, which can identify specific metals in operating ranges covering the emission limits typical of hazardous waste incinerators. 
Mixed Waste Technology Deficiencies

Technology deficiency

Description

13. Radionuclide

distribution/partitioning

14. Waste form performance

15. HEPA filter improvements
During mixed waste treatment processes, particularly thermal systems, the distribution and migration of radionuclides throughout the processing system must be understood to ensure adequate control. Only limited data exist to predict the fractional distribution of radionuclides between the off gas, the final waste form, and any secondary waste streams. More complete information on the radionuclide distribution in high temperature mixed waste treatment processes is needed to support equipment design and process permitting.

The performance criteria for regulated hazardous constituents are generally established in state and EPA regulations, such as TCLP requirements. No such requirements have been finalized for radionuclides, so the increased durability of vitrified, slagged, or encapsulated waste forms has not been incorporated into disposal facility permitting or performance assessments. An objective, technically defensible evaluation of the long-term performance of advance waste forms must be conducted. The evaluation must satisfy regulator and stakeholder concerns to allow flexibility in siting and operating lowlevel waste disposal facilities to best exploit the more durable, higher waste-loading forms.

High-efficiency particulate air (HEPA) filters are a vital part of the environmental protection systems for any facility processing radioactive materials. Discarding these filters contributes significantly to the net waste produced from waste treatment operations. The filters are also a potential point for accidental breakthrough and release of radionuclides. A stronger, high-temperature, longer lived HEPA filter, that can survive greater pressure drop, and that requires less frequent replacement, or that can be cleaned and reused, is needed. New filter designs should be designed to replace standard filters with minimal retrofit of existing systems. 
16. Mercury filter

17. Molten product - decanting

18. Comparative

analysis/aqueous
Mercury is present in a wide variety of mixed waste matrices and at widely varying concentrations. Mercury will be present in some wastes for which mercury treatment will not be indicated. At elevated temperatures in many waste treatment processes mercury will vaporize.

Therefore, the off gas system for most, if not all, mixed waste treatment systems will require a step for mercury removal. Typically this step is a water quench, which condenses most volatiles, but creates a mixture in the blowdown stream that is difficult to treat. A potential enhancement to this traditional design would be a selective mercury removal step, which removes essentially all of the mercury from the off gas stream for separate treatment. Candidate processes exist, but have not been demonstrated on mixed waste off gas streams.

Many treatment processes being developed for mixed wastes will operate with molten material. These processes require the transfer of a molten product from the furnace to another vessel for final handling/disposal. Usually separation of the molten material from untreated waste and perhaps a slag or metal phase is required. Operating techniques and equipment design are required to accomplish this decanting or transfer of molten phases in an effective, reliable, and safe manner applicable to a radioactive environment.

A comparative analysis on the efficacy, reliability, applicability, and maintainability of the many processes now being developed for destruction of organic contamination in wastewater containing radionuclides must be done to select two or three processes to be developed in Item 19 below. 
Mixed Waste Technology Deficiencies

Technology deficiency

19. Aqueous organic nonthermal destruction

20. Refractory performance

21. Nitrate removal

22. Fission product removal
Description

Successful destruction/removal of regulated organic constituents in wastewater is necessary for many aqueous plant recycle streams, as well as mixed waste solutions currently being stored in the DOE inventory. Organic containing aqueous streams in treatment processes may contain dissolved and suspended solids along with any of a wide variety of organics. Of particular concern are halogenated and high molecular weight compounds. Several candidate technologies exist, but it remains to be demonstrated that any one or a combination can reliably treat all of the organic constituents expected to be present in mixed waste and be operated in a radioactive environment.

Refractory lifetime is a limiting factor for operation of incinerators and most other thermal treatment processes. Refractories are degraded by acid and caustic conditions and thermal cycling, and sorb metals and radionuclides during operation. Replacement of refractories decreases operating time, adds to operating costs and personnel exposure, and generates a secondary mixed waste stream. Improved refractories, or operating techniques better suited to the DOE-specific processing conditions, are required for long-term processing success.

Most DOE chemical operations are conducted in nitrate based systems because of process simplicity and material compatibility. As a result, many of DOE mixed wastes contain nitrates. Nitrates are reactive oxidizers, which can cause complications in treatment processes and additional difficulties in waste stabilization processes. Methods are needed to destroy or remove residual nitrates from sludges and wastewaters.

Some mixed wastes contain elevated levels of radionuclides requiring special handling and treatment. Techniques for selective removal of nuclides such as cesium, strontium, and tritium would simplify processing of the balance of the matrix. Methods are needed for removal or significant reduction of the concentrations of fission products from mixed waste, especially process residues and sludges. 
Mixed Waste Technology Deficiencies

Technology deficiency

Description

23. Internal drum pressure measurement

\section{Container integrity} measurement

25. Cyanide destruction

26. Thermal desorption

27. Evaporator design
The occurrence of internal drum pressure is a hazard to operating personnel and to process equipment. Methods are needed to measure internal drum pressure without penetrating the drums.

Many DOE wastes have been stored for a significant time period. Some of the waste containers have suffered measurable degradation. Containers at or near failure due to stress and corrosion are a potential hazard to personnel and operating systems. Methods are needed to test the integrity of stored containers to identify any that may require particularly careful handling or overpack in preparation for managing or processing of the contents.

Cyanide occurs in DOE wastes primarily from metal cleaning and processing operations. RCRA regulations require the destruction of cyanide anion when it is present. This is normally done in a simple one step aqueous oxidation reaction. Not all DOE wastes containing cyanides are amenable to treatment by this aqueous based reaction. Methods are required to treat cyanide in the presence of interfering dissolved, suspended, and matrix materials.

Thermal desorption of organic hazardous constituents and mercury from process residues, sludges, soils, and debris has been commercialized, and is now proposed for a number of mixed waste streams. Many of these wastes are physically and chemically complex materials. Methods are required to minimize pretreatment to adequately prepare the wastes so that the contaminants can escape, to provide thermal processing such that cleanup levels can be attained while maintaining radionuclide containment, and to verify LDR compliance.

Processes for treatment of mixed wastes will generate many aqueous streams for recycle within the plant. Recycling and final cleaning of water for discharge usually involves one or more evaporation steps. Evaporators are subject to fouling and corrosion and use fairly large quantities of energy. Better designs are needed for evaporators for DOE wastespecific treatment plant streams. 
28. Sludge washing

29. Trace metal removal

30. Supercritical $\mathrm{CO}_{2}$
Sludge washing may be a key step in nonthermal treatment processes. This treatment is also potentially applicable to small quantities of waste. Organic removal requirements in RCRA regulations are generally well beyond that which has been demonstrated for any of the candidate processes.

Alternative approaches are required, or methods are needed to enhance the performance of the candidate sludge washing technologies, to demonstrate feed preparation and washing of process residues, sludges, and particulates to RCRA requirements.

Some wastewater treatment facilities in the DOE complex are subject to new permitting requirements which mandate extremely low-levels for some metals in effluents (e.g., $0.001 \mathrm{mg} / \mathrm{L}$ cadmium, $0.003 \mathrm{mg} / \mathrm{L}$ lead, and $0.004 \mathrm{mg} / \mathrm{L}$ silver). Standard water polishing ion-exchange resins are not sufficiently specific to avoid depletion by other ions in the wastes. Other techniques are needed to meet permit requirements while minimizing secondary waste generation.

Supercritical $\mathrm{CO}_{2}$ extraction similar to that commercially practiced for a variety of standard applications has been proposed for treatment of a number of mixed waste streams in lieu of thermal processing. Many of these wastes are physically and chemically complex materials. Methods are needed to enhance the effectiveness of supercritical $\mathrm{CO}_{2}$ for removal of organics from process residues, sludges and particulates, soils, and debris (especially more complex pieces of debris). Techniques to minimize pretreatment to adequately prepare the wastes so that the organics can be removed, and the wastes can be fed and removed from the supercritical environment while maintaining radionuclide containment are required. Compliance with LDR must also be verified. 
APPENDIX D

Mortgaged Activities

D-1 
Mortgaged Activities

Mortgaged Activities Description

Controlled Emissions

Demo

Delphi Detox

Direct Chemical

Oxidation

Macroencapsulation

at Envirocare

of Utah, Inc.

PHP

RCRA NDA

Characterization
Western Environmental Technologies Office (WETO) located in Butte, Montana, is currently engaged in an extensive testing program to significantly reduce air, fluid, and solid emissions from DOE thermal systems. This program is targeted at reducing air emissions to standards set in EPA's proposed combustion rule. Testing of Continuous Emissions Monitors (CEMs) to verify if and how much of a particular substance is being emitted continues.

Delphi is a small business that has developed a proprietary process for the destruction of organic wastes. This process uses a solution of ferric chloride, hydrochloric acid, water, and small amounts of other constituents to oxidize organic wastes. This technology will be demonstrated on surrogate wastes at Savannah River using a full scale treatment unit. Pending site approval, the unit will be moved to Rocky Flats for further waste testing and treatment.

Direct Chemical Oxidation is a technology being developed at Lawrence Livermore National Laboratories (LLNL). The process is simple, using peroxydisulfide as an oxidant to destroy organic liquids and solids. After oxidation, the sulfide solution is recycled by electrolysis. The process operates at low temperature and pressure, and produces no severe corrosion by-products. A bench scale test is planned this year followed by pilot scale testing in FY 1997.

In FY 1997, Envirocare will complete treatment and disposal of the remainder of the 500,000 lbs capacity under the Cooperative Agreement with DOE-ID. This Cooperative Agreement procures polymer macroencapsulation of 500,000 lbs of mixed waste lead and debris, followed by disposal in the RCRA Subtitle C landfill at Envirocare. Waste streams from approximately 24 sites will be treated and disposed by Envirocare through the Cooperative Agreement. While treating these waste streams, Envirocare will scale its process up from 5 gal units to B-12 bins. This scale-up, which will complete this commercial feasibility study, will be completed in FY 1997.

The PHP activity will be used to demonstrate the treatment of mixed wastes using a plasma technology. This thermal approach for mixed waste treatment employs commercially-available technology from the nonradioactive specialty metals processing industry. The immediate challenge of this work is modification of the technology to accommodate the radioactive species in mixed waste. The resulting data and process system may be used by other facilities within the complex.

The purpose of this activity is to evaluate commercial noninvasive characterization technologies for RCRA-regulated metals and to make recommendations to the MWFA on any additional development needs required in this area. Performance 
Mortgaged Activities

Mortgaged Activities

Description

testing of the technologies will be conducted on both single blind surrogates and on inorganic sludge surrogates containing $\mathrm{Cd}, \mathrm{Hg}$, and $\mathrm{Pb}$. This mortgaged activity addresses part of Technology Deficiency \#12-Heavy Metal Monitoring.

Russian Denox

Torch Life Extension
Scientists at the Boreskov Institute of Catalysis in Russia are working on a new catalyst to be used in off gas systems to improve NOx destruction efficiency, reduce ammonia slip, cost less than currently used catalysts, and to be disposable in non-RCRA landfills. DOE personnel at Lawrence Livermore National Laboratories and Western Environmental Technologies Office (WETO) are working with the Russians to develop and test the new catalysts.

The purpose of this activity is to evaluate different torch concepts and models from four different manufacturers to help DOE focus on the most reliable type of torch for use in the mixed waste treatment systems. Work will be conducted with the torch manufacturers support, to increase electrode life and torch reliability. This activity was identified as a need in the DOE complex, although it was not one of the thirty prioritized deficiencies listed in the technical baseline, since a plasma process has not yet been identified as a primary treatment technology at any facility.

The Transportable Vitrification System, developed at Savannah River, and to be demonstrated on $80,000 \mathrm{~kg}$ of actual mixed waste sludge at Oak Ridge, consists of a joule-heated melter and associated equipment designed on skids for mobility. The original (first driving objective) for TVS was to demonstrate vitrification of actual mixed waste. As a consequence, operations of TVS will add data for correcting MWFA deficiencies addressing refractory performance, and molten product decanting. In contrast, the flexibility for TVS to take a wider range of waste feeds could be greatly increased by correcting the deficiencies addressing CEM (alpha, mercury, VOC, and heavy metals monitoring), mercury removal and stabilization. 